\title{
Bases aditivas de um ponto de vista topológico e prime gaps de um ponto de vista algébrico
}

Luan Alberto Ferreira

TESE APRESENTADA

$\mathrm{AO}$

Instituto DE Matemática e EstatísticA

DA

Universidade de SÃo Paulo

PARA

OBTENÇÃ̃ DO TÍTULO

$\mathrm{DE}$

Doutor EM CIÊNCIAS

Programa: Pós-Graduação em Matemática do IME-USP

Orientador: Prof. Dr. Hugo Luiz Mariano

Coorientador: Prof. Dr. Paulo Agozzini Martin

Durante o desenvolvimento deste trabalho o autor recebeu auxílio financeiro da CAPES

São Paulo, setembro de 2016 


\section{Bases aditivas de um ponto de vista topológico e prime gaps de um ponto de vista algébrico}

Esta tese contém as correções e alterações sugeridas pela Comissão Julgadora durante a defesa realizada por Luan Alberto Ferreira em 28/07/2016.

O original encontra-se disponível no Instituto de Matemática e Estatística da Universidade de São Paulo.

Comissão Julgadora:

- Prof. Dr. Hugo Luiz Mariano (orientador) - IME-USP

- Prof ${ }^{a}$. Dra ${ }^{\mathrm{a}}$. Ires Dias - ICMC-USP

- Prof. Dr. Oziride Manzoli Neto - ICMC-USP

- Prof. Dr. Marcelo Escudeiro Hernandes - UEM

- Prof. Dr. José Plínio de Oliveira Santos - UNICAMP 


\section{Agradecimentos}

Mais uma vez chega o difícil momento de agradecer a quem me acompanhou, direta ou indiretamente, na produção desta tese. Peço desculpas desde já aqueles que julguei mal, seja por excesso, seja por falta. Não quero cometer injustiças.

Agradeço inicialmente aos meus colegas de São Carlos Carlos Henrique Assunção, Henrique Caldara, Marcelo Moreno e Thales Noel Pereira Pallu, para com os quais me desculpo pela ausência de agradecimentos a vocês na minha dissertação de mestrado. Devo uma ótima cerveja para cada um por isso!

Agradeço à Wiviane Valério por tudo que vivemos e aprendemos juntos e que, por imaturidade minha, deixei de agradecê-la em minha dissertação de mestrado.

Agradeço aos meus colegas do IME por termos conseguido criar um ambiente incrivelmente agradável para se trabalhar. Em especial, agradeço a Adam Petzet Rudnik, Adilson Konrad, Ana Cláudia da Silva Moreira, Ariadne Nogueira, Bartira Maués, Belmiro Galo da Silva, Benigno Oliveira Alves, Bruna Cassol dos Santos, Daniel de Brito Reis, Danielle dos Santos Mingatos, Darllan Conceição Pinto, Dimi Rocha Rangel, Dúlio Ferreira Santos, Fernanda Evangelista Ferreira da Silva, Gilberto Luiz Angelice Camargo, Gilberto Pereira Sassi, Glauce Barbosa Verão, Guilherme Galina Loch, Helton Graziadei de Carvalho, Hugo Rafael Oliveira Ribeiro, Jean Carlos Nakasato, Jean Cerqueira Berni, João Paulo Cirineu de Jesus, Joelson da Cruz Campos, Josefa Itailma da Rocha, Leandro Antunes, Maikel Antônio Samuays, Maurício Zahn, Michael Alexander Rincon Villamizar, Renato Vasconcellos Vieira, Robson Aparecido Figueiredo, Rodrigo Figueiredo e Vanessa Steindorf, pelos excelentes momentos que passamos juntos!

Agradeço aos meus eternos amigos do Vale do Paraíba por sempre estarem ao meu lado: Caroline Santos Rodrigues da Silva, Denise Clarice Caputo de Souza, Fernando Luiz Bustamante Bueno Oliveira, Gabriel O. Godoy, Gustavo Henrique Prevatto Zani, Lucas Renan Coelho Teixeira, Luis Fernando da Costa Oliveira, Luiz Rafael dos Santos Leite, Maria Clara Alvarenga, Miriam Silva Freitas Dias, Nilson Henrique Chagas Oliveira, Orlando Pasqual Filho (é do Vale sim, rs), Paula Salles Gória, Priscila Aparecida Gonçalves, Suzana Lobato e Thiago Augusto dos Santos Silva. Tenho um carinho muito grande por cada um de vocês!

Agradeço aos seguintes professores e professoras do IME que tive o prazer de conhecer e de, em alguns casos, poder trabalhar junto enquanto aluno: Alexandre Lymberopoulos, Ana Paula Jahn, Clodoaldo Grotta Ragazzo, Daciberg Lima Gonçalves, Daniel Victor Tausk, Eduardo do Nascimento Marcos, Flávio Ulhoa Co- 
elho, Henrique Guzzo Junior, Marcos Martins Alexandrino da Silva, Odilon Otávio Luciano, Paolo Piccione, Ricardo dos Santos Freire Júnior, Rosa Maria dos Santos Barreiro Chaves, Severino Toscano do Rêgo Melo e Zara Issa Abud.

Reitero meus agradecimentos aos professores que já tive e que me ajudaram a chegar até aqui. Dentre eles, agradeço especialmente aos professores e professora Ires Dias, Luiz Augusto da Costa Ladeira, Oziride Manzoli Neto e Sérgio Henrique Monari Soares por sempre acreditarem em mim, e peço que os outros (são muitos) com os quais tenho elevada estima sintam-se representados por esses.

Agradeço ao meu co-orientador e professor Paulo Agozzini Martin pela sua atenção e disponibilidade em me ajudar a construir esta tese. O precioso tempo com o qual passamos discutindo matemática foi muito importante para mim, professor.

Agradeço também ao meu orientador e professor Hugo Luiz Mariano por ter me dado a oportunidade de poder estudar o que eu queria, por todas as conversas que tivemos e por me ajudar a ampliar meus horizontes tanto matemáticos quanto profissionais. Sou imensamente grato por ter sido seu orientando. Muito obrigado, professor!

Agradeço aos funcionários do IME - USP, que sempre me trataram com atenção, cordialidade e respeito.

Agradeço a minha família que me apoiou.

Agradeço à Erika Priscila Ferreira da Silva por todos os momentos que passamos juntos, e por tudo que conversamos juntos.

Agradeço à Diana Renata Gonçalves Gama por tudo que vivemos juntos, por ter me apresentado São Paulo de uma maneira incrível e por ter me ensinado tanto sobre a vida.

Em especial, agradeço à Mariana Bueno Zanutto, por todo o suporte que você tem me dado, pelo carinho e pelos momentos lindos que vivemos juntos.

Finalmente, agradeço à Capes pelo suporte financeiro. 


\section{Resumo}

Título: Bases aditivas de um ponto de vista topológico e prime gaps de um ponto de vista algébrico.

Esta tese objetiva apresentar duas abordagens inovadoras acerca de dois assuntos clássicos da teoria dos números: bases aditivas e prime gaps.

O primeiro tópico é estudado de um ponto de vista topológico, com o intuito de oferecer uma visão abrangente e resultados gerais sobre o conjunto de todas as bases aditivas, não versando sobre uma base aditiva específica, como é de costume. Por meio da introdução de uma métrica, são apresentadas várias ferramentas topológicas que permitem tratar problemas de difícil ataque direto sobre bases aditivas por meio de argumentos indiretos sobre bases melhor comportadas e suficientemente próximas das originalmente consideradas.

Já a contribuição sobre prime gaps é realizada utilizando ferramentas algébricas, no lugar das analíticas, como habitual. Por meio de técnicas oriundas tanto da teoria de Galois quanto da teoria algébrica dos números, é apresentado um estudo da conjectura de Firoozbakht, juntamente com algumas de suas consequências, caso ela venha a ser provada.

Palavras-chave: Bases aditivas finas, prime gaps, conjectura de Firoozbakht. 


\section{Abstract}

Title: Additive bases from a topological point of view and prime gaps from an algebraic point of view.

This thesis aims to present two innovative approaches about two classical subjects on number theory: additive bases and prime gaps.

The first topic is studied from a topological point of view, in order to offer a comprehensive treatment and general results on the set of all additive bases, not dealing with one specific additive basis, as usual. By the introduction of a metric, it's presented a variety of topological tools that allows to treat problems of difficult direct attack on additive bases through indirect arguments on bases better behaved and sufficiently close to the originally considered.

The contribution on the prime gaps subject is performed by the use of algebraic tools instead of analytical methods, as usual. Utilizing techniques from Galois theory and algebraic number theory, it's presented a study of the Firoozbakht's conjecture, along with some of its consequences if the conjecture is proved.

Keywords: Thin additive bases, prime gaps, Firoozbakht's conjecture. 


\section{Sumário}

Lista de símbolos $\quad$ ix

Introdução $\quad$ xi

1 O estado-da-arte em teoria aditiva dos números 1

1.1 Resultados clássicos . . . . . . . . . . . . . . . . . . . . . . . 1

1.2 Ordem assintótica . . . . . . . . . . . . . . . . . . 3

1.3 Densidade de Schnirelmann . . . . . . . . . . . . . . . . 5

1.4 Componentes essenciais e a desigualdade de Plünnecke . . . . . . . . 6

1.5 Bases finas . . . . . . . . . . . . . . . . . . 8

1.6 Teoremas de P.A.'s . . . . . . . . . . . . . . . . . 9

1.7 Problemas em aberto . . . . . . . . . . . . . . . . . . 10

2 O espaço métrico das bases aditivas $\quad 15$

$2.1 \quad$ Métrica em $\mathcal{A} \ldots \ldots \ldots \ldots \ldots$

2.2 Primeiro pior caso e pseudocontinuidade . . . . . . . . . . . . 22

2.3 Caracterização topológica de $(\mathcal{A}, d) \ldots \ldots$. . . . . . . . . . . . 32

3 Bases aditivas finas e aplicações $\quad 35$

3.1 Um binômio de Newton para bases aditivas . . . . . . . . . . . . . . . 35

3.2 Bases aditivas finas em $(\mathcal{A}, d) \ldots \ldots$. . . . . . . . . . . 38

3.3 Funções de $\mathcal{A}$ em $\mathcal{A} \ldots \ldots$. . . . . . . . . . . . . . . . . . . . . . . . 41 
4 O problema das P.A.'s em bases aditivas 49

4.1 O problema das P.A.'s . . . . . . . . . . . . . . . . . 49

4.2 Uma aplicação do teorema de Singer . . . . . . . . . . . . . . . . 51

5 Prime gaps e a conjectura de Firoozbakht 53

5.1 O estado-da-arte em prime gaps . . . . . . . . . . . . . . . 53

5.2 A conjectura de Firoozbakht e algumas consequências . . . . . . . . . 54

5.3 Uma consequência do teorema de Zhang . . . . . . . . . . . . . . . . 59

6 Aplicações da teoria algébrica dos números aos prime gaps $\quad 61$

6.1 Background da teoria algébrica dos números . . . . . . . . . . . . 61

6.2 Estudo do número $a_{n} \ldots \ldots \ldots \ldots$. . . . . . . . . . . 64

$\begin{array}{ll}\text { Trabalhos futuros } & 69\end{array}$

$\begin{array}{ll}\text { Referências bibliográficas } & 71\end{array}$ 


\title{
Lista de símbolos
}

\author{
Símbolo Significado
}

$\mathbb{N} \quad\{z \in \mathbb{Z} ; z \geq 0\}$, conjunto dos números naturais

$\mathbb{Z} \quad$ conjunto dos números inteiros

$\mathbb{Z}_{+} \quad\{z \in \mathbb{Z} ; z>0\}$, conjunto dos inteiros positivos

$\mathbb{Q} \quad$ conjunto dos números racionais

I $\quad$ conjunto dos números irracionais

$\mathbb{R} \quad$ conjunto dos números reais

$\mathbb{C} \quad$ conjunto dos números complexos

$\mathbb{P} \quad$ conjunto dos números primos

$\mathbb{P}^{*} \quad \mathbb{P} \cup\{0,1\}$

A conjunto dos números algébricos

$\mathcal{C}$ conjunto de Cantor

$o(n, A) \quad$ ordem de $n \in \mathbb{N}$ sobre $A$

$o(A) \quad$ ordem da base aditiva $A$

$o_{\infty}(A) \quad$ ordem assintótica da base aditiva $A$

$\sigma(A) \quad$ densidade de Schnirelmann de $A$

$\operatorname{ppc}(A) \quad$ primeiro pior caso de $A$

$\mathcal{A} \quad$ conjunto das bases aditivas

$\mathcal{F} \quad$ conjunto das bases finas

$\mathcal{A}_{h} \quad\{A \in \mathcal{A} ; o(A)=h\}$

$\mathcal{A}_{h}^{m} \quad\{A \in \mathcal{A} ; o(A)=h, \operatorname{ppc}(A)=m\}$

$A^{k} \quad\left\{a^{k} ; a \in A\right\}$

$P_{m} \quad$ conjunto dos números $m$-gonais

$F_{h} \quad$ base aditiva fina de Raikov-Stöhr de ordem $h$

$R[x] \quad$ conjunto dos polinômios na variável $x$ com coeficientes no anel $R$

$A(n) \quad \#(A \cap\{1, \ldots, n\})$

$A+B \quad\{a+b ; a \in A, b \in B\}$

$A \triangle B \quad(A-B) \cup(B-A)$

$h A \quad A+\cdots+A(h$ vezes $)$

$W(k, r) \quad$ número de van der Waerden de $(k, r)$

$r(n, A)$ número de representações de $n \in \mathbb{N}$ como soma de $o(A)$ elementos de $A$

$\partial X \quad$ fronteira de $X$

$\mathcal{B}(A, r) \quad\{B \in \mathcal{A} ; d(A, B)<r\}$

$\mathcal{B}[A, r] \quad\{B \in \mathcal{A} ; d(A, B) \leq r\}$

$\mathcal{S}(A, r) \quad\{B \in \mathcal{A} ; d(A, B)=r\}$

$E \quad\{A \in \mathcal{A} ; \mathbb{N}-A$ é finito $\}$ 


\section{Símbolo Significado}

$\lfloor x\rfloor \quad$ parte inteira inferior de $x$, i.e., $\max \{z \in \mathbb{Z} ; z \leq x\}$

$\lceil x\rceil \quad$ parte inteira superior de $x$, i.e., $\min \{z \in \mathbb{Z} ; z \geq x\}$

$\langle x\rangle \quad$ parte fracionária de $x$, i.e., $x-\lfloor x\rfloor$

$\overleftarrow{x} \quad\{y \in \mathbb{N} ; 1 \leq y \leq x\}$

$A_{n} \stackrel{p}{\rightarrow} A \quad$ sequência $\left\{A_{n}\right\}_{n}$ pseudoconverge para $A$

$n ! \quad$ fatorial de $n$

$p_{n} \quad n$-ésimo número primo

$g_{n} \quad p_{n+1}-p_{n}, n$-ésimo prime gap

$\pi(x) \quad \#\{p \in \mathbb{P} ; p \leq x\}$, função contadora dos números primos

$Y_{k} \quad\left\{n \in \mathbb{Z}_{+} ; g_{n}=k\right\}$

$\mathcal{O} \quad$ conjunto dos inteiros algébricos

$\mathcal{O}_{K} \quad \mathcal{O} \cap K$, anel de inteiros do corpo de números $K$

$\xi_{n} \quad e^{2 \pi i / n}$

$\gamma \quad$ constante de Euler-Mascheroni

$\varphi(n) \quad \#\{x \in \overleftarrow{n} ; \operatorname{mdc}(x, n)=1\}$, função $\varphi$ de Euler

$f(x) \ll g(x) \quad$ existe $c>0$ tal que $|f(x)| \leq c g(x), \forall x \in \operatorname{Dom}(f)=\operatorname{Dom}(g)$

$f(n) \sim g(n) \quad \lim _{n \rightarrow \infty} \frac{f(x)}{g(x)}=1$ 


\section{Introdução}

Esta tese objetiva apresentar duas abordagens inovadoras acerca de dois assuntos clássicos da teoria dos números: bases aditivas e prime gaps. O primeiro tópico é estudado de um ponto de vista topológico, com o intuito de oferecer uma visão abrangente e resultados gerais sobre o conjunto de todas as bases aditivas, não versando sobre uma base aditiva específica, como é de costume. Este estudo é feito a partir da introdução de uma métrica no conjunto das bases aditivas, e a partir disto são obtidos uma variedade razoável de resultados sobre este conjunto. Este tópico será desenvolvido nos primeiros quatro capítulos desta tese.

O capítulo um, para a conveniência do leitor, apresenta uma visão geral da teoria aditiva dos números, com ênfase nos resultados obtidos até agora. Uma parcela razoável dos conceitos abordados e dos resultados citados serão usados com frequência nos três capítulos subsequentes. Há também uma lista de problemas em aberto nesta área, cujo intuito é mostrar ao leitor quais são alguns dos desafios ainda a serem superados.

O capítulo dois apresenta a métrica usada no conjunto das bases aditivas sobre a qual será desenvolvido este trabalho, e as principais propriedades topológicas deste espaço. Em particular, é apresentada uma caracterização topológica deste espaço métrico.

O capítulo três exibe resultados mais refinados acerca deste espaço métrico, fazendo uso das bases aditivas finas. Bases aditivas finas são bases aditivas que, em um certo sentido, têm "o mínimo possível" de elementos. É demonstrado que, ao contrário do que a intuição possa dizer, o conjunto das bases aditivas finas é denso no espaço métrico estudado. Isto tem implicações importantes para toda uma classe de funções cujo domínio e contradomínio são este espaço métrico. Em particular, o fato de que o conjunto das bases aditivas finas é denso no conjunto de todas as bases permite exibir um critério bastante específico de quando podemos acrescentar certo conjunto de números a uma determinada base aditiva sem alterar sua ordem.

O capítulo quatro apresenta o problema de encontrar progressões aritméticas no complementar de certas bases aditivas. Em particular, é mostrado que o conhecimento básico que se têm acerca desse problema é basicamente o melhor que pode ser obtido.

Já a contribuição sobre prime gaps é realizada utilizando ferramentas algébricas, no lugar das analíticas, como habitual. Isto será desenvolvido nos dois últimos capítulos desta tese. Utilizando técnicas oriundas tanto da teoria de Galois quanto 
da teoria algébrica dos números, é apresentado um estudo da conjectura de Firoozbakht, juntamente com algumas de suas consequências, caso ela venha a ser provada.

Mais especificamente, o capítulo cinco apresenta um breve resumo do estadoda-arte em prime gaps, além de apresentar o enunciado da conjectura de Firoozbakht. Para a conveniência do leitor, é apresentada uma série de implicações da conjectura de Firoozbakht. O intuito disso é mostrar a força que tem esta conjectura. Ao fim do capítulo, utilizando o recente teorema de Zhang sobre prime gaps é mostrada que a conjectura de Firoozbakht é verdadeira pelo menos um número infinito de vezes.

Por fim, o sexto capítulo desta tese apresenta um estudo algébrico da conjectura de Firoozbakht, utilizando ferramentas oriundas tanto da teoria algébrica dos números quanto da teoria de Galois. Desta forma, pretende-se utilizar maquinários algébricos ao ataque deste difícil problema. 


\section{Capítulo 1}

\section{O estado-da-arte em teoria aditiva dos números}

Este capítulo dedica-se a apresentar a maioria dos tópicos estudados em teoria aditiva dos números, assim como seus principais resultados, alguns dos quais serão livremente usados no decorrer desta tese.

\subsection{Resultados clássicos}

A teoria aditiva dos números é o ramo da teoria dos números que estuda o comportamento de certos conjuntos de números (mais comumente subconjuntos de $\mathbb{N}$ ) sob a operação de adição. O estereótipo de resultado da teoria aditiva dos números é o teorema dos quatro quadrados de Lagrange.

Teorema 1.1.1 (Lagrange, 1770). Todo número natural pode ser escrito como a soma de quatro quadrados.

A primeira demonstração que vi deste teorema foi no excelente livro [78], p. 217-223. Conjuntos como os quadrados são os principais objetos estudados na teoria aditiva dos números: as bases aditivas.

Definição 1.1.2. Sejam $\{0,1\} \subseteq A \subseteq \mathbb{N}$ e $n \in \mathbb{N}$. Definimos a ordem de $n$ sobre $A$ como sendo o menor natural o $(n, A)$ tal que $n$ pode ser escrito como a soma de $o(n, A)$ elementos de $A$.

Definição 1.1.3. Seja $\{0,1\} \subseteq A \subseteq \mathbb{N}$. Se $\max \{o(n, A) ; n \in \mathbb{N}\}<\infty$, definimos a ordem de $A$ por $o(A)=\max \{o(n, A) ; n \in \mathbb{N}\}$ e dizemos que $A$ é uma base aditiva de ordem o $(A)$. Denotamos o conjunto de todas as bases aditivas por $\mathcal{A}$.

Um dos problemas centrais da teoria aditiva dos números consiste em saber se um dado conjunto $\{0,1\} \subseteq A \subseteq \mathbb{N}$ é uma base aditiva e, em caso afirmativo, determinar sua ordem. 
Como 7 não pode ser escrito como a soma de três quadrados, segue do teorema de Lagrange que $\mathbb{N}^{2} \in \mathcal{A}$ e que $o\left(\mathbb{N}^{2}\right)=4$. É fácil ver que se $A \in \mathcal{A}$, então $A$ é infinito. Legendre completou o teorema dos quatro quadrados de Lagrange com seu famoso teorema dos três quadrados.

Teorema 1.1.4 (Legendre, 1797-1798). Um número natural pode ser escrito como a soma de três quadrados se, e somente se, ele não é da forma $4^{a}(8 b+7), a, b \in \mathbb{N}$.

Recomendamos [63], p. 134 - 139, para uma prova deste teorema. Na verdade, o teorema dos quatro quadrados de Lagrange é um caso particular de dois teoremas mais gerais em teoria aditiva dos números. O primeiro deles é conhecido como teorema dos números poligonais de Fermat. Em 1638, Fermat afirmou sem demonstrar que todo número natural pode ser escrito como a soma de três números triangulares, quatro números quadrados, cinco números pentagonais e assim por diante. O conjunto dos números $m$-gonais, $m \geq 3$, é definido por

$$
P_{m}=\left\{\frac{(m-2) n(n-1)}{2}+n ; n \in \mathbb{N}\right\} \text {. }
$$

O segundo passo para a demonstração deste teorema foi dado por Gauss.

Teorema 1.1.5 (Gauss, 1796). Todo número natural pode ser escrito como a soma de três números triangulares.

Note que o teorema dos números triangulares de Gauss é equivalente ao fato de que todo número natural congruente a 3 módulo 8 pode ser escrito como a soma de três quadrados ímpares. Uma vez que Legendre determinou quais inteiros podem ser escritos como a soma de três quadrados, podemos ver o teorema dos números triangulares de Gauss como um corolário do teorema dos três quadrados de Legendre.

Da mesma forma que Legendre determinou quais são os $n \in \mathbb{N}$ tais que $o\left(n, \mathbb{N}^{2}\right)=4$, Satyanarayana determinou quais são os $n \in \mathbb{N}$ tais que $o\left(n, P_{3}\right)=3$.

Teorema 1.1.6 (Satyanarayana, 1961). Um número $n \in \mathbb{N}$ pode ser escrito como a soma de dois números triangulares se, e somente se, $n$ não é da forma

$$
\frac{p^{2 k+1} s-1}{4}
$$

onde $p$ é um número primo congruente a 3 módulo $4, k \in \mathbb{N}$ e s é congruente a 3 módulo 4 e é primo com p.

Recomendamos [73] para uma prova deste teorema. A primeira demonstração do teorema dos números poligonais de Fermat foi dada por Cauchy.

Teorema 1.1.7 (Cauchy, 1813). Todo número natural pode ser escrito como a soma de $m$ números $m$-gonais, $m \geq 3$. 
Recomendamos [48], p. 27 - 32, para uma prova deste teorema. A segunda generalização do teorema dos quatro quadrados de Lagrange foi iniciada em 1770. Neste ano, Edward Waring, em seu livro Meditationes Algebraicae, conjecturou que todo número natural pode ser escrito como a soma de quatro quadrados, nove cubos, dezenove quartas potências e que, mais geralmente, $\mathbb{N}^{k}$ é base aditiva, $k \in \mathbb{Z}_{+}$. Este problema ficou conhecido como conjectura de Waring, e a primeira solução foi dada por Hilbert.

Teorema 1.1.8 (Hilbert-Waring, 1909). $\mathbb{N}^{k}$ é base aditiva, $k \in \mathbb{Z}_{+}$.

Recomendamos [63], p. 151 - 162, para uma prova deste teorema. Outro avanço nesta área foi dado por Schnirelmann. Utilizando o conceito de densidade de Schnirelmann (falaremos mais dela na terceira seção deste capítulo), Schnirelmann foi capaz de demonstrar o seguinte resultado.

Teorema 1.1.9 (Schnirelmann-Goldbach, 1930). $\mathbb{P}^{*}$ é uma base aditiva.

Recomendamos [48], p. 177 - 199, para uma prova deste teorema. A partir do teorema de Schnirelmann-Goldbach, vários resultados acerca do conjunto dos números primos foram surgindo. Dentre eles, destacamos dois.

Teorema 1.1.10 (Vinogradov, 1937). Todo impar suficientemente grande pode ser escrito como a soma de três primos.

Teorema 1.1.11 (Chen, 1973). Todo par suficientemente grande pode ser escrito na forma $p_{1}+p_{2} p_{3}$, onde $p_{1}, p_{2}, p_{3} \in \mathbb{P}$.

Para uma prova destes teoremas, recomendamos [48], p. 211 - 230, e [48], p. 271 - 298, respectivamente.

\subsection{Ordem assintótica}

Se $A$ for uma base aditiva, pode acontecer que $\{n \in \mathbb{N} ; o(n, A)=o(A)\}$ seja um conjunto finito. Neste caso, faz sentido perguntar qual é o menor número inteiro $o_{\infty}(A)$ tal que todo natural suficientemente grande pode ser escrito como a soma de $o_{\infty}(A)$ elementos de $A$. Isto justifica a seguinte definição.

Definição 1.2.1. Seja A uma base aditiva. Definimos a ordem assintótica de A por

$$
o_{\infty}(A)=\limsup _{n \in \mathbb{N}} o(n, A)
$$

Assim, é claro que se $A$ for uma base aditiva, então $o_{\infty}(A) \leq o(A)$, mas pode ocorrer a desigualdade estrita. Embora isto não ocorra com o conjunto dos números triangulares nem com o conjunto dos números quadrados (teoremas 1.1.4 e 1.1.6, respectivamente), este fenômeno ocorre com o conjunto dos números $m$-gonais, se $m \geq 5$, conforme demonstrou Legendre. 
Teorema 1.2.2 (Legendre, 1830). Sejam $m \geq 5$ e $n \geq 28(m-2)^{3}$. Se $m$ for impar, então $n$ pode ser escrito como a soma de quatro números m-gonais. Se $m$ for par, então $n$ pode ser escrito como a soma de cinco números m-gonais, tal que pelo menos um destes números é 0 ou 1.

Recomendamos [48], p. 27 - 33, para uma prova deste teorema. Com relação ao conjunto das $k$-ésimas potências, embora este fenômeno não ocorra com $\mathbb{N}$ nem com $\mathbb{N}^{2}$, ele ocorre com $\mathbb{N}^{3}$ e $\mathbb{N}^{4}$.

Teorema 1.2.3 (Wieferich-Kempner, 1909-1912). Todo número natural pode ser escrito como a soma de nove cubos positivos.

Recomendamos [48], p. 37 - 43, para uma prova deste teorema. A versão assintótica do teorema de Wieferich-Kempner é conhecida como teorema dos sete cubos de Linnik.

Teorema 1.2.4 (Linnik, 1943). Todo inteiro suficientemente grande pode ser escrito como a soma de sete cubos positivos.

Recomendamos [48], p. 44 - 49, para uma prova deste teorema. Embora não se saiba se o número sete no teorema dos sete cubos de Linnik possa ser substituído por algum número menor, $o_{\infty}\left(\mathbb{N}^{3}\right)$ não pode ser menor do que quatro, devido à proposição abaixo.

Proposição 1.2.5. Se $n \equiv \pm 4(\bmod 9)$, então $n$ não pode ser escrito como a soma de três cubos inteiros.

Demonstração: Note inicialmente que o cubo de todo número inteiro é congruente a 0,1 ou -1 módulo 9. Assim, qualquer número que possa ser escrito como a soma de três cubos inteiros deve ser congruente a $0, \pm 1, \pm 2$ ou \pm 3 módulo 9.

Teorema 1.2.6 (Balasubramanian-Dress-Deshouillers, 1986 - 1992). Todo número natural pode ser escrito como a soma de 19 quartas potências.

Este resultado foi anunciado em [13] e [14], e uma prova completa foi publicada em [21], [22] e [23]. Recomendamos também a leitura de [24] sobre este resultado. Já a versão assintótica do teorema 1.2.6 foi provada por Davenport.

Teorema 1.2.7 (Davenport, 1939). Todo inteiro suficientemente grande pode ser escrito como a soma de 14 quartas potências, a menos que ele seja congruente a 15 ou 16 módulo 16. Em particular, todo inteiro suficientemente grande pode ser escrito como a soma de 16 quartas potências. Como qualquer número da forma $31 \cdot 16^{n}, n \in \mathbb{N}$, não pode ser escrito como a soma de 15 quartas potências, então $o_{\infty}\left(\mathbb{N}^{4}\right)=16$.

Recomendamos [20] para uma prova deste teorema. Para finalizar esta seção, apresentamos uma generalização natural do teorema de Hilbert-Waring, devida a Kamke. 
Teorema 1.2.8 (Kamke, 1921). Seja $f(x) \in \mathbb{C}[x]$ um polinômio não nulo tal que existe $n_{0} \in \mathbb{N}$ tal que se $n \geq n_{0}$, então $f(n) \in \mathbb{N}$. Então

$$
\{0,1\} \cup\left\{f(n) ; n \geq n_{0}\right\}
$$

é uma base aditiva.

Recomendamos [50], p. 355 - 374, para uma prova deste teorema. Falemos agora um pouco mais sobre a densidade de Schnirelmann.

\subsection{Densidade de Schnirelmann}

Definição 1.3.1. Seja $\{0\} \subseteq A \subseteq \mathbb{N}$. A densidade de Schnirelmann de A é definida por

$$
\sigma(A)=\inf _{n \in \mathbb{Z}_{+}} \frac{A(n)}{n}
$$

A densidade de Schnirelmann tem um papel predominante na teoria aditiva dos números por causa do seguinte resultado.

Teorema 1.3.2 (Schnirelmann, 1930). Seja $\{0\} \subseteq A \subseteq \mathbb{N}$. Então A é uma base aditiva se, e somente se, existe $h \in \mathbb{Z}_{+}$tal que $\sigma(h A)>0$.

Para uma elegante demonstração deste teorema, veja [48], p. 191 - 195. Note que, embora $\sigma\left(\mathbb{P}^{*}\right)=0$, Schnirelmann conseguiu mostrar que existe $h \in \mathbb{Z}_{+}$tal que $\sigma\left(h \mathbb{P}^{*}\right)>0$, demonstrando assim o teorema 1.1.9. A densidade de Schnirelmann tem boas propriedades. A primeira delas foi descoberta pelo próprio Schnirelmann.

Teorema 1.3.3 (Schnirelmann, 1933). Sejam $A, B \subseteq \mathbb{N}$ tais que $0 \in A \cap B$. Se $\sigma(A)=\alpha$ e $\sigma(B)=\beta$, então

$$
\sigma(A+B) \geq \alpha+\beta-\alpha \beta
$$

Recomendamos [48], p. 191 - 194, para uma prova desta desigualdade. Entretanto, em todos os casos estudados, o fator $\alpha \beta$ da desigualdade de Schnirelmann podia ser omitido. Isto ficou conhecido como a conjectura $\alpha+\beta$, que foi provada por Henry B. Mann.

Teorema 1.3.4 (Mann, 1942). Sejam $A, B \subseteq \mathbb{N}$ tais que $0 \in A \cap B$. Se $\sigma(A)=\alpha$ e $\sigma(B)=\beta$, então

$$
\sigma(A+B) \geq \min \{1, \alpha+\beta\}
$$

Recomendamos [10] ou [54] para uma prova da desigualdade de Mann. Em particular, a desigualdade de Mann permite obter o seguinte limitante para a ordem de um conjunto $\{0\} \subseteq A \subseteq \mathbb{N}$ tal que $\sigma(A)>0$. 
Corolário 1.3.5. Seja $\{0\} \subseteq A \subseteq \mathbb{N}$. Se $\sigma(A)>0$, então

$$
o(A) \leq\left\lceil\frac{1}{\sigma(A)}\right\rceil .
$$

Note que a desigualdade de Mann não pode ser melhorada. Basta tomar

$$
A=B=\{0,1,11,12,13,14,15, \ldots\} .
$$

Para finalizar esta seção, enunciamos os teoremas de Romanoff.

Teorema 1.3.6 (Romanoff, 1934). Seja $a \in \mathbb{N}, a \geq 2$.

- Se $R_{a}=\{0,1\} \cup\left\{p+a^{n} ; p \in \mathbb{P}, n \in \mathbb{Z}_{+}\right\}$, então $\sigma\left(R_{a}\right)>0$.

- Se $S_{a}=\{0,1\} \cup\left\{p+n^{a} ; p \in \mathbb{P}, n \in \mathbb{Z}_{+}\right\}$, então $\sigma\left(S_{a}\right)>0$.

Recomendamos [48], p. 199 - 204, para uma prova do primeiro item do teorema 1.3.6, ou o artigo [68] do próprio Romanoff para uma prova das duas afirmações do teorema.

\subsection{Componentes essenciais e a desigualdade de Plünnecke}

Em 1933, Khinchin provou o seguinte teorema:

Teorema 1.4.1 (Khinchin, 1933). Seja $A \subseteq \mathbb{N}$ tal que $0<\sigma(A)<1$. Então

$$
\sigma\left(A+\mathbb{N}^{2}\right)>\sigma(A) .
$$

Recomendamos [42] para uma demonstração deste teorema. O teorema de Khinchin deu origem à seguinte definição.

Definição 1.4.2. Dizemos que $B \subseteq \mathbb{N}$ é uma componente essencial se

$$
\sigma(A+B)>\sigma(A)
$$

$\forall A \subseteq \mathbb{N}$ tal que $0<\sigma(A)<1$

Assim, o teorema de Khinchin dá o primeiro exemplo não trivial de uma componente essencial. Como corolário da desigualdade de Schnirelmann, o seguinte resultado vale.

Corolário 1.4.3. Se $\{0\} \subseteq B \subseteq \mathbb{N}$ e $\sigma(B)>0$, então $B$ é uma componente essencial. 
Khinchin:

Em 1935, Erdös provou a seguinte desigualdade, que generaliza o teorema de

Teorema 1.4.4 (Erdös, 1935). Se $B$ for uma base aditiva de ordem $h$ e $A \subseteq \mathbb{N}$ é tal que $0<\sigma(A)<1$, então

$$
\sigma(A+B) \geq \sigma(A)+\frac{\sigma(A)(1-\sigma(A))}{2 h}>\sigma(A) .
$$

Recomendamos [26] para uma prova deste teorema.

Corolário 1.4.5. Toda base aditiva é uma componente essencial.

Finalmente, Plünnecke melhorou a desigualdade de Erdös.

Teorema 1.4.6 (Plünnecke, 1970). Se $B$ for uma base aditiva de ordem $h$ e $A \subseteq \mathbb{N}$ é tal que $0<\sigma(A)<1$, então

$$
\sigma(A+B) \geq \sigma(A)^{1-1 / h}
$$

Recomendamos [49], p. 201 - 226, para uma prova da desigualdade de Plünnecke.

Proposição 1.4.7. A desigualdade de Plünnecke implica a desigualdade de Erdös.

Demonstração: (Seguindo [49], p. 226.) É suficiente mostrar que

$$
x^{1-1 / h} \geq x+\frac{x(1-x)}{2 h}, \forall x \in(0,1), h \in \mathbb{Z}_{+},
$$

ou, equivalentemente,

$$
f(x)=x^{1 / h}\left(1+\frac{1-x}{2 h}\right) \leq 1, \forall x \in(0,1), h \in \mathbb{Z}_{+} .
$$

Como $f(0)=0$ e $f(1)=1$, é suficiente mostrar que $f$ é crescente em $(0,1)$. Com efeito,

$$
f^{\prime}(x)=\frac{x^{1 / h-1}[h(2-x)+(1-x)]}{2 h^{2}}>0, \forall x \in(0,1), h \in \mathbb{Z}_{+},
$$

o que termina a prova desta proposição.

É importante citar que o expoente $1-1 / h$ na desigualdade de Plünnecke é o melhor possível. Um passo-a-passo de como isso pode ser demonstrado se encontra no exercício 10 do capítulo 7 de [49], p. 228 - 229.

Vale a recíproca do corolário 1.4.5? Linnik mostrou que não.

Teorema 1.4.8 (Linnik, 1942). Existe uma componente essencial que não é uma base aditiva. 
Recomendamos [47] para uma prova deste teorema. Assim, é natural perguntarmos: se $A$ for uma componente essencial, existe algum limitante inferior para a função $A(n)$ ? Ruzsa respondeu esta pergunta em 1987.

Teorema 1.4.9 (Ruzsa, 1987). Se A for uma componente essencial, então existe $\varepsilon>0$ tal que

$$
A(n) \gg(\ln (n))^{1+\varepsilon}
$$

Além disso, dado $\varepsilon>0$, existe uma componente essencial $B_{\varepsilon}$ tal que

$$
B_{\varepsilon}(n) \ll(\ln (n))^{1+\varepsilon} .
$$

Recomendamos [71] para uma prova deste teorema.

\subsection{Bases finas}

Da mesma forma que nos perguntamos qual deve ser a magnitude mínima de crescimento do número de elementos de uma componente essencial, podemos nos perguntar qual deve ser a magnitude mínima de crescimento do número de elementos de uma base aditiva. Um simples argumento combinatorial nos dá a resposta.

Proposição 1.5.1. Se A for uma base aditiva de ordem $h$, então existe $c>0$ tal que

$$
A(n) \geq c \sqrt[h]{n}, \forall n \in \mathbb{Z}_{+}
$$

Demonstração: (Seguindo [51].) Seja $n \in \mathbb{Z}_{+}$. Como $A$ é uma base aditiva de ordem $h$, para cada inteiro $m \in A \cap[1, n]$ existem $a_{1, m}, \ldots, a_{h, m} \in A \cap[0, n]$ tais que

$$
m=a_{1, m}+\ldots+a_{h, m} .
$$

Como $A$ contém $A(n)+1$ inteiros menores do que ou iguais a $n$, e como o número de maneiras de escolher $h$ elementos com repetições de um conjunto com $A(n)+1$ elementos é

$$
\left(\begin{array}{c}
A(n)+h \\
h
\end{array}\right)
$$

então

$$
\left(\begin{array}{c}
A(n)+h \\
h
\end{array}\right) \geq n
$$

Mas

$$
\left(\begin{array}{c}
A(n)+h \\
h
\end{array}\right) \leq \frac{(A(n)+h)^{h}}{h !},
$$

$\log 0$

$$
n \leq \frac{(A(n)+h)^{h}}{h !}
$$

o que implica

$$
A(n) \geq \sqrt[h]{n h !}-h \gg \sqrt[h]{n},
$$

o que, por sua vez, é equivalente à tese. 
Definição 1.5.2. Dizemos que uma base aditiva $A$ de ordem $h$ é fina se existe $c^{\prime}>0$ tal que $A(n) \leq c^{\prime} \sqrt[h]{n}, \forall n \in \mathbb{Z}_{+}$.

Portanto, se $A$ for uma base aditiva fina de ordem $h$, existem constantes $c, c^{\prime}>0$ tais que

$$
c \sqrt[h]{n} \leq A(n) \leq c^{\prime} \sqrt[h]{n}, \forall n \in \mathbb{Z}_{+}
$$

Dessa forma, é natural nos perguntar: existem bases aditivas finas? Em 1937, Raikov e Störh foram os primeiros a dar, de forma independente, exemplos de bases aditivas finas.

Teorema 1.5.3 (Raikov-Stöhr, 1937). Para cada $h \in \mathbb{N}$ existe uma base aditiva fina $F_{h}$ de ordem $h$.

Recomendamos [51] para uma prova deste teorema. Denominaremos $F_{h}$ de base aditiva fina de Raikov-Stöhr de ordem $h$. As bases aditivas finas desempenharão um papel importante nesta tese.

\subsection{Teoremas de P.A.'s}

Outra parte da teoria aditiva dos números envolve entender as relações entre subconjuntos quaisquer de $\mathbb{N}$ e progressões aritméticas. O primeiro resultado que gostaríamos de destacar aqui é o de Erdös.

Teorema 1.6.1 (Erdös, 1950). Existe uma progressão aritmética infinita de inteiros positivos impares, nenhum dos quais pode ser escrito na forma $p+2^{n}$, onde $p \in \mathbb{P}$ e $n \in \mathbb{Z}_{+}$.

Recomendamos [48], p. 204 - 207, para uma prova deste teorema. Vale a pena comparar o teorema 1.6.1 com o teorema 1.3.6. Em 1927, van der Waerden provou seu teorema.

Teorema 1.6.2 (van der Waerden, 1927). Sejam $k, r \in \mathbb{Z}_{+}$. Então existe um inteiro positivo $W$ tal que, independente de como o conjunto $\{1,2, \ldots, W\}$ seja particionado em $r$ subconjuntos, pelo menos um deles vai conter uma progressão aritmética de tamanho $k$.

Recomendamos [43], p. 11 - 17, para uma prova deste teorema. Dados $k, r \in \mathbb{Z}_{+}$, o menor inteiro $W$ que satisfaz o teorema de van der Waerden é chamado número de van der Waerden de $(k, r)$, e é denotado por $W(k, r)$. Note que o teorema de van der Waerden é equivalente ao enunciado de que se

$$
\mathbb{Z}_{+}=\bigcup_{i=1}^{r} C_{i}
$$

então algum $C_{i}$ contém progressões aritméticas arbitrariamente grandes. O teorema de van der Waerden foi generalizado da seguinte forma por Szemerédi. 
Teorema 1.6.3 (Szemerédi, 1975). Sejam $k \in \mathbb{Z}_{+}$e $\delta>0$. Então existe um inteiro positivo $N=N(k, \delta)$ tal que qualquer subconjunto do conjunto $\{1,2, \ldots, N\}$ de tamanho pelo menos $\delta N$ contém uma progressão aritmética de tamanho $k$.

Note que o teorema de Szemerédi generaliza o teorema de van der Waerden: basta tomar

$$
W(k, r)=N(k, 1 / r) .
$$

Perceba que o teorema de Szemerédi é equivalente ao fato de que se $A \subseteq \mathbb{Z}_{+}$é tal que

$$
\limsup _{n \in \mathbb{Z}_{+}} \frac{A(n)}{n}>0
$$

então $A$ contém progressões aritméticas arbitrariamente grandes. Existem hoje várias provas deste teorema. Em particular, citamos a prova de Harry Furstenberg usando teoria ergódica (veja [29]), a prova de Timothy Gowers usando análise de Fourier e combinatória (veja [31]) e a própria prova combinatória de Szemerédi (veja [81]).

Recentemente, Ben Green e Terence Tao estenderam o teorema de Szemerédi.

Teorema 1.6.4 (Green-Tao, 2004). O conjunto dos números primos contém progressões aritméticas arbitrariamente grandes.

Recomendamos [33] para uma prova deste teorema. Note que o teorema de Green-Tao é, de fato, uma extensão do teorema de Szemerédi, uma vez que

$$
\lim _{n \rightarrow \infty} \frac{\mathbb{P}(n)}{n}=0 .
$$

Vale citar aqui que as quatro maiores progressões aritméticas conhecidas consistindo apenas de primos são dadas por

- $3486107472997423+371891575525470 n$, para $n \in\{0,1, \ldots, 25\}$;

- $43142746595714191+5283234035979900 n$, para $n \in\{0,1, \ldots, 25\}$;

- $136926916457315893+9843204333812850 n$, para $n \in\{0,1, \ldots, 25\}$;

- $161004359399459161+10644900609172830 n$, para $n \in\{0,1, \ldots, 25\}$.

Recomendamos o site [6] para outros recordes nesta área. Vejamos agora alguns problemas em aberto da teoria aditiva dos números.

\subsection{Problemas em aberto}

O problema em aberto mais antigo da teoria aditiva dos números (que eu conheça), e que muito me motivou a estudar teoria dos números é a célebre conjectura de Goldbach. 
Conjectura 1.7.1 (Goldbach, 1742). Todo número par maior que dois pode ser expresso como a soma de dois primos.

A conjectura de Goldbach foi proposta numa carta (veja [30]) escrita em 7 de junho de 1742 pelo matemático Christian Goldbach e endereçada a Leonhard Euler. Apesar de todos os esforços desprendidos até hoje, não se sabe o valor lógico dela. Resultados computacionais mostram que a conjectura de Goldbach é verdadeira para todo par menor do que ou igual a $4 \cdot 10^{18}$ (veja [79]). Recentemente (em $2012 \mathrm{e}$ 2013), H. A. Helfgott disponibilizou três artigos no sistema arXiv ([34], [35] e [36]) contendo uma prova de que todo inteiro ímpar maior do que 7 pode ser escrito como a soma de três primos. Recomendamos [37] para uma versão unificada e completa deste resultado.

Seguindo em ordem cronológica, outro problema antigo ainda em aberto é a solução exata do problema de Waring.

Conjectura 1.7.2. $o\left(\mathbb{N}^{k}\right)=2^{k}+\left\lfloor(3 / 2)^{k}\right\rfloor-2, k \in \mathbb{Z}_{+}$.

O número que aparece no lado direito da igualdade anterior não é mágico.

Proposição 1.7.3. Seja $k \in \mathbb{Z}_{+}$e defina $n_{k}=2^{k}\left\lfloor(3 / 2)^{k}\right\rfloor-1$. Então

$$
o\left(n_{k}, \mathbb{N}^{k}\right)=2^{k}+\left\lfloor(3 / 2)^{k}\right\rfloor-2 .
$$

Demonstração: Note inicialmente que $n_{k}<3^{k}$. Dessa forma, para escrevermos $n_{k}$ como soma de elementos de $\mathbb{N}^{k}$, só podemos usar os números 0,1 e $2^{k}$. Como

$$
n_{k}=2^{k}\left\lfloor(3 / 2)^{k}\right\rfloor-1=2^{k}\left(\left\lfloor(3 / 2)^{k}\right\rfloor-1\right)+2^{k}-1=\underbrace{2^{k}+\cdots+2^{k}}_{\left\lfloor(3 / 2)^{k}\right\rfloor-1 \text { vezes }}+\underbrace{1+\cdots+1}_{2^{k}-1 \text { vezes }}
$$

então

$$
o\left(n_{k}, \mathbb{N}^{k}\right)=\left\lfloor(3 / 2)^{k}\right\rfloor-1+2^{k}-1=2^{k}+\left\lfloor(3 / 2)^{k}\right\rfloor-2 .
$$

Ademais, combinando os trabalhos de Dickson, Pillai, Rubugunday, Niven, Chen, Balasubramanian, Deshouillers e Dress (veja [63], p. 161 - 162), temos o seguinte resultado.

Teorema 1.7.4. Denote por $\langle x\rangle$ a parte fracionária de $x$, isto é, $\langle x\rangle=x-\lfloor x\rfloor$. Se

$$
2^{k}\left\langle(3 / 2)^{k}\right\rangle+\left\lfloor(3 / 2)^{k}\right\rfloor \leq 2^{k},
$$

então a conjectura 1.7.2 é verdadeira para $k$.

Mais ainda pode ser dito sobre a conjectura 1.7.2.

Teorema 1.7.5 (Mahler, 1957). A desigualdade

$$
2^{k}\left\langle(3 / 2)^{k}\right\rangle+\left\lfloor(3 / 2)^{k}\right\rfloor \leq 2^{k}, \quad k \in \mathbb{Z}_{+},
$$

é falsa no máximo um número finito de vezes. 
Recomendamos [52] para uma prova deste teorema. Em particular, a conjectura 1.7.2 é falsa no máximo um número finito de vezes. Além disso, Kubina e Wunderlich mostraram em [44] que todo $k \leq 471600000$ satisfaz a desigualdade do teorema 1.7.4.

Tão importante quanto a conjectura 1.7.2 é a determinação exata de $o_{\infty}\left(\mathbb{N}^{k}\right)$, $k \in \mathbb{Z}_{+}$, e, em especial, $o_{\infty}\left(\mathbb{N}^{3}\right)$.

Problema 1.7.6. Determine $o_{\infty}\left(\mathbb{N}^{k}\right), k \in \mathbb{Z}_{+}$. Em particular, é verdade que $o_{\infty}\left(\mathbb{N}^{3}\right)=4$ ?

Dois outros problemas muito antigos em teoria aditiva dos números são as conjecturas de Pollock (veja [64]).

Conjectura 1.7.7 (Pollock, 1850). Sejam

$$
\begin{gathered}
\mathcal{T}=\left\{\frac{n(n+1)(n+2)}{6} ; n \in \mathbb{N}\right\}=\{0,1,4,10,20,35,56,84,120,165,220, \ldots\} \\
\mathcal{O}=\left\{\frac{n\left(2 n^{2}+1\right)}{3} ; n \in \mathbb{N}\right\}=\{0,1,6,19,44,85,146,231,344,489,670,891, \ldots\}
\end{gathered}
$$

os conjuntos dos números tetraédricos e octaédricos, respectivamente. Então $\mathcal{T}$ é uma base aditiva de ordem 5 e $\mathcal{O}$ é uma base aditiva de ordem 7.

É claro que, devido ao teorema 1.2.8, tanto $\mathcal{T}$ quanto $\mathcal{O}$ são bases aditivas. O problema é a determinação exata da ordem de cada uma destas bases. Até agora, o melhor resultado é o de Watson.

Teorema 1.7.8 (Watson, 1952). $\mathcal{T}$ é uma base aditiva e $o(\mathcal{T}) \leq 8$.

Recomendamos [84] para uma prova deste teorema. O próximo problema que enunciaremos aqui versa sobre a determinação exata dos números de van der Waerden.

Problema 1.7.9. Dados $k, r \in \mathbb{Z}_{+}$, determine $W(k, r)$.

Existem alguns limitantes superiores conhecidos (veja [31]). Sobre o número de representações de um número $n \in \mathbb{N}$ como soma de $h$ elementos de uma base aditiva de ordem $h$, temos a conjectura de Erdös-Turán.

Conjectura 1.7.10 (Erdös-Turán, 1941). Seja A base aditiva de ordem assintótica $h \geq 2$ e denote por $r(n, A)$ o número de representações de um inteiro $n$ como soma de $h$ elementos de $A$. Então $\limsup _{n \rightarrow \infty} r(n, A)=\infty$.

No artigo original de Erdös e Turán (veja [28]), esta conjectura é enunciada apenas para bases aditivas de ordem assintótica igual a 2, mas sua generalização para bases aditivas de ordem assintótica qualquer é natural e é esta versão que escolhemos enunciar do problema.

Outro problema, também de Erdös, bastante famoso e ainda sem sinal de solução, é a conjectura de Erdös sobre progressões aritméticas (veja [27]). 
Conjectura 1.7.11 (Erdös, 1976). Seja $A \subseteq \mathbb{Z}_{+}$tal que

$$
\sum_{a \in A} \frac{1}{a}=\infty
$$

Então A contém progressões aritméticas arbitrariamente grandes.

Note que a conjectura de Erdös implica o teorema 1.6.4, pois

$$
\sum_{p \in \mathbb{P}} \frac{1}{p}=\infty
$$

assim como o teorema de Szemerédi, devido à proposição abaixo.

Proposição 1.7.12. Seja $A \subseteq \mathbb{Z}_{+}$tal que

$$
\limsup _{n \in \mathbb{Z}_{+}} \frac{A(n)}{n}>0 \text {. }
$$

Então

$$
\sum_{a \in A} \frac{1}{a}=\infty
$$

Demonstração: Seja

$$
l=\limsup _{n \in \mathbb{Z}_{+}} \frac{A(n)}{n} .
$$

Como $l>0$, então existe uma sequência estritamente crescente de inteiros positivos $\left\{n_{j}\right\}_{j \in \mathbb{N}}$ tal que $n_{0}=1$ e $n_{j}$ satisfaz

$$
\frac{A\left(n_{j}\right)}{n_{j}} \geq \frac{l}{2} \quad \text { e } \quad n_{j} \geq \frac{4 n_{j-1}}{l}, \quad \forall j \in \mathbb{Z}_{+} .
$$

Então

$$
\begin{aligned}
\sum_{a \in A} \frac{1}{a} & \geq \sum_{j=1}^{\infty} \sum_{a \in A \cap\left(n_{j-1}, n_{j}\right]} \frac{1}{a} \geq \sum_{j=1}^{\infty} \frac{\#\left(A \cap\left(n_{j-1}, n_{j}\right]\right)}{n_{j}} \geq \sum_{j=1}^{\infty} \frac{A\left(n_{j}\right)-n_{j-1}}{n_{j}} \geq \\
& \geq \sum_{j=1}^{\infty}\left(\frac{l}{2}-\frac{l}{4}\right)=\sum_{j=1}^{\infty} \frac{l}{4}=\infty .
\end{aligned}
$$

Para finalizar este capítulo, enunciamos o chamado problema das constantes das bases aditivas finas, de caráter mais recente. Vimos na seção 5 deste capítulo que se $A$ for uma base aditiva fina de ordem $h$, então existem constantes $c, c^{\prime}>0$ tais que

$$
c \sqrt[h]{n} \leq A(n) \leq c^{\prime} \sqrt[h]{n}, \forall n \in \mathbb{Z}_{+}
$$

Assim, poderíamos perguntar: fixado $h \in \mathbb{N}$, pelo menos quantos elementos uma base aditiva de ordem $h$ deve conter? Mais especificamente (veja [51]), sejam

$$
\alpha_{h}=\inf _{\substack{A \in \mathcal{A} \\ o(A)=h}} \liminf _{n \rightarrow \infty} \frac{A(n)}{\sqrt[h]{n}} \quad \text { e } \quad \beta_{h}=\inf _{\substack{A \in \mathcal{A} \\ o(A)=h}} \limsup _{n \rightarrow \infty} \frac{A(n)}{\sqrt[h]{n}} .
$$


Problema 1.7.13 (Nathanson, 2009). Determine exatamente $\alpha_{h}$ e $\beta_{h}, \forall h \geq 2$.

Nesta direção, temos o resultado de Stöhr.

Teorema 1.7.14 (Stöhr, 1955). $\beta_{h} \geq \frac{\sqrt[h]{h !}}{\Gamma(1+1 / h)}$, onde $\Gamma(x)$ é a função Gamma.

Recomendamos [77] para uma prova deste teorema. Em particular, se $A$ for uma base aditiva de ordem 2 , então

$$
\limsup _{n \rightarrow \infty} \frac{A(n)}{\sqrt{n}} \geq \sqrt{\frac{8}{\pi}}
$$

Vale mencionar que mesmo os números $\alpha_{2}$ e $\beta_{2}$ são desconhecidos. 


\section{Capítulo 2}

\section{O espaço métrico das bases aditivas}

Este capítulo visa apresentar a métrica $d$ em $\mathcal{A}$ sobre a qual desenvolveremos nosso trabalho. Objetiva demonstrar as propriedades topológicas básicas do espaço métrico $(\mathcal{A}, d)$, assim como caracterizá-lo topologicamente. Também apresenta alguns conceitos novos que serão fortemente usados nos capítulos 3 e 4 desta tese. Para as referências básicas sobre espaços métricos recomendamos [46] e [60].

\section{$2.1 \quad$ Métrica em $\mathcal{A}$}

Teorema 2.1.1. A função

$$
\begin{array}{rlc}
d: \mathcal{A} \times \mathcal{A} & \rightarrow & {[0, \infty)} \\
(A, B) & \mapsto & |o(A)-o(B)|+\sum_{\alpha \in A \triangle B} \frac{1}{\alpha^{2}}
\end{array}
$$

é uma métrica em $\mathcal{A}$.

Demonstração: Nós só precisamos provar a desigualdade triangular. Para isto, sejam $A, B, C \in \mathcal{A}$. Como

$$
A \triangle C \subseteq(A \triangle B) \cup(B \triangle C),
$$

então

$$
\begin{aligned}
d(A, C) & =|o(A)-o(C)|+\sum_{\alpha \in A \triangle C} \frac{1}{\alpha^{2}} \leq \\
& \leq|o(A)-o(B)|+\sum_{\alpha \in A \triangle B} \frac{1}{\alpha^{2}}+|o(B)-o(C)|+\sum_{\alpha \in B \triangle C} \frac{1}{\alpha^{2}}= \\
& =d(A, B)+d(B, C) .
\end{aligned}
$$


Note que a função

$$
\begin{array}{rlc}
\widehat{d}: \mathcal{A} \times \mathcal{A} & \rightarrow & {[0, \infty)} \\
(A, B) & \mapsto & |o(A)-o(B)|
\end{array}
$$

é uma pseudométrica. Ademais, o número 2 usado em

$$
\sum_{\alpha \in A \triangle B} \frac{1}{\alpha^{2}}
$$

pode ser substituído por qualquer outro número $w \in(0, \infty)$ tal que

$$
\sum_{n=2}^{\infty} \frac{1}{n^{w}}<1
$$

O motivo disto ficará claro no decorrer desta tese. Para começar a termos familiaridade com a métrica $d$, vamos dar dois exemplos do cálculo da distância entre duas bases aditivas. O primeiro exemplo envolve duas bases muito conhecidas: $\mathbb{N}$ e $\mathbb{N}^{2}$.

$$
\begin{aligned}
d\left(\mathbb{N}, \mathbb{N}^{2}\right) & =\left|o(\mathbb{N})-o\left(\mathbb{N}^{2}\right)\right|+\sum_{\alpha \in \mathbb{N} \triangle \mathbb{N}^{2}} \frac{1}{\alpha^{2}}=|1-4|+\sum_{\alpha \in \mathbb{N}-\mathbb{N}^{2}} \frac{1}{\alpha^{2}}= \\
& =3+\sum_{\alpha=2}^{\infty} \frac{1}{\alpha^{2}}-\sum_{\alpha=2}^{\infty} \frac{1}{\alpha^{4}}=3+\left(\frac{\pi^{2}}{6}-1\right)-\left(\frac{\pi^{4}}{90}-1\right)=3+\frac{\pi^{2}}{6}-\frac{\pi^{4}}{90}
\end{aligned}
$$

O outro exemplo que daremos aqui envolve um teorema bastante profundo em teoria dos números. Vamos calcular a distância do conjunto dos cubos, $\mathbb{N}^{3}$, até o conjunto dos números triangulares, $P_{3}$.

$$
d\left(\mathbb{N}^{3}, P_{3}\right)=\left|o\left(\mathbb{N}^{3}\right)-o\left(P_{3}\right)\right|+\sum_{\alpha \in \mathbb{N}^{3} \triangle P_{3}} \frac{1}{\alpha^{2}}=|9-3|+\sum_{\alpha \in \mathbb{N}^{3} \triangle P_{3}} \frac{1}{\alpha^{2}} .
$$

Para prosseguir, precisamos resolver a equação diofantina

$$
x^{3}=\frac{y(y+1)}{2} .
$$

Rearranjando esta equação, obtemos

$$
(2 y+1)^{2}-(2 x)^{3}=1 .
$$

Pelo teorema de Mihailescu ${ }^{1}, x=y=0$ ou $x=y=1$. Logo

$$
\mathbb{N}^{3} \triangle P_{3}=\left(\mathbb{N}^{3}-\{0,1\}\right) \cup\left(P_{3}-\{0,1\}\right) .
$$

\footnotetext{
${ }^{1}$ Em 1844 (veja [15]), Eugène Charles Catalan perguntou se 8 e 9 são as únicas potências consecutivas de números inteiros positivos. Isto foi provado em 2002 por Preda Mihailescu. Veja [57] para o artigo original de Mihailescu ou o livro [74] para uma apresentação bastante completa deste problema. Deve ser notado, entretanto, que não se precisa de fato do teorema de Mihailescu para calcular esta distância; basta resolver a equação diofantina
}

$$
r^{2}-s^{3}=1 .
$$

Existem métodos mais simples para resolver esta equação diofantina em particular. 
Portanto

$$
\begin{aligned}
d\left(\mathbb{N}^{3}, P_{3}\right) & =6+\sum_{\alpha \in \mathbb{N}^{3}-\{0,1\}} \frac{1}{\alpha^{2}}+\sum_{\alpha \in P_{3}-\{0,1\}} \frac{1}{\alpha^{2}}= \\
& =6+\sum_{\alpha=2}^{\infty} \frac{1}{\alpha^{6}}+\sum_{\alpha=2}^{\infty} \frac{4}{\alpha^{2}(\alpha+1)^{2}}= \\
& =6+\sum_{\alpha=2}^{\infty} \frac{1}{\alpha^{6}}+\sum_{\alpha=2}^{\infty}\left(\frac{4}{\alpha^{2}}+\frac{4}{(\alpha+1)^{2}}+\frac{8}{\alpha+1}-\frac{8}{\alpha}\right)= \\
& =6+\left(\frac{\pi^{6}}{945}-1\right)+4\left(\frac{\pi^{2}}{6}-1\right)+4\left(\frac{\pi^{2}}{6}-1-\frac{1}{4}\right)-4= \\
& =6+\frac{\pi^{6}}{945}+\frac{4 \pi^{2}}{3}-14
\end{aligned}
$$

Se $x \in \mathbb{Z}_{+}$, denotaremos

$$
\overleftarrow{x}=\{y \in \mathbb{N} ; 1 \leq y \leq x\}
$$

para simplificar a notação.

Lema 2.1.2. Se $d(A, B) \in \mathbb{N}$, então $A=B$. Além disso, se $x \in \mathbb{Z}_{+} e$

$$
d(A, B)<\frac{1}{x^{2}}
$$

ent $\tilde{a} o A \cap \overleftarrow{x}=B \cap \overleftarrow{x}$

Demonstração: Se $d(A, B) \in \mathbb{N}$, então

$$
\sum_{\alpha \in A \triangle B} \frac{1}{\alpha^{2}}=0
$$

pois $|o(A)-o(B)| \in \mathbb{N}$ e

$$
0 \leq \sum_{\alpha \in A \triangle B} \frac{1}{\alpha^{2}} \leq \sum_{\alpha=2}^{\infty} \frac{1}{\alpha^{2}}=\frac{\pi^{2}}{6}-1<1 .
$$

Logo $A=B$. Já se $x \in \mathbb{Z}_{+}$é tal que

$$
A \cap \overleftarrow{x} \neq B \cap \overleftarrow{x}
$$

então existe $y \in(A \triangle B) \cap \overleftarrow{x}$, o que implica

$$
d(A, B) \geq \frac{1}{y^{2}} \geq \frac{1}{x^{2}} .
$$

Proposição 2.1.3. A função

$$
\begin{aligned}
o: \mathcal{A} & \rightarrow \mathbb{Z}_{+} \\
A & \mapsto o(A)
\end{aligned}
$$

é uma contração fraca, mas não é uma contração. 
Demonstração: A primeira afirmação segue diretamente da definição da métrica $d$. Para a segunda afirmação, suponha por absurdo que exista $c \in[0,1)$ tal que

$$
|o(A)-o(B)| \leq c \cdot d(A, B), \forall A, B \in \mathcal{A} .
$$

Claramente $c \neq 0$, pois se este fosse o caso todas as bases teriam a mesma ordem. Assim, $0<c<1$. Seja $x \in \mathbb{N}-\{0,1\}$ tal que

$$
c \cdot\left(1+\frac{1}{x^{2}}\right)<1 \text {. }
$$

Se $A=\mathbb{N}$ e $B=\mathbb{N}-\{x\}$, então

$$
1=|o(A)-o(B)| \leq c \cdot d(A, B)=c \cdot\left(1+\frac{1}{x^{2}}\right)<1
$$

absurdo.

O lema abaixo será usado no futuro.

Lema 2.1.4. Seja $\left\{A_{n}\right\}_{n \in \mathbb{N}}$ uma sequência de subconjuntos de $\mathbb{Z}_{+}$. Suponha que exista $A \subseteq \mathbb{Z}_{+}$tal que dado $\varepsilon>0$ existe $n_{0} \in \mathbb{Z}_{+}$tal que se $n \geq n_{0}$, então

$$
\sum_{\alpha \in A_{n} \triangle A} \frac{1}{\alpha^{2}}<\varepsilon .
$$

Então

$$
A=\bigcup_{m=0}^{\infty} \bigcap_{n=m}^{\infty} A_{n}
$$

Demonstração: Seja

$$
B=\bigcup_{m=0}^{\infty} \bigcap_{n=m}^{\infty} A_{n}
$$

Vamos mostrar que $A \subseteq B$ e que $A \supseteq B$.

$(\subseteq)$ Seja $x \in A$. Por hipótese, dado

$$
\varepsilon=\frac{1}{x^{2}}
$$

existe $n_{0} \in \mathbb{N}$ tal que se $n \geq n_{0}$, então

$$
\sum_{\alpha \in A_{n} \triangle A} \frac{1}{\alpha^{2}}<\frac{1}{x^{2}} .
$$

Em particular,

$$
A \cap \overleftarrow{x}=A_{n} \cap \overleftarrow{x}, \forall n \geq n_{0}
$$

Logo $n \in B$.

$(\supseteq)$ Seja $x \in B$. Então existe $m \in \mathbb{N}$ tal que se $n \geq m$, então $x \in A_{n}$. Por hipótese, dado

$$
\varepsilon=\frac{1}{x^{2}}
$$


existe $n_{0} \in \mathbb{N}$ tal que se $n \geq n_{0}$, então

$$
\sum_{\alpha \in A_{n} \triangle A} \frac{1}{\alpha^{2}}<\frac{1}{x^{2}} .
$$

Logo,

$$
A \cap \overleftarrow{x}=A_{n} \cap \overleftarrow{x}, \forall n \geq n_{0}
$$

Se $k=\max \left\{m, n_{0}\right\}$, então $x \in A_{k}$, o que implica que $x \in A$.

Se $h \in \mathbb{Z}_{+}$, denotaremos

$$
\mathcal{A}_{h}=\{A \in \mathcal{A} ; o(A)=h\}
$$

para simplificar a notação.

Proposição 2.1.5. Os conjuntos $\mathcal{A}_{h}$ satisfazem as seguintes propriedades:

1. $\mathcal{A}_{h}$ é aberto e fechado em $\mathcal{A}$.

2. $\mathcal{A}_{h}$ é totalmente limitado.

3. $\partial\left(\mathcal{A}_{h}\right)=\emptyset$, onde $\partial\left(\mathcal{A}_{h}\right)$ representa a fronteira de $\mathcal{A}_{h}$.

4. $\mathcal{A}_{h}$ é completo se, e somente se, $h=1$.

Demonstração: 1. Como a função

$$
\begin{aligned}
o: \mathcal{A} & \rightarrow \mathbb{Z}_{+} \\
A & \mapsto o(A)
\end{aligned}
$$

é contínua em $\mathcal{A}$ e $\mathcal{A}_{h}=o^{-1}(\{h\})$, segue que $\mathcal{A}_{h}$ é aberto e fechado em $\mathcal{A}$.

2. Sejam $\varepsilon>0$ e $m \in \mathbb{N}-\{0,1\}$ tal que

$$
\sum_{\alpha>m} \frac{1}{\alpha^{2}}<\varepsilon
$$

Para cada $\Gamma \subseteq\{2, \ldots, m\}$ escolha, se possível, $A_{\Gamma} \in \mathcal{A}_{h}$ tal que

$$
A_{\Gamma} \cap\{2, \ldots, m\}=\Gamma .
$$

Seja $A \in \mathcal{A}_{h}$. Então

$$
A \cap\{2, \ldots, m\}=A_{\Gamma} \cap\{2, \ldots, m\},
$$

para algum $\Gamma \subseteq\{2, \ldots, m\}$. Logo

$$
d\left(A, A_{\Gamma}\right)=\sum_{\alpha \in A \triangle A_{\Gamma}} \frac{1}{\alpha^{2}} \leq \sum_{\alpha>m} \frac{1}{\alpha^{2}}<\varepsilon .
$$

Isto implica que $\mathcal{A}_{h}$ é totalmente limitado. 
3. Suponha que exista $A \in \partial\left(\mathcal{A}_{h}\right)$. Então dado

$$
r=\frac{1}{2}
$$

existem $B_{1} \in \mathcal{A}_{h}$ e $B_{2} \in \mathcal{A}-\mathcal{A}_{h}$ tais que

$$
B_{1}, B_{2} \in \mathcal{B}(A, r) .
$$

Então

$$
d\left(B_{1}, A\right)<\frac{1}{2}
$$

o que implica $o\left(B_{1}\right)=o(A)$, e

$$
d\left(B_{2}, A\right)<\frac{1}{2}
$$

o que implica $o\left(B_{2}\right)=o(A)$. Logo $o\left(B_{1}\right)=o\left(B_{2}\right)$, absurdo.

4. Claramente $\mathcal{A}_{1}$ é completo, pois $\mathcal{A}_{1}=\{\mathbb{N}\}$. Agora, para cada $h \geq 2$, nós vamos construir uma sequência de Cauchy

$$
\left\{A_{n}\right\}_{n \in \mathbb{Z}_{+}} \subseteq \mathcal{A}_{h}
$$

tal que $\left\{A_{n}\right\}_{n \in \mathbb{Z}_{+}}$não converge em $\mathcal{A}_{h}$. Fixado $h \geq 2$, considere a sequência $\left\{A_{n}\right\}_{n \in \mathbb{Z}_{+}}$dada por

$$
A_{n}=\{0,1, \ldots, n, h n+1, \ldots\} .
$$

Então

$$
o\left(A_{n}\right)=h, \forall n \in \mathbb{Z}_{+} .
$$

Se $\left\{A_{n}\right\}_{n \in \mathbb{Z}_{+}}$convergisse em $\mathcal{A}_{h}$, existiria $A \in \mathcal{A}_{h}$ tal que $A_{n} \rightarrow A$. Pelo lema 2.1.4,

$$
A=\bigcup_{m=1}^{\infty} \bigcap_{n=m}^{\infty} A_{n}=\mathbb{N},
$$

o que implica $A_{n} \rightarrow \mathbb{N}$, absurdo, pois $o\left(A_{n}\right)=h \geq 2, \forall n \in \mathbb{Z}_{+}$.

Da proposição anterior nós tiramos dois corolários.

Corolário 2.1.6. As seguintes afirmações são equivalentes a respeito de um conjunto $X \subseteq \mathcal{A}$ :

1. $X$ é totalmente limitado.

2. $X$ é limitado.

3. Existe $M>0$ tal que $o(A) \leq M, \forall A \in X$.

Demonstração: (1. $\Rightarrow$ 2.) Trivial.

(2. $\Rightarrow$ 3.) Como $X$ é limitado, existe $r>0$ tal que $X \subseteq \mathcal{B}(\mathbb{N}, r)$. Assim, se $A \in X$ e $M=r+1>0$, então

$$
o(A)=|o(A)-1|+1 \leq d(A, \mathbb{N})+1<r+1=M .
$$


(3. $\Rightarrow 1$.) Se existe $M>0$ tal que $o(A) \leq M, \forall A \in X$, então

$$
X \subseteq \bigcup_{h=1}^{\lfloor M\rfloor} \mathcal{A}_{h},
$$

ou seja, $X$ está contido em um conjunto totalmente limitado. Então $X$ é totalmente limitado.

Corolário 2.1.7. $\mathcal{A}$ não é conexo, nem completo, nem compacto.

Demonstração: $\mathcal{A}$ não é conexo, pois podemos escrever

$$
\mathcal{A}=\mathcal{A}_{1} \cup\left(\bigcup_{h=2}^{\infty} \mathcal{A}_{h}\right)
$$

$\mathcal{A}$ também não é completo; se este fosse o caso, então $\mathcal{A}_{h}$, para qualquer $h \geq 2$, sendo um subconjunto fechado de $\mathcal{A}$, seria completo também. Por fim, como $\mathcal{A}$ não é completo, $\mathcal{A}$ não é compacto.

O lema abaixo dá outro exemplo de um subconjunto aberto e fechado em $\mathcal{A}$.

Lema 2.1.8. Se $\Gamma \subseteq \mathbb{N}$ é finito, então

$$
X=\{A \in \mathcal{A} ; \Gamma \subseteq A\}
$$

é aberto e fechado em $\mathcal{A}$.

Demonstração: O resultado é trivial se $\Gamma \subseteq\{0,1\}$. Assim, suponha que $\Gamma \nsubseteq\{0,1\}$ e seja

$$
x=\max \Gamma \geq 2 .
$$

Para mostrar que $X$ é aberto, seja $A \in X$. Afirmo que

$$
\mathcal{B}\left(A, \frac{1}{x^{2}}\right) \subseteq X
$$

De fato, se

$$
B \in \mathcal{B}\left(A, \frac{1}{x^{2}}\right)
$$

então

$$
d(A, B)<\frac{1}{x^{2}}
$$

o que implica

$$
A \cap \overleftarrow{x}=B \cap \overleftarrow{x}
$$

Logo $\Gamma \subseteq B$, o que implica $B \in X$.

Para mostrar que $X$ é fechado, seja $\left\{A_{n}\right\}_{n \in \mathbb{N}} \subseteq X$ tal que $A_{n} \rightarrow A \in \mathcal{A}$. Precisamos mostrar que $A \in X$. Como $A_{n} \rightarrow A$, dado

$$
\varepsilon=\frac{1}{x^{2}}
$$


existe $n_{0} \in \mathbb{N}$ tal que se $n \geq n_{0}$, então $d\left(A, A_{n}\right)<\frac{1}{x^{2}}$. Em particular,

$$
d\left(A, A_{n_{0}}\right)<\frac{1}{x^{2}}
$$

o que implica

$$
A \cap \overleftarrow{x}=A_{n_{0}} \cap \overleftarrow{x}
$$

Como $A_{n_{0}} \in X$, então $\Gamma \subseteq A_{n_{0}}$, o que implica $\Gamma \subseteq A$ e, portanto, $A \in X$.

De posse do lema 2.1.8 podemos ser mais específicos com respeito à questão de quanto $\mathcal{A}$ é desconexo. Para isto, vamos relembrar a seguinte definição:

Definição 2.1.9. Um espaço topológico $(T, \tau)$ é totalmente separado se dados dois pontos distintos $u, v \in T$, então existem dois conjuntos abertos e disjuntos $U, V \in \tau$ tais que $u \in U, v \in V$ e $T=U \cup V$.

Proposição 2.1.10. $\mathcal{A}$ é totalmente separado. Em particular, $\mathcal{A}$ é totalmente desconexo.

Demonstração: Sejam $A_{1}, A_{2} \in \mathcal{A}$ tais que $A_{1} \neq A_{2}$. Então $A_{1} \triangle A_{2} \neq \emptyset$. Seja $x=\min \left(A_{1} \triangle A_{2}\right)$ e suponha sem perda de generalidade que $x \in A_{1}$. Escreva

$$
\mathcal{A}=\{A \in \mathcal{A} ; x \in A\} \cup\{A \in \mathcal{A} ; x \notin A\} .
$$

Então $A_{1} \in\{A \in \mathcal{A} ; x \in A\}, A_{2} \in\{A \in \mathcal{A} ; x \notin A\}$ e tanto $\{A \in \mathcal{A} ; x \in A\}$ quanto $\{A \in \mathcal{A} ; x \notin A\}$ são subconjuntos abertos de $\mathcal{A}$, pelo lema 2.1.8. Então $\mathcal{A}$ é totalmente separado. Como qualquer espaço topológico totalmente separado é totalmente desconexo, o corolário está provado.

Vamos agora definir dois conceitos que serão muito úteis na caracterização topológica do espaço métrico $(\mathcal{A}, d)$. O ponto central desta caracterização envolve a caracterização de quais subconjuntos de $\mathcal{A}$ são compactos. Este será o objetivo da próxima seção.

\subsection{Primeiro pior caso e pseudocontinuidade}

Definição 2.2.1. Seja $A \in \mathcal{A}$. Definimos o primeiro pior caso de $A$ por

$$
\operatorname{ppc}(A)=\min \{n \in \mathbb{N} ; o(n, A)=o(A)\} .
$$

Exemplo 2.2.2. $\operatorname{ppc}\left(\mathbb{N}^{2}\right)=7, \operatorname{ppc}\left(\mathbb{N}^{3}\right)=23$ e $\operatorname{ppc}(A)=0$ se, e só se, $A=\mathbb{N}$.

Note que não existe $A \in \mathcal{A}$ tal que $\operatorname{ppc}(A)=1$.

Lema 2.2.3. Seja $A \in \mathcal{A}$. Se $B \supseteq A$ e $o(A)=o(B)$, então $\operatorname{ppc}(B) \geq \operatorname{ppc}(A)$.

Demonstração: $\operatorname{Se} \operatorname{ppc}(B)<\operatorname{ppc}(A)$, então

$$
o(A)=o(B)=o(\operatorname{ppc}(B), B) \leq o(\operatorname{ppc}(B), A)<o(A),
$$

absurdo. 
Proposição 2.2.4. O único ponto isolado de $\mathcal{A}$ é $\mathbb{N}$.

Demonstração: $\mathbb{N}$ é um ponto isolado de $\mathcal{A}$ porque

$$
\mathcal{B}(\mathbb{N}, 1)=\{\mathbb{N}\}
$$

De fato, se $d(A, \mathbb{N})<1$, então

$$
o(A)=o(\mathbb{N})=1
$$

o que implica $A=\mathbb{N}$. Agora seja $A \in \mathcal{A}, A \neq \mathbb{N}$. Então $o(A) \geq 2$. Seja $\varepsilon>0$. Mostraremos que $A$ é um ponto de acumulação de $\mathcal{A}$. Para isso, seja $n_{0} \in \mathbb{N}$ tal que

$$
\sum_{\alpha>n_{0}} \frac{1}{\alpha^{2}}<\varepsilon
$$

Se $\mathbb{N}-A$ for infinito, seja

$$
n_{1}=\max \left\{n_{0}, \operatorname{ppc}(A)\right\} .
$$

Como $\mathbb{N}-A$ é infinito, existe $x \in \mathbb{N}-A$ tal que $x>n_{1}$. Então

$$
B=A \cup\{x\}
$$

é tal que $B \neq A$ e $o(B)=o(A)$, pois $x>n_{1} \geq \operatorname{ppc}(A)$. Além disso,

$$
d(A, B)=\frac{1}{x^{2}}<\sum_{\alpha>n_{1}} \frac{1}{\alpha^{2}} \leq \sum_{\alpha>n_{0}} \frac{1}{\alpha^{2}}<\varepsilon .
$$

Se $\mathbb{N}-A$ for finito, seja $n_{2} \in \mathbb{N}$ tal que se $n \geq n_{2}$, então $n \in A$. Se

$$
x=\max \left\{n_{0}, n_{2}, \operatorname{ppc}(A)\right\},
$$

então

$$
B=A-\{x+1\}
$$

é tal que $B \neq A$ e $o(B)=o(A)$, pois $x \in A$ e, portanto, $o(x+1, B)=2 \leq o(A)$. Logo

$$
d(A, B)=\frac{1}{(x+1)^{2}}<\sum_{\alpha>x} \frac{1}{\alpha^{2}} \leq \sum_{\alpha>n_{0}} \frac{1}{\alpha^{2}}<\varepsilon .
$$

Proposição 2.2.5. O conjunto

$$
E=\{A \in \mathcal{A} ; \mathbb{N}-A \text { é finito }\}
$$

é enumerável e denso em $\mathcal{A}$. Em particular, $\mathcal{A}$ é um espaço métrico separável.

Demonstração: É claro que $E$ é enumerável. Vamos mostrar que $E$ é denso em $\mathcal{A}$. Para isto, sejam $A \in \mathcal{A}$ e $\varepsilon>0$, e seja $n_{0} \in \mathbb{N}$ tal que

$$
\sum_{\alpha>n_{0}} \frac{1}{\alpha^{2}}<\varepsilon
$$


Seja

$$
x=\max \left\{n_{0}, \operatorname{ppc}(A)\right\}+1 .
$$

Se

$$
B=A \cup\{y \in \mathbb{N} ; y \geq x\},
$$

então $B \in E, o(B)=o(A)(\operatorname{pois} x>\operatorname{ppc}(A))$, e

$$
d(A, B)=\sum_{\alpha \in A \triangle B} \frac{1}{\alpha^{2}} \leq \sum_{\alpha \geq x}^{\infty} \frac{1}{\alpha^{2}} \leq \sum_{\alpha>n_{0}}^{\infty} \frac{1}{\alpha^{2}}<\varepsilon .
$$

Logo $E$ é denso em $\mathcal{A}$.

Proposição 2.2.6. A função

$$
\begin{aligned}
\operatorname{ppc}: & \mathcal{A} \\
A & \mapsto \operatorname{ppc}(A)
\end{aligned}
$$

é contínua em $\mathcal{A}$, mas não é uniformemente contínua em $\mathcal{A}$.

Demonstração: Seja $A \in \mathcal{A}, A \neq \mathbb{N}$ para evitar o caso trivial. Dado $\varepsilon>0$, seja

$$
\delta=\frac{1}{\operatorname{ppc}(A)^{2}}>0 \text {. }
$$

Se

$$
d(A, B)<\delta \leq 1
$$

então $o(A)=o(B) \mathrm{e}$

$$
A \cap \overleftarrow{\operatorname{ppc}(A)}=B \cap \overleftarrow{\operatorname{ppc}(A)}
$$

Isto implica $\operatorname{ppc}(A)=\operatorname{ppc}(B)$, ou seja $|\operatorname{ppc}(A)-\operatorname{ppc}(B)|=0<\varepsilon$. Logo ppc é contínua em $\mathcal{A}$.

Se ppc fosse uniformemente contínua em $\mathcal{A}$, dado

$$
\varepsilon=\frac{1}{2}
$$

existiria $\delta>0$ tal que se $d(A, B)<\delta$, então

$$
|\operatorname{ppc}(A)-\operatorname{ppc}(B)|<\frac{1}{2}, \forall A, B \in \mathcal{A} .
$$

Em particular, isto implicaria $\operatorname{ppc}(A)=\operatorname{ppc}(B), \forall A, B \in \mathcal{A}$ tais que $d(A, B)<\delta$. Seja $x \in \mathbb{N}-\{0,1\}$ tal que

$$
\frac{1}{x^{2}}+\frac{1}{(x+1)^{2}}<\delta
$$

Se $A=\mathbb{N}-\{x\}$ e $B=\mathbb{N}-\{x+1\}$, então $o(A)=o(B)=2$,

$$
d(A, B)=\frac{1}{x^{2}}+\frac{1}{(x+1)^{2}}<\delta
$$

$\operatorname{e} \operatorname{ppc}(A)=x \neq x+1=\operatorname{ppc}(B)$, absurdo. 
Corolário 2.2.7. Seja $X \subseteq \mathcal{A}$. Se existir $M>0$ tal que

$$
\operatorname{ppc}(A) \leq M, \forall A \in X,
$$

então $\operatorname{ppc}(A) \leq M, \forall A \in \bar{X}$.

Demonstração: Seja $A \in \bar{X}$. Então existe $\left\{A_{n}\right\}_{n \in \mathbb{N}} \subseteq X$ tal que $A_{n} \rightarrow A$. Como a função ppc é contínua em $\mathcal{A}$,

$$
\operatorname{ppc}\left(A_{n}\right) \rightarrow \operatorname{ppc}(A)
$$

Como todos os pontos de $\mathbb{N}$ são isolados, existe $n_{0} \in \mathbb{N}$ tal que

$$
\operatorname{ppc}\left(A_{n}\right)=\operatorname{ppc}(A), \forall n \geq n_{0} .
$$

Logo $\operatorname{ppc}(A)=\operatorname{ppc}\left(A_{n_{0}}\right) \leq M$.

Definamos agora o conceito de pseudoconvergência para sequências em $\mathcal{A}$.

Definição 2.2.8. Seja $\left\{A_{n}\right\}_{n \in \mathbb{N}}$ uma sequência em $\mathcal{A}$. Dizemos que $\left\{A_{n}\right\}_{n \in \mathbb{N}}$ pseudoconverge para $A \in \mathcal{A}$ se dado $\varepsilon>0$ existe $n_{0} \in \mathbb{N}$ tal que se $n \geq n_{0}$, então

$$
\sum_{\alpha \in A_{n} \triangle A} \frac{1}{\alpha^{2}}<\varepsilon .
$$

Denotamos isto por $A_{n} \stackrel{p}{\rightarrow} A$.

Segue diretamente do lema 2.1.4 que se $\left\{A_{n}\right\}_{n \in \mathbb{N}}$ for uma sequência em $\mathcal{A}$ tal que

$$
A_{n} \stackrel{p}{\rightarrow} A \in \mathcal{A}
$$

então

$$
A=\bigcup_{j=0}^{\infty} \bigcap_{m=j}^{\infty} A_{m}
$$

Em particular, o limite de uma pseudoconvergência, quando existe, é único. Deixemos isto registrado.

Proposição 2.2.9. Seja $\left\{A_{n}\right\}_{n \in \mathbb{N}}$ uma sequência em $\mathcal{A}$. Se $A_{n} \rightarrow A \in \mathcal{A}$, então $A_{n} \stackrel{p}{\rightarrow}$ A. Em particular,

$$
A=\bigcup_{j=1}^{\infty} \bigcap_{m=j}^{\infty} A_{m}
$$

Demonstração: Se $A_{n} \rightarrow A$, dado $\varepsilon>0$ existe $n_{0} \in \mathbb{N}$ tal que se $n \geq n_{0}$, então $d\left(A, A_{n}\right)<\varepsilon$. Assim, se $n \geq n_{0}$, então

$$
\sum_{\alpha \in A_{n} \triangle A} \frac{1}{\alpha^{2}} \leq d\left(A_{n}, A\right)<\varepsilon .
$$

$\operatorname{Logo} A_{n} \stackrel{p}{\rightarrow} A$. 
A recíproca da proposição anterior é falsa. De fato, considere a sequência de bases aditivas

$$
A_{n}=\mathbb{N}-\{n\}, \forall n \in \mathbb{N}-\{0,1\} .
$$

É claro que $\left\{A_{n}\right\}_{n \in \mathbb{N}-\{0,1\}}$ diverge. Entretanto, $A_{n} \stackrel{p}{\rightarrow} \mathbb{N}$. Com efeito, dado $\varepsilon>0$, seja $n_{0} \in \mathbb{N}-\{0,1\}$ tal que

$$
\frac{1}{n_{0}^{2}}<\varepsilon
$$

Assim, se $n \geq n_{0}$, então

$$
\sum_{\alpha \in A_{n} \triangle \mathbb{N}} \frac{1}{\alpha^{2}}=\frac{1}{n^{2}} \leq \frac{1}{n_{0}^{2}}<\varepsilon \Rightarrow A_{n} \stackrel{p}{\rightarrow} \mathbb{N} .
$$

Lema 2.2.10. Seja $\left\{A_{n}\right\}_{n \in \mathbb{N}}$ uma sequência em $\mathcal{A}$. Se $A_{n} \stackrel{p}{\rightarrow} A \in \mathcal{A}$, então existe $n_{0} \in \mathbb{N}$ tal que $o\left(A_{n}\right) \geq o(A), \forall n \geq n_{0}$.

Demonstração: Seja $x=\operatorname{ppc}(A)+1$. Como $A_{n} \stackrel{p}{\rightarrow} A$, dado

$$
\varepsilon=\frac{1}{x^{2}}
$$

existe $n_{0} \in \mathbb{N}$ tal que se $n \geq n_{0}$, então

$$
\sum_{\alpha \in A_{n} \triangle A} \frac{1}{\alpha^{2}}<\frac{1}{x^{2}} .
$$

Em particular,

$$
A_{n} \cap \overleftarrow{x}=A \cap \overleftarrow{x}, \forall n \geq n_{0}
$$

Assim, se $n \geq n_{0}$, então

$$
o\left(\operatorname{ppc}(A), A_{n}\right)=o(\operatorname{ppc}(A), A)=o(A),
$$

o que implica $o\left(A_{n}\right) \geq o(A), \forall n \geq n_{0}$.

Teorema 2.2.11. Seja $\left\{A_{n}\right\}_{n \in \mathbb{N}}$ uma sequência em $\mathcal{A}$. Se $A_{n} \stackrel{p}{\rightarrow} A \in \mathcal{A}$ e se existir $M>0$ tal que $\operatorname{ppc}\left(A_{n}\right) \leq M, \forall n \in \mathbb{N}$, então $A_{n} \rightarrow A$.

Demonstração: Afirmo que é suficiente mostrar que existe $n_{1} \in \mathbb{N}$ tal que se $n \geq n_{1}$, então $o(A)=o\left(A_{n}\right)$. De fato, mostrado isto, como $A_{n} \stackrel{p}{\rightarrow} A$, então dado $\varepsilon>0$ existe $n_{0} \in \mathbb{N}$ tal que se $n \geq n_{0}$, então

$$
\sum_{\alpha \in A_{n} \triangle A} \frac{1}{\alpha^{2}}<\varepsilon .
$$

Se $n_{2}=\max \left\{n_{0}, n_{1}\right\}$ e se $n \geq n_{2}$, então

$$
d\left(A_{n}, A\right)=\sum_{\alpha \in A_{n} \triangle A} \frac{1}{\alpha^{2}}<\varepsilon,
$$

o que implica $A_{n} \rightarrow A$. 
Pelo lema anterior, existe $n_{3} \in \mathbb{N}$ tal que se $n \geq n_{3}$, então $o\left(A_{n}\right) \geq o(A)$. Suponha por absurdo que não exista $n_{1} \in \mathbb{N}$ tal que se $n \geq n_{1}$, então $o(A)=o\left(A_{n}\right)$. Então existirá uma subsequência $\left\{A_{m}\right\}_{m \in \mathbb{N}}$ de $\left\{A_{n}\right\}_{n \in \mathbb{N}}$ tal que

$$
o\left(A_{m}\right)>o(A), \forall m \in \mathbb{N} .
$$

Agora seja $y \in \mathbb{Z}_{+}$. Como $A_{n} \stackrel{p}{\rightarrow} A$, dado

$$
\varepsilon=\frac{1}{y^{2}}
$$

existe $n_{4} \in \mathbb{N}$ tal que se $n \geq n_{4}$, então

$$
\sum_{\alpha \in A_{n} \triangle A} \frac{1}{\alpha^{2}}<\frac{1}{y^{2}} .
$$

Em particular,

$$
A_{n} \cap \overleftarrow{y}=A \cap \overleftarrow{y}, \forall n \geq n_{4}
$$

Se $m \in \mathbb{N}$ for tal que $m \geq n_{4}$, então

$$
A \cap \overleftarrow{y}=A_{m} \cap \overleftarrow{y}
$$

Se $z \in \mathbb{N}$ e $z \leq y$, então $o\left(z, A_{m}\right)=o(z, A) \leq o(A)$. Como $o\left(A_{m}\right)>o(A)$, então $\operatorname{ppc}\left(A_{m}\right)>y$. Como $y$ é arbitrário, não existe $M>0$ tal que

$$
\operatorname{ppc}\left(A_{n}\right) \leq M, \forall n \in \mathbb{N},
$$

absurdo.

Teorema 2.2.12. Toda sequência de Cauchy em $\mathcal{A}$ pseudoconverge. Mais especificamente, se $\left\{A_{n}\right\}_{n \in \mathbb{N}}$ for uma sequência de Cauchy em $\mathcal{A} e$

$$
A=\bigcup_{m=0}^{\infty} \bigcap_{n=m}^{\infty} A_{n},
$$

então $A \in \mathcal{A}, A_{n} \stackrel{p}{\rightarrow} A$ e $o(A) \leq \lim _{n \rightarrow \infty} o\left(A_{n}\right)$.

Demonstração: Como $\left\{A_{n}\right\}_{n \in \mathbb{N}}$ é uma sequência de Cauchy, dado

$$
\varepsilon=\frac{1}{2}
$$

existe $n_{0} \in \mathbb{N}$ tal que se $n, m \geq n_{0}$, então

$$
d\left(A_{n}, A_{m}\right)<\frac{1}{2}
$$

Em particular,

$$
o\left(A_{n}\right)=o\left(A_{m}\right), \forall n, m \geq n_{0} .
$$

Assim existe $\lim _{n \rightarrow \infty} o\left(A_{n}\right)$. Seja

$$
L=\lim _{n \rightarrow \infty} o\left(A_{n}\right) .
$$


Para mostrar que $A \in \mathcal{A}$ é suficiente mostrar que

$$
o(x, A) \leq L, \forall x \in \mathbb{N} .
$$

Para isto, seja $x \in \mathbb{N}, x \geq 2$, uma vez que $\{0,1\} \subseteq A$. Como $\left\{A_{n}\right\}_{n \in \mathbb{N}}$ é uma sequência de Cauchy, dado

$$
\varepsilon=\frac{1}{x^{2}}
$$

existe $n_{1} \in \mathbb{N}$ tal que se $n, m \geq n_{1}$, então

$$
d\left(A_{n}, A_{m}\right)<\frac{1}{x^{2}}
$$

Em particular,

$$
A_{n} \cap \overleftarrow{x}=A_{m} \cap \overleftarrow{x}, \forall n, m \geq n_{1}
$$

Seja $n_{2}=\max \left\{n_{0}, n_{1}\right\}$. Afirmo que

$$
A_{n_{2}} \cap \overleftarrow{x}=A \cap \overleftarrow{x}
$$

De fato:

$(\subseteq)$ Seja $y \in A_{n_{2}}, y \leq x$. Sabemos que

$$
\bigcap_{m=n_{2}}^{\infty} A_{m} \subseteq A .
$$

Como $n_{2} \geq n_{1}$, então

$$
A_{n_{2}} \cap \overleftarrow{x}=A_{m} \cap \overleftarrow{x}, \forall m \geq n_{2}
$$

Em particular, $y \in A_{m}, \forall m \geq n_{2}$, o que implica $y \in A$.

() Seja $y \in A, y \leq x$. Como $y \in A$, existe $m \in \mathbb{N}$ tal que

$$
y \in \bigcap_{n=m}^{\infty} A_{n} .
$$

Seja $n_{3}=\max \left\{n_{2}, m\right\}$. Como $n_{3} \geq m$, então $y \in A_{n_{3}}$. Como $n_{3} \geq n_{2}$ e $y \leq x$, então $y \in A_{n_{2}}$. Portanto

$$
o(x, A)=o\left(x, A_{n_{2}}\right) \leq o\left(A_{n_{2}}\right)=L .
$$

Em particular, $A \in \mathcal{A}$ e $o(A) \leq L$.

Agora vamos mostrar que $A_{n} \stackrel{p}{\rightarrow} A$. Para isto, seja $\varepsilon>0$ e seja $z \in \mathbb{Z}_{+}$tal que

$$
\sum_{\alpha>z} \frac{1}{\alpha^{2}}<\varepsilon
$$

Como $\left\{A_{n}\right\}_{n \in \mathbb{N}}$ é uma sequência de Cauchy, dado

$$
\varepsilon^{\prime}=\frac{1}{z^{2}}
$$


existe $n_{4} \in \mathbb{N}$ tal que se $n, m \geq n_{4}$, então

$$
d\left(A_{n}, A_{m}\right)<\frac{1}{z^{2}} .
$$

Em particular,

$$
A_{n} \cap \overleftarrow{z}=A_{m} \cap \overleftarrow{z}, \forall n, m \geq n_{4}
$$

Da mesma forma que antes,

$$
A_{n_{4}} \cap \overleftarrow{z}=A \cap \overleftarrow{z}
$$

Isto implica

$$
A \cap \overleftarrow{z}=A_{n} \cap \overleftarrow{z}, \forall n \geq n_{4}
$$

Assim, se $n \geq n_{4}$, então

$$
\sum_{\alpha \in A_{n} \triangle A} \frac{1}{\alpha^{2}} \leq \sum_{\alpha>z} \frac{1}{\alpha^{2}}<\varepsilon .
$$

$\operatorname{Logo} A_{n} \stackrel{p}{\rightarrow} A$.

A recíproca do teorema anterior não vale. Por exemplo, a sequência $\left\{A_{n}\right\}_{n \in \mathbb{Z}_{+}}$ dada por

$$
A_{n}=\left\{0,1, \ldots, n, n^{2}+1, \ldots\right\}
$$

é tal que $A_{n} \stackrel{p}{\rightarrow} \mathbb{N}$, pois

$$
\sum_{\alpha \in A_{n} \triangle \mathbb{N}} \frac{1}{\alpha^{2}}=\sum_{\alpha=n+1}^{n^{2}} \frac{1}{\alpha^{2}} \leq \int_{n}^{n^{2}} \frac{1}{x^{2}} d x \underset{n \rightarrow \infty}{\longrightarrow} 0,
$$

$\operatorname{mas}\left\{A_{n}\right\}_{n \in \mathbb{Z}_{+}}$não é uma sequência de Cauchy, pois

$$
o\left(A_{n}\right)=n, \forall n \in \mathbb{Z}_{+} .
$$

O lema abaixo permitir-nos-á dar uma caracterização simples dos subconjuntos compactos de $\mathcal{A}$ :

Lema 2.2.13. Seja $X \subseteq \mathcal{A}$. Se existir $M>0$ tal que $\operatorname{ppc}(A) \leq M, \forall A \in X$, então $\bar{X}$ será completo.

Demonstração: Seja $\left\{A_{n}\right\}_{n \in \mathbb{N}}$ uma sequência de Cauchy em $\bar{X}$. Pelo teorema 2.2.12, existe $A \in \mathcal{A}$ tal que $A_{n} \stackrel{p}{\rightarrow} A$. Como existe $M>0$ tal que

$$
\operatorname{ppc}(A) \leq M, \forall A \in X,
$$

o corolário 2.2.7 implica que

$$
\operatorname{ppc}\left(A_{n}\right) \leq M, \forall n \in \mathbb{N} .
$$

Pelo teorema 2.2.11,

$$
A_{n} \rightarrow A
$$

Como $\bar{X}$ é fechado, $A \in \bar{X}$. Então $\bar{X}$ é completo. 
A recíproca do lema anterior não vale. De fato, considere a sequência

$$
A_{n}=\mathbb{N}-\{2, \ldots, n\}, n \geq 2
$$

e seja $X=\left\{A_{n}\right\}_{n \geq 2}$. Então

$$
o\left(A_{n}\right)=n=\operatorname{ppc}\left(A_{n}\right)
$$

Segue que $X$ é completo, pois $d\left(A_{n}, A_{m}\right)>1$ se $n \neq m$, mas não existe $M>0$ tal que $\operatorname{ppc}(A) \leq M, \forall A \in X$.

Note também que nós não podemos concluir que $X$ é completo no lema 2.2.13. De fato, seja

$$
A_{n}=\mathbb{N}-\{2, n\}, n \geq 2,
$$

e seja $X=\left\{A_{n}\right\}_{n \geq 2}$. Então

$$
A_{n} \rightarrow \mathbb{N}-\{2\} \notin X
$$

Claramente, o que acontece aqui é que a base aditiva $A$, limite da sequência $\left\{A_{n}\right\}_{n \geq 2}$, pode não pertencer a $X$.

Teorema 2.2.14 (Teorema da caracterização dos subconjuntos compactos de $\mathcal{A}$ ). Seja $X \subseteq \mathcal{A}$. As seguintes afirmações são equivalentes.

1. X é compacto.

2. $X$ é fechado, limitado, e existe $M>0$ tal que $\operatorname{ppc}(A) \leq M, \forall A \in X$.

Demonstração: (1. $\Rightarrow$ 2.) Se $X$ for compacto, claramente $X$ deve ser fechado e limitado. Como a função ppc é contínua em $\mathcal{A}$, o conjunto

$$
\operatorname{ppc}(X)=\{\operatorname{ppc}(A) ; A \in X\}
$$

é compacto em $\mathbb{N}$. Em particular, existe $M>0$ tal que $\operatorname{ppc}(A) \leq M, \forall A \in X$.

(2. $\Rightarrow$ 1.) Como $X$ limitado, o corolário 2.1.6 implica que $X$ é totalmente limitado. Como existe $M>0$ tal que

$$
\operatorname{ppc}(A) \leq M, \forall A \in X,
$$

segue do lema 2.2.13 que $\bar{X}$ é completo. Mas então $X=\bar{X}$, o que implica $X$ é completo. Logo $X$ é compacto.

Corolário 2.2.15. Seja $A \in \mathcal{A}, A \neq \mathbb{N}$. Se

$$
0<r \leq \frac{1}{\operatorname{ppc}(A)^{2}}
$$

então $\mathcal{B}[A, r]$ é um subconjunto compacto de $\mathcal{A}$. Em particular, $\mathcal{A}$ é um espaço métrico localmente compacto. 
Demonstração: É suficiente mostrar que

$$
\mathcal{B}\left[A, \frac{1}{\operatorname{ppc}(A)^{2}}\right]
$$

é um subconjunto compacto de $\mathcal{A}$. Como

$$
\mathcal{B}\left[A, \frac{1}{\operatorname{ppc}(A)^{2}}\right]
$$

é um subconjunto limitado e fechado de $\mathcal{A}$, pelo teorema anterior é suficiente mostrar que existe $M>0$ tal que

$$
\operatorname{ppc}(A) \leq M, \forall A \in \mathcal{B}\left[A, \frac{1}{\operatorname{ppc}(A)^{2}}\right] .
$$

Pelo corolário 2.2.7, é suficiente mostrar que existe $M>0$ tal que

$$
\operatorname{ppc}(A) \leq M, \forall A \in \mathcal{B}\left(A, \frac{1}{\operatorname{ppc}(A)^{2}}\right)
$$

Para isto, seja

$$
B \in \mathcal{B}\left(A, \frac{1}{\operatorname{ppc}(A)^{2}}\right)
$$

Então

$$
d(A, B)<\frac{1}{\operatorname{ppc}(A)^{2}} \leq \frac{1}{4} .
$$

Em particular, $o(A)=o(B) \mathrm{e}$

$$
A \cap \overleftarrow{\mathrm{ppc}(A)}=B \cap \overleftarrow{\mathrm{ppc}(A)}
$$

o que implica $\operatorname{ppc}(A)=\operatorname{ppc}(B)$.

Note que, no espaço $(\mathcal{A}, d)$, bolas pequenas são compactas. Note também que bolas abertas também podem ser compactas, bastando para isto uma pequena condição extra. De fato, seja $A \in \mathcal{A}, A \neq \mathbb{N}$. Se

$$
0<r \leq \frac{1}{\operatorname{ppc}(A)^{2}}
$$

e $\mathcal{S}(A, r)=\emptyset$, então $\mathcal{B}(A, r)$ é um subconjunto compacto de $\mathcal{A}$, uma vez que

$$
\mathcal{B}(A, r)=\mathcal{B}[A, r]-\mathcal{S}(A, r) .
$$

A próxima seção deste capítulo se encarregará de dar uma caracterização topológica de $(\mathcal{A}, d)$. 


\subsection{Caracterização topológica de $(\mathcal{A}, d)$}

Com o intuito de caracterizar completamente o espaço métrico $(\mathcal{A}, d)$, vamos particioná-lo de uma maneira bastante natural. Para isto, se $h, m \in \mathbb{N}$, denotaremos

$$
\mathcal{A}_{h}^{m}=\{A \in \mathcal{A} ; o(A)=h, \operatorname{ppc}(A)=m\} .
$$

Proposição 2.3.1. Sejam $h, m \in \mathbb{Z}_{+}$. Então cada $\mathcal{A}_{h}^{m}$ é um subconjunto compacto e aberto de $\mathcal{A}$,

$$
\mathcal{A}=\bigcup_{h, m \in \mathbb{N}} \mathcal{A}_{h}^{m}
$$

e os conjuntos $\mathcal{A}_{h}^{m}$ são dois a dois disjuntos.

Demonstração: Sabemos que as funções $o: \mathcal{A} \rightarrow \mathbb{Z}_{+}$e ppc $: \mathcal{A} \rightarrow \mathbb{N}$ são contínuas. Como

$$
\mathcal{A}_{h}^{m}=o^{-1}(\{h\}) \cap \operatorname{ppc}^{-1}(\{m\}),
$$

então cada $\mathcal{A}_{h}^{m}$ é aberto em $\mathcal{A}$. A compacidade segue diretamente do teorema 2.2.14 e as outras afirmações são óbvias.

Vamos agora recordar um conceito topológico que será útil na caracterização topológica de $(\mathcal{A}, d)$.

Definição 2.3.2. Um espaço topológico $(T, \tau)$ é zero dimensional se ele possui uma base de subconjuntos que são ao mesmo tempo abertos e fechados.

Teorema 2.3.3. Seja $(T, \tau)$ um espaço topológico. Se $(T, \tau)$ for zero dimensional e $\mathrm{T}_{1}$, então $(T, \tau)$ será totalmente separado e Hausdorff. Se $(T, \tau)$ for totalmente desconexo, Hausdorff e localmente compacto, então $(T, \tau)$ será zero dimensional.

A primeira afirmação do teorema acima é trivial, enquanto que para uma prova da segunda afirmação recomendamos [9], p. 136.

Corolário 2.3.4. $(\mathcal{A}, d)$ é um espaço métrico zero dimensional.

Agora vamos proceder à caracterização topológica de $(\mathcal{A}, d)$. Para isto, invocamos o teorema da caracterização topológica do conjunto de Cantor de Brouwer.

Teorema 2.3.5 (Brouwer, 1910). Todo espaço métrico compacto zero dimensional sem pontos isolados é homeomorfo ao conjunto de Cantor $\mathcal{C}$.

Recomendamos [86] para uma prova deste teorema.

Teorema 2.3.6. Sejam $h, m \in \mathbb{Z}, h, m \geq 2$. Então $\mathcal{A}_{h}^{m}$ é homeomorfo ao conjunto de Cantor $\mathcal{C}$.

Demonstração: Sejam $h, m \in \mathbb{Z}, h, m \geq 2$. Então $\mathcal{A}_{h}^{m}$ é compacto pelo teorema 2.2.14. Como todo subespaço de um espaço zero dimensional é zero dimensional, $\mathcal{A}_{h}^{m}$ é zero dimensional. E como a mesma prova da proposição 2.2.4 serve para mostrar que $\mathcal{A}_{h}^{m}$ não tem ponto isolado, segue do teorema 2.3 .5 que $\mathcal{A}_{h}^{m}$ é homeomorfo ao conjunto de Cantor $\mathcal{C}$. 
Lema 2.3.7. Sejam $h, h^{\prime}, m, m^{\prime} \in \mathbb{Z}$ tais que $h, h^{\prime}, m, m^{\prime} \geq 2$. Se $A \in \mathcal{A}_{h}^{m}, B \in \mathcal{A}_{h}^{m^{\prime}}$ e $m<m^{\prime}$, então

$$
\min (A \triangle B) \leq m
$$

Em particular, $d\left(\mathcal{A}_{h}^{m}, \mathcal{A}_{h^{\prime}}^{m^{\prime}}\right)>0$ se $(h, m) \neq\left(h^{\prime}, m^{\prime}\right)$.

Demonstração: Suponha por absurdo que $\min (A \triangle B)>m$. Então $A \cap \overleftarrow{m}=B \cap \overleftarrow{m}$ o que implica

$$
o(m, B)=o(m, A)=o(\operatorname{ppc}(A), A)=o(A)=o(B),
$$

o que, por sua vez, implica $m^{\prime}=\operatorname{ppc}(B) \leq m$, absurdo.

Para a parte final do lema, se $h \neq h^{\prime}$, então $d\left(\mathcal{A}_{h}^{m}, \mathcal{A}_{h^{\prime}}^{m^{\prime}}\right) \geq 1$. Se $h=h^{\prime}$, $m<m^{\prime}, A \in \mathcal{A}_{h}^{m}$ e $B \in \mathcal{A}_{h}^{m^{\prime}}$, então

$$
d(A, B) \geq \frac{1}{(\min A \triangle B)^{2}} \geq \frac{1}{m^{2}},
$$

o que implica

$$
d\left(\mathcal{A}_{h}^{m}, \mathcal{A}_{h^{\prime}}^{m^{\prime}}\right) \geq \frac{1}{m^{2}}>0
$$

Teorema 2.3.8 (Teorema da caracterização topológica de $(\mathcal{A}, d)$ ). O espaço métrico $(\mathcal{A}, d)$ é homeomorfo à união disjunta (topológica) de um ponto com a união disjunta (topológica) de uma quantidade enumerável de conjuntos de Cantor.

Demonstração: Basta relacionar $\mathcal{A}_{1}^{0}=\{\mathbb{N}\}$ ao ponto isolado do espaço do enunciado do teorema e cada um $\operatorname{dos} \mathcal{A}_{h}^{m}, h, m \in \mathbb{Z}, h, m \geq 2$, a um conjunto de Cantor. Como

$$
d\left(\mathcal{A}_{h}^{m}, \mathcal{A}_{h^{\prime}}^{m^{\prime}}\right)>0
$$

se $(h, m) \neq\left(h^{\prime}, m^{\prime}\right)$, o teorema está demonstrado. 


\section{Capítulo 3}

\section{Bases aditivas finas e aplicações}

Este capítulo objetiva mostrar que o conjunto das bases finas é denso em $(\mathcal{A}, d)$, assim como exibir uma classe de funções

$$
f: \mathcal{A} \rightarrow \mathcal{A}
$$

que têm propriedades muito boas com relação à função ordem. Em particular, será apresentado um critério para saber quando podemos acrescentar um determinado conjunto de números a uma base especificada sem alterar sua ordem. Isso é particularmente interessante para abordar problemas como a conjectura de Goldbach. Voltaremos a falar disso na terceira seção deste capítulo. Por hora, vamos iniciar este capítulo desenvolvendo um teorema que funciona como um binômio de Newton para bases aditivas em geral.

\subsection{Um binômio de Newton para bases aditivas}

Adicionar elementos em uma base aditiva é um processo difícil de entender. De fato, pode-se adicionar um único número a uma dada base e diminuir sua ordem (coloque o 2 em $\mathbb{N}-\{2\}$ ), ou pode-se adicionar uma quantidade infinita de números em outra base e não alterar sua ordem (coloque $\{5,7,9,11,13,15, \ldots\}$ em $\{0,1,2,4,6,8,10, \ldots\})$. Este problema é equivalente ao problema de tirar um número $n \geq 2$ de uma dada base $A \in \mathcal{A}$ e procurar saber o que acontece com a ordem de $A$, e isto tem relação tanto com o teorema de Mann (teorema 1.3.4) quanto com seu corolário (corolário 1.3.5). Sobre este assunto, apresentamos a seguinte proposição.

Proposição 3.1.1. Seja $A \in \mathcal{A}$. Se $a \in A-\{0,1\}$, então $A-\{a\} \in \mathcal{A} e$

$$
o(A-\{a\}) \leq a \cdot o(A) \text {. }
$$

Demonstração: É suficiente mostrar que

$$
o(n, A-\{a\}) \leq a \cdot o(A), \forall n \geq 2 .
$$


Para isto, fixe $n \geq 2$. Como $A \in \mathcal{A}$, existem $a_{1}, \ldots, a_{o(A)} \in A$ tais que

$$
n=a_{1}+\cdots a_{o(A)} .
$$

Vamos dividir a demonstração em três casos.

$1^{\mathrm{o}}$ caso: $a_{i}=a, \forall i \in \overleftarrow{o(A)}$. Neste caso,

$$
n=\underbrace{a+\cdots+a}_{o(A) \text { parcelas }}=a \cdot o(A)=\underbrace{1+\cdots+1}_{a \cdot o(A) \text { parcelas }}
$$

o que implica $o(n, A-\{a\}) \leq a \cdot o(A)$.

$2^{\circ}$ caso: $a_{i} \neq a, \forall i \in \overleftarrow{o(A)}$. Neste caso,

$$
o(n, A-\{a\})=o(n, A) \leq o(A)<a \cdot o(A) .
$$

$3^{\circ}$ caso: existe $j \in \overleftarrow{o(A)-1}$ tal que

$$
\left\{\begin{array}{c}
a_{1}=\cdots=a_{j}=a \\
a_{j+1}, \ldots, a_{o(A)} \neq a
\end{array}\right.
$$

Então

$$
n=j a+a_{j+1}+\cdots+a_{o(A)}=\underbrace{1+\cdots+1}_{j a \text { parcelas }}+\underbrace{a_{j+1}}_{\neq a}+\cdots+\underbrace{a_{o(A)}}_{\neq a},
$$

o que implica $o(n, A-\{a\}) \leq j a+o(A)-j<a \cdot o(A)$.

Note que a cota superior obtida na proposição anterior não pode ser melhorada: $o(\mathbb{N})=1$ e $o(\mathbb{N}-\{2\})=2=2 \cdot o(\mathbb{N})$. Basicamente, o que ocorre aqui é que, salvo o fato de termos mais informações a respeito de uma base aditiva $A$, um estudo geral sobre todas as bases aditivas obriga a cota trivial ser a melhor cota que pode ser obtida.

Para desenvolver o "binômio de Newton" para as bases aditivas, vamos começar com dois lemas.

Lema 3.1.2. Sejam $h_{1}, h_{2} \in \mathbb{Z}_{+} e A \subseteq \mathbb{N}$. Então $\left(h_{1}+h_{2}\right) A=h_{1} A+h_{2} A$.

Demonstração: Temos $\left(h_{1}+h_{2}\right) A=$

$$
\begin{aligned}
& =\left\{a_{1}+\cdots+a_{h_{1}+h_{2}} ; a_{1}, \ldots, a_{h_{1}+h_{2}} \in A\right\} \\
& =\left\{a_{1}^{\prime}+\cdots+a_{h_{1}}^{\prime} ; a_{1}^{\prime}, \ldots, a_{h_{1}}^{\prime} \in A\right\}+\left\{a_{1}^{\prime \prime}+\cdots+a_{h_{2}}^{\prime \prime} ; a_{1}^{\prime \prime}, \ldots, a_{h_{2}}^{\prime \prime} \in A\right\} \\
& =h_{1} A+h_{2} A .
\end{aligned}
$$

Lema 3.1.3. Se $\{0,1\} \subseteq A_{1}, A_{2}, A_{3} \subseteq \mathbb{N}$, então

$$
\left(A_{1} \cup A_{2}\right)+A_{3}=\left(A_{1}+A_{3}\right) \cup\left(A_{2}+A_{3}\right) .
$$


Demonstração: Se $x \in\left(A_{1} \cup A_{2}\right)+A_{3}$, então $x=a+a_{3}$, onde $a \in A_{1} \cup A_{2}$ e $a_{3} \in A_{3}$. Se $a \in A_{1}$, então $x \in A_{1}+A_{3}$. Se $a \in A_{2}$, então $x \in A_{2}+A_{3}$. De qualquer forma, $x \in\left(A_{1}+A_{3}\right) \cup\left(A_{2}+A_{3}\right)$.

Agora seja $x \in\left(A_{1}+A_{3}\right) \cup\left(A_{2}+A_{3}\right)$. Suponha sem perda de generalidade que $x \in A_{1}+A_{3}$. Então $x=a_{1}+a_{3}$, onde $a_{1} \in A_{1}$ e $a_{3} \in A_{3}$. Então $a \in A_{1} \cup A_{2}$, o que implica $x \in\left(A_{1} \cup A_{2}\right)+A_{3}$.

Corolário 3.1.4. Se $\{0,1\} \subseteq A_{1}, \ldots, A_{h}, B \subseteq \mathbb{N}$, então

$$
\left(\bigcup_{i=1}^{h} A_{i}\right)+B=\bigcup_{i=1}^{h}\left(A_{i}+B\right)
$$

Demonstração: Basta aplicar indução no lema anterior.

Se $\{0,1\} \subseteq A \subseteq \mathbb{N}$, denotaremos $0 A=\{0\}$, para simplificar a notação.

Teorema 3.1.5 (Binômio de Newton para bases aditivas). $S e\{0,1\} \subseteq A, B \subseteq \mathbb{N} e$ $h \in \mathbb{Z}_{+}$, então

$$
h(A \cup B)=\bigcup_{i=0}^{h}[(h-i) A+i B] .
$$

Demonstração: Por indução sobre $h$. Se $h=1$, então

$$
\bigcup_{i=0}^{1}[(1-i) A+i B]=(A+0 B) \cup(0 A+B)=(A+\{0\}) \cup(\{0\}+B)=A \cup B .
$$

Suponha então que

$$
h(A \cup B)=\bigcup_{i=0}^{h}[(h-i) A+i B] .
$$

Utilizando os lemas desta seção, temos

$$
\begin{aligned}
(h+1)(A \cup B) & =h(A \cup B)+(A \cup B) \\
& =\left\{\bigcup_{i=0}^{h}[(h-i) A+i B]\right\}+A \cup B \\
& =\bigcup_{i=0}^{h}\{[(h-i) A+i B]+A \cup B\} \\
& =\bigcup_{i=0}^{h}[(h-i) A+i B+A] \cup[(h-i) A+i B+B] \\
& =\bigcup_{i=0}^{h}[(h-i+1) A+i B] \cup[(h-i) A+(i+1) B] \\
& =\bigcup_{i=0}^{h+1}[(h+1-i) A+i B] .
\end{aligned}
$$




\subsection{Bases aditivas finas em $(\mathcal{A}, d)$}

Pode-se pensar que, dada a "pouca" quantidade de elementos em uma base fina, elas sejam difíceis de encontrar em meio ao espaço métrico $(\mathcal{A}, d)$. Esta seção mostrará que isto não é verdade. Na verdade, o conjunto das bases finas é denso em $(\mathcal{A}, d)$. Denotaremos por $\mathcal{F}$ o conjunto das bases finas.

Vamos começar com os lemas preparatórios.

Lema 3.2.1. Sejam $A \in \mathcal{A}, h \in \mathbb{Z}_{+}$e $c>0$ tais que $A(n) \leq c \sqrt[h]{n}, \forall n \in \mathbb{Z}_{+}$. Então $o(A) \geq h$.

Demonstração: O resultado é trivial se $h=1$. Suponha $h \geq 2$ e suponha por absurdo que $o(A) \leq h-1$. Então $(h-1) \cdot A=\mathbb{N}$. Se $n \in \mathbb{Z}_{+}$, então

$$
n=((h-1) A)(n) \leq[1+A(n)]^{h-1} \leq[2 A(n)]^{h-1} \leq(2 c \sqrt[h]{n})^{h-1}=(2 c)^{h-1} n^{(h-1) / h} .
$$

Dividindo por $n$, obtemos

$$
1 \leq(2 c)^{h-1} n^{-1 / h} \rightarrow 0, \text { se } n \rightarrow \infty
$$

absurdo.

Lema 3.2.2. Sejam $A$ uma base fina de ordem $h$ e $B_{1}, \ldots, B_{m} \subseteq \mathbb{N}$ tais que

$$
B_{i}(n) \ll \sqrt[h]{n}, \forall i \in \overleftarrow{m}
$$

Então

$$
B=A \cup B_{1} \cup \cdots \cup B_{m}
$$

é uma base fina de ordem $h$.

Demonstração: Por hipótese, existem constantes $c, c_{1}, \ldots, c_{m}>0$ tais que

$$
A(n) \leq c \sqrt[h]{n}, \forall n \in \mathbb{Z}_{+} \quad \text { e } \quad B_{i}(n) \leq c_{i} \sqrt[h]{n}, \forall n \in \mathbb{Z}_{+}, \forall i \in \overleftarrow{m}
$$

Se $n \in \mathbb{Z}_{+}$, então

$B(n)=\left(A \cup B_{1} \cup \cdots \cup B_{m}\right)(n) \leq A(n)+B_{1}(n)+\cdots+B_{m}(n) \leq\left(c+c_{1}+\cdots+c_{m}\right) \sqrt[h]{n}$.

Pelo lema 3.2.1, $o(B) \geq h$. Mas como $B \supseteq A$, então $o(B) \leq o(A)=h$, o que implica $o(B)=h$.

Corolário 3.2.3. Se A for uma base fina de ordem $h e \Upsilon \subseteq \mathbb{N}$ for finito, então $A \cup \Upsilon$ será uma base fina de ordem $h$.

Corolário 3.2.4. Fixado $h \in \mathbb{Z}_{+}$, a união finita de bases finas de ordem $h$ é uma base fina de ordem $h$.

Se $\{0,1\} \subseteq A \subseteq \mathbb{N}$ e $m \in \mathbb{N}$, denotaremos $A+m=\{a+m ; a \in A\}$ para simplificar a notação. 
Lema 3.2.5. Se A for uma base fina de ordem $h$ e $m \in \mathbb{N}$, então

$$
B=\bigcup_{i=0}^{m}(A+i)
$$

é uma base fina de ordem $h$.

Demonstração: Como $A$ é uma base fina de ordem $h$, existe $c>0$ tal que

$$
A(n) \leq c \sqrt[h]{n}, \forall n \in \mathbb{Z}_{+} .
$$

Seja $n \in \mathbb{Z}_{+}$. Então

$B(n)=\left(\bigcup_{i=0}^{m}(A+i)\right)(n) \leq \sum_{i=0}^{m}(A+i)(n) \leq \sum_{i=0}^{m} A(n)=(m+1) \cdot A(n) \leq(m+1) c \sqrt[h]{n}$.

Pelo lema 3.2.1, $o(B) \geq h$. Mas $B \supseteq A$, então $o(B) \leq o(A)=h$. Logo $o(B)=h$.

Lema 3.2.6. Sejam $A$ e $B$ bases aditivas de ordem $h$. Se A for fina $e$

$$
(B-A)(n) \ll \sqrt[h]{n},
$$

então $B$ será fina.

Demonstração: Por hipótese, existem constantes $c, c^{\prime}>0$ tais que

$$
A(n) \leq c \sqrt[h]{n}, \forall n \in \mathbb{Z}_{+} \quad \text { e } \quad(B-A)(n) \leq c^{\prime} \sqrt[h]{n}, \forall n \in \mathbb{Z}_{+} .
$$

Logo,

$$
B(n)=(A \cap B)(n)+(B-A)(n) \leq c \sqrt[h]{n}+c^{\prime} \sqrt[h]{n}=\left(c+c^{\prime}\right) \sqrt[h]{n}, \forall n \in \mathbb{Z}_{+},
$$

o que implica que $B$ é fina.

Com estes lemas conseguimos provar que $\overline{\mathcal{F}}=\mathcal{A}$. Vale destacar o caráter artesanal da demonstração abaixo.

Teorema 3.2.7. O conjunto das bases finas é denso em $(\mathcal{A}, d)$.

Demonstração: Como

$$
E=\{A \in \mathcal{A} ; \mathbb{N}-A \text { é finito }\}
$$

é denso em $(\mathcal{A}, d)$ (proposição 2.2.5), é suficiente mostrar que $\overline{\mathcal{F}} \supseteq E$. Para isto, sejam $A \in E$ e $\varepsilon>0$ dados, e suponha que $A \neq \mathbb{N}$ para evitar o caso trivial. Então

$$
o(A)=h \geq 2
$$

$\mathrm{e}$

$$
A=\{0,1\} \cup \widetilde{A} \cup\left\{n_{0}, n_{0}+1, \ldots\right\},
$$

onde $n_{0}$ é o menor número natural tal que se $n \geq n_{0}$, então $n \in A$. Isto implica que $n_{0} \geq 3$ e que

$$
\widetilde{A} \subseteq\left\{2, \ldots, n_{0}-1\right\}
$$


Seja $F_{h}$ a base fina de Raikov-Stöhr de ordem $h$ e seja $n_{1} \in \mathbb{Z}_{+}$tal que

$$
\sum_{\alpha>n_{1}} \frac{1}{\alpha^{2}}<\varepsilon,
$$

e suponha sem perda de generalidade que $n_{1} \geq h n_{0}$.

Seja

$$
T=\bigcup_{i=0}^{(h-1) n_{0}} F_{h}+i,
$$

que, pelo lema 3.2.5, é uma base fina de ordem $h$. Seja

$$
B=\{0,1\} \cup \widetilde{A} \cup\left\{n_{0}, \ldots, n_{1}\right\} \cup\left(T \cap\left\{x \in \mathbb{N} ; x>n_{1}\right\}\right) .
$$

Afirmo que

1. $B \in \mathcal{A}$ e $o(B)=o(A)$;

2. $d(A, B)<\varepsilon ; \mathrm{e}$,

3. $B$ é fina.

Isto é suficiente para provar o teorema.

1. Como

$$
B \cap\left\{x \in \mathbb{N} ; x \leq n_{1}\right\}=A \cap\left\{x \in \mathbb{N} ; x \leq n_{1}\right\},
$$

se $n \in \mathbb{N}$ e $n \leq n_{1}$, então

$$
o(n, B)=o(n, A) \leq o(A) .
$$

Além disso, $\operatorname{como} \operatorname{ppc}(A)<n_{0}$, então

$$
o(A)=o(\operatorname{ppc}(A), A)=o(\operatorname{ppc}(A), B) \leq o(B) .
$$

Assim, para provar este item é suficiente mostrar que

$$
o(n, B) \leq o(A), \forall n \in \mathbb{N}, n>n_{1} .
$$

Para isto, seja $n>n_{1}$. Como $o\left(F_{h}\right)=h$, existem $t_{1}, \ldots, t_{h} \in F_{h}$ tais que

$$
n=t_{1}+\cdots+t_{h}
$$

Note que nem todos os $t_{i}$ são menores do que $n_{0}$, porque se este fosse o caso, então

$$
n<h n_{0} \leq n_{1}
$$

absurdo. Então existe $i \in \overleftarrow{h}$ tal que $t_{i} \geq n_{0}$. Se $t_{1}, \ldots, t_{h} \geq n_{0}$, então $t_{1}, \ldots, t_{h} \in B$ o que implica que $o(n, B) \leq h$. Caso contrário, existe $i^{\prime} \in \overleftarrow{h}$ tal que $t_{i^{\prime}}<n_{0}$. Seja $j \in \overleftarrow{h-1}$ tal que

$$
\left\{\begin{array}{c}
t_{1}, \ldots, t_{j} \geq n_{0} \\
t_{j+1}, \ldots, t_{h}<n_{0}
\end{array}\right.
$$


Note que

$$
t_{j+1}+\cdots+t_{h}<(h-j) n_{0} \leq(h-1) n_{0} .
$$

Como $t_{j} \in F_{h} \mathrm{e}$

$$
0 \leq t_{j+1}+\cdots+t_{h}<(h-1) n_{0},
$$

então

$$
t_{j}+t_{j+1}+\cdots+t_{h} \in T
$$

Como

$$
t_{j}+t_{j+1}+\cdots+t_{h} \geq t_{j} \geq n_{0},
$$

então

$$
t_{j}+t_{j+1}+\cdots+t_{h} \in B
$$

Assim podemos escrever

$$
n=\underbrace{t_{1}}_{\in B}+\cdots+\underbrace{t_{j-1}}_{\in B}+\underbrace{t_{j}+t_{j+1}+\cdots+t_{h}}_{\in B},
$$

o que implica

$$
o_{B}(n) \leq j \leq h-1<o(A)
$$

Logo $o(B)=o(A)$

$$
\text { 2. } d(A, B)=\underbrace{|o(A)-o(B)|}_{=0}+\sum_{\alpha \in A \triangle B} \frac{1}{\alpha^{2}}=\sum_{\alpha \in A \triangle B} \frac{1}{\alpha^{2}} \leq \sum_{\alpha>n_{1}} \frac{1}{\alpha^{2}}<\varepsilon \text {. }
$$

3. Como $B-T$ é um conjunto finito, o lema 3.2.6 implica que $B$ é fina de ordem $h$.

O fato de $\mathcal{F}$ ser denso em $\mathcal{A}$ será usado na próxima seção para mostrar que algumas funções $f: \mathcal{A} \rightarrow \mathcal{A}$ têm propriedades muito boas com relação à função ordem.

\subsection{Funções de $\mathcal{A}$ em $\mathcal{A}$}

Esta seção estudará três tipos de funções de $\mathcal{A}$ em $\mathcal{A}$. Ao final da seção, daremos um critério de quando podemos colocar uma quantidade infinita de números em uma base aditiva sem alterar sua ordem. O primeiro tipo de função de $\mathcal{A}$ em $\mathcal{A}$ que estudaremos são as funções pseudocontínuas.

Definição 3.3.1. Dizemos que $f: \mathcal{A} \rightarrow \mathcal{A}$ é pseudocontínua em $A \in \mathcal{A}$ se $A_{n} \rightarrow A$ implicar $f\left(A_{n}\right) \stackrel{p}{\rightarrow} f(A)$. Dizemos que $f: \mathcal{A} \rightarrow \mathcal{A}$ é pseudocontínua em $X \subseteq \mathcal{A}$ se $f$ for pseudocontínua em $A, \forall A \in X$.

Vejamos dois exemplos básicos de funções pseudocontínuas.

Exemplo 3.3.2. $S e \Upsilon \subseteq \mathbb{N} e$

$$
\begin{aligned}
f_{\Upsilon}: \mathcal{A} & \rightarrow \mathcal{A} \\
A & \mapsto A \cup \Upsilon
\end{aligned}
$$

então $f_{\Upsilon}$ é uma função pseudocontínua em $\mathcal{A}$. 
Exemplo 3.3.3. Se $\{1\} \subseteq \Upsilon \subseteq \mathbb{Z}_{+} e$

$$
\begin{aligned}
g_{\Upsilon}: \mathcal{A} & \rightarrow \mathcal{A} \\
A & \mapsto \bigcup_{i \in \Upsilon} A^{i}
\end{aligned}
$$

então $g_{\Upsilon}$ é uma função pseudocontínua em $\mathcal{A}$.

Outro tipo de função de $\mathcal{A}$ em $\mathcal{A}$ que estudaremos são as funções ordenativas.

Definição 3.3.4. Dizemos que $f: \mathcal{A} \rightarrow \mathcal{A}$ é ordenativa em $A \in \mathcal{A}$ se existir $r>0$ tal que se $d(A, B)<r$, então $o(f(A))=o(f(B))$. Dizemos que uma função $f: \mathcal{A} \rightarrow \mathcal{A}$ é ordenativa em $X \subseteq \mathcal{A}$ se $f$ for ordenativa em $A, \forall A \in X$.

Basicamente, as propriedades de pseudocontinuidade e ordenatividade de funções $f: \mathcal{A} \rightarrow \mathcal{A}$ codificam a continuidade de $f$ em uma determinada base $A \in \mathcal{A}$, conforme o teorema abaixo.

Teorema 3.3.5. Seja $A \in \mathcal{A}$. As seguintes afirmações são equivalentes com respeito a uma função $f: \mathcal{A} \rightarrow \mathcal{A}$.

1. $f$ é contínua em A;

2. $f$ é ordenativa e pseudocontínua em A.

Demonstração: (1. $\Rightarrow$ 2.) Suponha que $f$ é contínua em $A$. Então dado

$$
\varepsilon=\frac{1}{2}
$$

existe $\delta>0$ tal que se $d(A, B)<\delta$, então

$$
d(f(A), f(B))<\frac{1}{2} .
$$

Em particular, se $d(A, B)<\delta$, então

$$
o(f(A))=o(f(B))
$$

ou seja, $f$ é ordenativa em $A$. Para mostrar que $f$ é pseudocontínua em $A$, seja $\left\{A_{n}\right\}_{n \in \mathbb{N}}$ uma sequência em $\mathcal{A}$ tal que $A_{n} \rightarrow A$. Como $f$ é contínua em $A$,

$$
f\left(A_{n}\right) \rightarrow f(A) .
$$

Pela proposição 2.2.9,

$$
f\left(A_{n}\right) \stackrel{p}{\rightarrow} f(A) .
$$

(2. $\Rightarrow$ 1.) Seja $\left\{A_{n}\right\}_{n \in \mathbb{N}}$ uma sequência em $\mathcal{A}$ tal que $A_{n} \rightarrow A$. Vamos mostrar que $f\left(A_{n}\right) \rightarrow f(A)$. Para isto, seja $\varepsilon>0$. Como $f$ é pseudocontínua em $A$,

$$
f\left(A_{n}\right) \stackrel{p}{\rightarrow} f(A) .
$$


Logo existe $n_{0} \in \mathbb{N}$ tal que se $n \geq n_{0}$, então

$$
\sum_{\alpha \in f\left(A_{n}\right) \triangle f(A)} \frac{1}{\alpha^{2}}<\varepsilon .
$$

Agora, como $f$ é ordenativa em $A$, existe $r>0$ tal que se $d(A, B)<r$, então

$$
o(f(A))=o(f(B)) .
$$

Como $A_{n} \rightarrow A$, existe $n_{1} \in \mathbb{N}$ tal que se $n \geq n_{1}$, então $d\left(A_{n}, A\right)<r$, o que implica

$$
o\left(f\left(A_{n}\right)\right)=o(f(A)), \forall n \geq n_{1} .
$$

Se $n_{2}=\max \left\{n_{0}, n_{1}\right\}$ e se $n \geq n_{2}$, então

$$
d\left(f\left(A_{n}\right), f(A)\right)=\left|o\left(f\left(A_{n}\right)\right)-o(f(A))\right|+\sum_{\alpha \in f\left(A_{n}\right) \triangle f(A)} \frac{1}{\alpha^{2}}=\sum_{\alpha \in f\left(A_{n}\right) \triangle f(A)} \frac{1}{\alpha^{2}}<\varepsilon .
$$

Logo $f\left(A_{n}\right) \rightarrow f(A)$.

Os exemplos abaixo mostram que estes conceitos são também distintos entre si.

Exemplo 3.3.6. A função

$$
\begin{aligned}
& f_{1}: \mathcal{A} \rightarrow \quad \mathcal{A} \\
& A \mapsto\left\{\begin{array}{cl}
A & \text {, se } A \neq \mathbb{N}-\{2\} \\
\mathbb{N}-\{3\} & \text {, se } A=\mathbb{N}-\{2\}
\end{array}\right.
\end{aligned}
$$

é ordenativa em $\mathcal{A}$, mas não é contínua em $\mathbb{N}-\{2\}$.

De fato,

$$
\mathbb{N}-\{2, n\} \rightarrow \mathbb{N}-\{2\}, \text { se } n \rightarrow \infty,
$$

mas

$$
f_{1}(\mathbb{N}-\{2, n\}) \not \rightarrow \mathbb{N}-\{3\}, \text { se } n \rightarrow \infty .
$$

Exemplo 3.3.7. A função

$$
\begin{aligned}
f_{2}: \mathcal{A} & \rightarrow \mathcal{A} \\
A & \mapsto A \cup\{2\}
\end{aligned}
$$

é pseudocontínua em $\mathcal{A}$ mas não é contínua em $\mathbb{N}-\{2\}$.

Com efeito,

$$
\mathbb{N}-\{2, n\} \rightarrow \mathbb{N}-\{2\}, \text { se } n \rightarrow \infty,
$$

mas

$$
f_{2}(\mathbb{N}-\{2, n\}) \not \rightarrow \mathbb{N}, \text { se } n \rightarrow \infty .
$$

Se $f: \mathcal{A} \rightarrow \mathcal{A}$ for uma função, denotaremos

$$
\operatorname{cont}(f)=\{A \in \mathcal{A} ; f \text { é contínua em } \mathcal{A}\}
$$

para simplificar a notação. 
Proposição 3.3.8. Se $f: \mathcal{A} \rightarrow \mathcal{A}$ for uma função pseudocontínua em $\mathcal{A}$, então $\operatorname{cont}(f)$ é um subconjunto aberto de $\mathcal{A}$. Além disso, se $K$ for um subconjunto compacto de $\mathcal{A}$, então $f(K)$ é um subconjunto fechado de $\mathcal{A}$.

Demonstração: Seja $A \in \operatorname{cont}(f)$. Dado

$$
\varepsilon=\frac{1}{2}
$$

existe $\delta>0$ tal que se $d(A, B)<\delta$, então

$$
d(f(A), f(B))<\frac{1}{2} .
$$

Em particular,

$$
o(f(A))=o(f(B)), \forall B \in \mathcal{B}(A, \delta) .
$$

Afirmo que

$$
\mathcal{B}(A, \delta) \subseteq \operatorname{cont}(f)
$$

De fato, seja

$$
B \in \mathcal{B}(A, \delta) .
$$

Como $f$ é pseudocontínua em $B$, pelo teorema 3.3.5 é suficiente mostrar que $f$ é ordenativa em $B$. Para isto, seja $r>0$ tal que

$$
\mathcal{B}(B, r) \subseteq \mathcal{B}(A, \delta) .
$$

Se

$$
C \in \mathcal{B}(B, r)
$$

então

$$
C \in \mathcal{B}(A, \delta)
$$

o que implica

$$
o(f(C))=o(f(A))=o(f(B)) .
$$

Como $C$ é qualquer, $f$ é ordenativa em $B$ e, portanto, contínua em $B$.

Agora suponha que $K$ seja um subconjunto compacto de $\mathcal{A}$ e seja $\left\{f\left(A_{n}\right)\right\}_{n \in \mathbb{N}}$ uma sequência em $f(K)$ tal que

$$
f\left(A_{n}\right) \rightarrow B \in \mathcal{A}
$$

Precisamos mostrar que $B \in f(K)$. Como $K$ é compacto, a sequência $\left\{A_{n}\right\}_{n \in \mathbb{N}}$ admite uma subsequência convergente para, digamos, $B^{\prime} \in K$. Suponha sem perda de generalidade que a própria sequência converge para $B^{\prime}$. Como $A_{n} \rightarrow B^{\prime}$, e $f$ é pseudocontínua, então

$$
f\left(A_{n}\right) \stackrel{p}{\rightarrow} f\left(B^{\prime}\right) .
$$

Como $f\left(A_{n}\right) \rightarrow B$, então

$$
f\left(A_{n}\right) \stackrel{p}{\rightarrow} B .
$$

Pela unicidade do limite, $B=f\left(B^{\prime}\right)$, o que implica que $B \in f(K)$.

O terceiro tipo de função que estudaremos são as funções expansivas. 
Definição 3.3.9. Dizemos que $f: \mathcal{A} \rightarrow \mathcal{A}$ é expansiva se $f(A) \supseteq A, \forall A \in \mathcal{A}$.

Proposição 3.3.10. Seja $f: \mathcal{A} \rightarrow \mathcal{A}$ uma função expansiva. Se $f$ for pseudocontínua em $A \in \mathcal{A}$ e se $o(A)=o(f(A))$, então $f$ é contínua em $A$.

Demonstração: Sejam $\left\{A_{n}\right\}_{n \in \mathbb{N}}$ uma sequência em $\mathcal{A}$ tal que $A_{n} \rightarrow A$ e $\varepsilon>0$ dados, e suponha que $A \neq \mathbb{N}$ para evitar o caso trivial. Como $A_{n} \rightarrow A$, existe $n_{0} \in \mathbb{N}$ tal que se $n \geq n_{0}$, então

$$
d\left(A, A_{n}\right)<\frac{1}{2}
$$

o que implica

$$
o(A)=o\left(A_{n}\right), \forall n \geq n_{0}
$$

Como

$$
A_{n} \subseteq f\left(A_{n}\right), \forall n \in \mathbb{N}
$$

então

$$
o\left(f\left(A_{n}\right)\right) \leq o\left(A_{n}\right)=o(A)=o(f(A)), \forall n \geq n_{0} .
$$

Agora, como $f$ é pseudocontínua em $A$, então

$$
f\left(A_{n}\right) \stackrel{p}{\rightarrow} f(A) .
$$

Note que como $A \neq \mathbb{N}$ e $o(A)=o(f(A))$, então $f(A) \neq \mathbb{N}$, o que implica $\operatorname{ppc}(f(A)) \geq$ 2. Assim existe $n_{1} \in \mathbb{N}$ tal que

$$
\sum_{\alpha \in f\left(A_{n}\right) \triangle f(A)} \frac{1}{\alpha^{2}}<\frac{1}{\operatorname{ppc}(f(A))^{2}}, \forall n \geq n_{1} .
$$

Em particular,

$$
f\left(A_{n}\right) \cap \overleftarrow{\operatorname{ppc}(f(A))}=f(A) \cap \overleftarrow{\operatorname{ppc}(f(A))}, \forall n \geq n_{1}
$$

Portanto

$$
o\left(f\left(A_{n}\right)\right) \geq o\left(\operatorname{ppc}(f(A)), f\left(A_{n}\right)\right)=o(\operatorname{ppc}(f(A)), f(A))=o(f(A)), \forall n \geq n_{1} .
$$

Além disso, existe $n_{2} \in \mathbb{N}$ tal que se $n \geq n_{2}$, então

$$
\sum_{\alpha \in f\left(A_{n}\right) \triangle f(A)} \frac{1}{\alpha^{2}}<\varepsilon .
$$

Portanto, se

$$
n_{3}=\max \left\{n_{0}, n_{1}, n_{2}\right\}
$$

e se $n \geq n_{3}$, então

$$
d\left(f\left(A_{n}\right), f(A)\right)=\left|o\left(f\left(A_{n}\right)\right)-o(f(A))\right|+\sum_{\alpha \in f\left(A_{n}\right) \triangle f(A)} \frac{1}{\alpha^{2}}=\sum_{\alpha \in f\left(A_{n}\right) \triangle f(A)} \frac{1}{\alpha^{2}}<\varepsilon,
$$

o que implica

$$
f\left(A_{n}\right) \rightarrow f(A),
$$

mostrando assim que $f$ é contínua em $A$. 
Lema 3.3.11. Seja $f: \mathcal{A} \rightarrow \mathcal{A}$ e suponha que $f$ é contínua em $A \in \mathcal{A}$. Suponha que dado $\delta>0$ exista $A_{\delta} \in \mathcal{A}$ tal que $d\left(A, A_{\delta}\right)<\delta$ e o $\left(A_{\delta}\right)=o\left(f\left(A_{\delta}\right)\right)$. Então $o(A)=o(f(A))$.

Demonstração: Como $f$ é contínua em $A$, dado

$$
\varepsilon=\frac{1}{2}
$$

existe $\delta>0$ tal que se $d(A, B)<\delta$, então

$$
d(f(A), f(B))<\varepsilon .
$$

Em particular, isto implica

$$
o(f(A))=o(f(B)), \forall B \in \mathcal{B}(A, \delta) .
$$

Por hipótese, existe $A_{\delta} \in \mathcal{A}$ tal que $d\left(A, A_{\delta}\right)<\delta$ e $o\left(A_{\delta}\right)=o\left(f\left(A_{\delta}\right)\right)$. Suponha sem perda de generalidade que $\delta<1$. Então

$$
o(A)=o\left(A_{\delta}\right)=o\left(f\left(A_{\delta}\right)\right)=o(f(A)) .
$$

Vamos agora aplicar os resultados desta seção para uma função em particular, a chamada função potencializadora, definida pela regra

$$
\begin{aligned}
\mathcal{G}: \mathcal{A} & \rightarrow \mathcal{A} \\
A & \mapsto \bigcup_{i=1}^{\infty} A^{i}
\end{aligned}
$$

Para isto, precisamos antes de um lema preparatório.

Lema 3.3.12. Se $A \in \mathcal{A}$, então $\mathcal{G}(A)(n) \leq \frac{\ln (n) A(n)}{\ln (2)}, \forall n \geq 2$.

Demonstração: Sejam $n \geq 2$ e

$$
k=\left\lfloor\frac{\ln (n)}{\ln (2)}\right\rfloor .
$$

Como

$$
\mathcal{G}(A) \cap \overleftarrow{n} \subseteq\left(\bigcup_{i=1}^{k} A^{i}\right) \cap \overleftarrow{n}
$$

então

$$
\mathcal{G}(A)(n) \leq\left(\bigcup_{i=1}^{k} A^{i}\right)(n) \leq \sum_{i=1}^{k} A^{i}(n) \leq \sum_{i=1}^{k} A(n) \leq \frac{\ln (n) A(n)}{\ln (2)}
$$

São nos dois resultados abaixo que podemos ver uma grande importância do fato de $\mathcal{F}$ ser denso em $\mathcal{A}$. 
Proposição 3.3.13. Se $A \in \mathcal{A}$ for uma base fina de ordem $h$, então $o(A)=o(\mathcal{G}(A))$.

Demonstração: Por hipótese, existe $c>0$ tal que

$$
A(n) \leq c n^{1 / h}, \forall n \in \mathbb{Z}_{+} .
$$

Suponha por absurdo que $o(A) \neq o(\mathcal{G}(A))$. Como $\mathcal{G}$ é uma função expansiva, $o(\mathcal{G}(A))<o(A)$, o que implica $o(A) \geq 2$. Seja $k=h-1 \in \mathbb{Z}_{+}$. Portanto

$$
k \cdot \mathcal{G}(A)=\mathbb{N}
$$

ou seja,

$$
[k \cdot \mathcal{G}(A)](n)=n, \forall n \in \mathbb{Z}_{+} .
$$

Dessa forma, se $n \geq 2$, segue do lema 3.3.12 que

$$
n \leq[1+\mathcal{G}(A)(n)]^{k} \leq 2^{k} \mathcal{G}(A)(n)^{k} \leq 2^{k}\left[\frac{\ln (n) A(n)}{\ln (2)}\right]^{k} \leq\left(\frac{2 c}{\ln (2)}\right)^{k} \ln (n)^{k} n^{k /(k+1)}
$$

Dividindo por $n$,

$$
1 \leq\left(\frac{2 c}{\ln (2)}\right)^{k} \ln (n)^{k} n^{-1 /(k+1)} \rightarrow 0, \text { se } n \rightarrow \infty,
$$

absurdo. $\operatorname{Logo} o(\mathcal{G}(A))=o(A)$.

Teorema 3.3.14. Seja $A \in \mathcal{A}$. As seguintes afirmações são equivalentes:

- $\mathcal{G}$ é contínua em $A$;

- $o(A)=o(\mathcal{G}(A))$.

Demonstração: (1. $\Rightarrow$ 2.) Suponha $\mathcal{G}$ contínua em $A$. Pela proposição 3.3.13,

$$
o(B)=o(\mathcal{G}(B)), \forall B \in \mathcal{F} .
$$

Como $\mathcal{F}$ é denso em $\mathcal{A}$, então $o(A)=o(\mathcal{G}(A))$ pelo lema 3.3.11.

(2. $\Rightarrow$ 1.) Basta observar que $\mathcal{G}$ é pseudocontínua e aplicar a proposição 3.3.10.

Na verdade, se "olharmos feio" para a demonstração do teorema 3.3.14, qualquer função que se comporte de maneira análoga à função $\mathcal{G}$ com relação a algum conjunto de números e a algum subconjunto denso de $\mathcal{A}$ também satisfará a tese do teorema 3.3.14.

Teorema 3.3.15. Seja $f: \mathcal{A} \rightarrow \mathcal{A}$ uma função e fixe $A \in \mathcal{A}$. Se f for expansiva em $\mathcal{A}$, pseudocontínua em $\mathcal{A}$ e se existir $D \subseteq \mathcal{A}$ denso em $\mathcal{A}$ tal que

$$
o(B)=o(f(B)), \forall B \in D
$$

então as seguintes afirmações são equivalentes: 
- $f$ é contínua em A;

- $o(A)=o(f(A))$.

Demonstração: A demonstração é inteiramente análoga à do teorema 3.3.14.

A importância do teorema 3.3.15 reside no fato de que podemos utilizar informações assintóticas sobre certos conjuntos numéricos para obter informações gerais sobre os mesmos. Por exemplo, o teorema de Vinogradov (teorema 1.1.10) afirma que todo número ímpar suficientemente grande pode ser escrito como a soma de três números primos. Em particular, isto implica

$$
o_{\infty}\left(\mathbb{P}^{*}\right) \leq 4
$$

Agora considere a função

$$
\begin{aligned}
f_{\Upsilon}: \mathcal{A} & \rightarrow \mathcal{A} \\
A & \mapsto A \cup \Upsilon
\end{aligned}
$$

do exemplo 3.3.2, onde $\Upsilon \subseteq \mathbb{N}$ é um conjunto finito qualquer. Esta função é expansiva, pseudocontínua, e se $D=\mathcal{F}$, segue do corolário 3.2 .3 que

$$
o(B)=o\left(f_{\Upsilon}(B)\right), \forall B \in D
$$

Assim, se conseguirmos mostrar que $f_{\Upsilon}$ é contínua em $\mathbb{P}^{*}$, qualquer que seja $\Upsilon$ finito, seguirá do teorema 3.3 .15 que

$$
o\left(\mathbb{P}^{*}\right) \leq 4
$$

O mesmo raciocínio vale para a função $\mathcal{G}$. Se mostrarmos que $\mathcal{G}$ é contínua em $\mathbb{P}^{*}$, então o teorema 3.3.15 implicará

$$
o\left(\mathbb{P}^{*}\right)=o\left(\mathcal{G}\left(\mathbb{P}^{*}\right)\right)=o\left(\bigcup_{i=1}^{\infty} \mathbb{P}^{i}\right)
$$

ou seja, teremos "mais números" para utilizar no estudo da conjectura de Goldbach.

Vale notar que a ideia da demonstração do teorema 3.3.15 nos mostra que, sob certas condições, nós podemos acrescentar uma certa quantidade de números a uma determinada base aditiva sem alterar sua ordem. 


\section{Capítulo 4}

\section{O problema das P.A.'s em bases aditivas}

Fixada uma base $A \in \mathcal{A}$, definimos o conjunto excepcional de $A$ por

$$
\operatorname{ex}(A)=\{n \in \mathbb{N} ; o(n, A)=o(A)\}=\mathbb{N}-(o(A)-1) A .
$$

Dessa forma, inspirados no teorema 1.1.4, uma pergunta interessante que podemos fazer a cada base aditiva $A$ é a seguinte: existe alguma maneira de caracterizar ex $(A)$ ? De maneira mais fraca, o que pode-se falar a respeito de $\mathbb{N}-t \cdot A$, $t \in \overleftarrow{o(A)}$ ?

Este capítulo versa sobre este problema, centrando esforços na busca por progressões aritméticas em conjuntos da forma $\mathbb{N}-t \cdot A, t \in \overleftarrow{o(A)}$. Enquanto que a primeira seção apresenta um exemplo básico sobre este tópico, a seção subsequente apresenta uma aplicação inesperada de um teorema de J. Singer sobre este assunto.

\subsection{O problema das P.A.'s}

Como dito na introdução, fixada uma base aditiva $A \in \mathcal{A}$, o que pode ser dito a respeito de $\operatorname{ex}(A)$ ? Bem, sabemos que

$$
\operatorname{ex}(A) \neq \emptyset
$$

e que

$$
\min (\operatorname{ex}(A))=\operatorname{ppc}(A)
$$

Mas quais outras informações podemos obter sobre ex $(A)$ ?

É claro que ex $(A)$ pode ter um único elemento, como é o caso de $A=\mathbb{N}-\{2\}$, ou ter uma infinidade de elementos, como é o caso de $A=\mathbb{N}-\left(\mathbb{N}^{2}-\{0,1\}\right)$. Note ainda que no segundo exemplo dado, $\operatorname{ex}(A)$ é um conjunto infinito que não admite nenhuma progressão aritmética não trivial. 
Este fenômeno não ocorre com $\mathbb{N}^{2}$. Pelo teorema 1.1.4,

$$
\{8 b+7 ; b \in \mathbb{N}\} \subseteq \operatorname{ex}\left(\mathbb{N}^{2}\right)
$$

Vejamos outro exemplo de base aditiva que contém uma progressão aritmética em seu conjunto excepcional.

Proposição 4.1.1. Se $a \in \mathbb{N}$ e $a \equiv 5$ ou $8(\bmod 9)$, então $a \in \operatorname{ex}\left(P_{3}\right)$.

Demonstração: Suponha $a \equiv 5(\bmod 9)$. Então existe $b \in \mathbb{N}$ tal que

$$
a=5+9 b .
$$

Suponha por absurdo que a possa ser escrito como a soma de dois números triangulares. Então existem $n, m \in \mathbb{N}$ tais que

$$
5+9 b=\frac{n(n+1)}{2}+\frac{m(m+1)}{2},
$$

ou seja,

$$
10+18 b=n(n+1)+m(m+1) .
$$

Tomando esta equação módulo 3, temos

$$
1 \equiv n(n+1)+m(m+1) \quad(\bmod 3)
$$

Isto implica

$$
n \equiv 1 \quad(\bmod 3) \quad \text { e } \quad m \equiv 1 \quad(\bmod 3) .
$$

Portanto existem $n^{\prime}, m^{\prime} \in \mathbb{N}$ tais que

$$
n=3 n^{\prime}+1 \quad \text { e } \quad m=3 m^{\prime}+1 .
$$

Dessa forma,

$10+18 b=\left(3 n^{\prime}+1\right)\left(3 n^{\prime}+2\right)+\left(3 m^{\prime}+1\right)\left(3 m^{\prime}+2\right)=9 n^{\prime 2}+9 n^{\prime}+2+9 m^{\prime 2}+9 m^{\prime}+2$.

Tomando esta equação módulo 9, obtemos

$$
1 \equiv 4 \quad(\bmod 9)
$$

absurdo. O caso $a \equiv 8(\bmod 9)$ é análogo.

Este tipo de argumento se generaliza para outras bases bem comportadas. Em particular, vamos agora utilizar a ideia da proposição acima para apresentar uma condição suficiente para a existência de uma progressão aritmética em $\mathbb{N}-(B+B)$, onde $B$ é uma base aditiva satisfazendo certas condições. 


\subsection{Uma aplicação do teorema de Singer}

Para iniciar esta seção vamos apresentar o teorema de Singer.

Teorema 4.2.1 (Singer, 1938). Seja $p \in \mathbb{P}$ e defina $q=1+p+p^{2}$. Então existe um conjunto

$$
A \subseteq \mathbb{Z} / q \mathbb{Z}
$$

tal que

$$
\#(A)=p+1
$$

$e$

$$
A+A=\mathbb{Z} / q \mathbb{Z}-\{0\} .
$$

Recomendamos [80] para uma prova deste teorema. Abaixo, vamos apresentar uma maneira de construir bases aditivas $B \in \mathcal{A}$ tais que $\mathbb{N}-(B+B)$ contém uma progressão aritmética. Para fazer isto, precisamos do seguinte lema.

Lema 4.2.2. Seja $q \in \mathbb{P}$. Então toda função $f: \mathbb{Z} / q \mathbb{Z} \rightarrow \mathbb{Z} / q \mathbb{Z}$ é representada unicamente por um polinômio de grau no máximo $q-1$.

Demonstração: Existem $q^{q}$ funções de $\mathbb{Z} / q \mathbb{Z}$ em $\mathbb{Z} / q \mathbb{Z}$, assim como existem $q^{q}$ polinômios de graus no máximo $q-1 \mathrm{em} \mathbb{Z} / q \mathbb{Z}$. Assim, basta mostrarmos que se $f(x), g(x) \in \mathbb{Z} / q \mathbb{Z}[x]$ representam a mesma função de $\mathbb{Z} / q \mathbb{Z}$ em $\mathbb{Z} / q \mathbb{Z}$ e ambos têm grau menor que $q$, então $f=g$. Para isto, note que se

$$
f(a)=g(a), \forall a \in \mathbb{Z} / q \mathbb{Z},
$$

então $h=f-g$ é um polinômio múltiplo de

$$
\prod_{i=0}^{q-1}(x-i)
$$

em $\mathbb{Z} / q \mathbb{Z}$. Como $q$ é primo, então $\mathbb{Z} / q \mathbb{Z}$ é um corpo, o que implica que ou $h=0$ ou o grau de $h$ é pelo menos $q$. Como os graus de $f$ e $g$ são menores que $q$, então $h=0$, ou seja, $f=g$.

Agora vamos construir a base aditiva $B$. Para isto, seja $p \in \mathbb{P}$ tal que $q=1+p+p^{2} \in \mathbb{P}$. Pelo teorema de Singer, existe

$$
A \subseteq \mathbb{Z} / q \mathbb{Z}
$$

tal que

$$
\#(A)=p+1
$$

$\mathrm{e}$

$$
A+A=\mathbb{Z} / q \mathbb{Z}-\{0\} .
$$

Seja

$$
f: \mathbb{Z} / q \mathbb{Z} \rightarrow \mathbb{Z} / q \mathbb{Z}
$$

não nula tal que

$$
\operatorname{Im}(f) \subseteq A
$$


Pelo lema anterior, $f$ pode ser representada por um polinômio em $\mathbb{Z} / q \mathbb{Z}[x]$. Levante $f$ para um polinômio $F[x] \in \mathbb{Z}[x]$ e suponha sem perda de generalidade que o coeficiente líder de $F[x]$ é positivo. Pelo teorema de Kamke (1.2.8), existe $n_{0} \in \mathbb{N}$ tal que

$$
B=\{0,1\} \cup\left\{F(n) ; n \geq n_{0}\right\}
$$

é uma base aditiva. Ademais, como a projeção de $B+B$ em $\mathbb{Z} / q \mathbb{Z}$ está contida em $A$, então $\mathbb{N}-(B+B)$ contém uma progressão aritmética. 


\section{Capítulo 5}

\section{Prime gaps e a conjectura de Firoozbakht}

Os números primos sempre exerceram fascínio sobre os matemáticos. Muito do desenvolvimento da teoria dos números, e até mesmo da própria Matemática, deve-se à curiosidade em tentar entender suas propriedades. Este capítulo versa sobre um problema muito antigo, e mesmo assim com intensa atividade de pesquisa nos dias de hoje, sobre números primos: o problema dos prime gaps. Se $n \in \mathbb{Z}_{+}$, o $n$-ésimo prime gap é definido pela equação

$$
g_{n}=p_{n+1}-p_{n},
$$

onde $p_{n}$ denota o $n$-ésimo número primo. Este capítulo inicia-se com um breve resumo do estado-da-arte na teoria dos prime gaps, para depois apresentar o enunciado da conjectura de Firoozbakht e algumas de suas consequências. Na seção final, é demonstrada uma relação bastante interessante entre o recente teorema de Zhang sobre a função $g_{n}$ e a conjectura de Firoozbakht.

\subsection{O estado-da-arte em prime gaps}

Iniciamos esta seção com o seguinte resultado clássico.

Teorema 5.1.1 (Euclides, $\pm 300 \mathrm{AC}$ ). Os números primos são infinitos.

Existe hoje uma quantidade muito variada de provas deste teorema, algumas das quais são verdadeiras pérolas do raciocínio lógico. Para uma coletânea de tais demonstrações, recomendamos [67]. Assim, dado um número primo $p_{n}$, sabemos de início apenas que existe $g_{n}$. Mas como se comporta a função $g_{n}$ ? É fácil ver que

$$
\limsup g_{n}=\infty \text {. }
$$

De fato, dado $n \in \mathbb{Z}_{+}$, a sequência

$$
(n+1) !+2,(n+1) !+3, \ldots,(n+1) !+(n+1)
$$

contém $n$ inteiros positivos compostos. Mas o primeiro resultado substancial sobre $g_{n}$ foi dado por Chebyshev. 
Teorema 5.1.2 (Bertrand-Chebyshev, 1852). Se $n \in \mathbb{N}$ e $n \geq 2$, então existe $p \in \mathbb{P}$ tal que $n<p<2 n$. Em particular,

$$
g_{n}<p_{n}, \forall n \in \mathbb{Z}_{+}
$$

Este teorema foi conjecturado por Joseph Bertrand em 1845 e provado pela primeira vez por Chebyshev em 1852. Recomendamos [7] , p. 9 - 12, para uma prova deste teorema. Entretanto, muito mais pode ser dito sobre um limitante superior de $g_{n}$. Um exemplo disso é o teorema dos números primos.

Teorema 5.1.3 (Hadamard-de la Vallée-Poussin, 1896). $\lim _{n \rightarrow \infty} \frac{p_{n}}{n \ln (n)}=1$.

Recomendamos [16] para uma prova deste teorema. Em particular,

$$
\lim _{n \rightarrow \infty} \frac{g_{n}}{p_{n}}=0 .
$$

Continuando nesta direção, o melhor resultado conhecido é o de BakerHarmar-Pintz.

Teorema 5.1.4 (Baker-Harman-Pintz, 2001). $g_{n} \leq p_{n}^{0.525}, \forall n \gg 0$.

Recomendamos [12] para uma prova desse teorema. Na seção seguinte, apresentaremos uma conjectura que supera significativamente qualquer estimativa conhecida sobre um limitante superior de $g_{n}$. Para encerrar esta seção, vamos comentar rapidamente sobre a quantidade $\lim \inf g_{n}$. Este é um problema em aberto na teoria dos números muito antigo, conhecido como conjectura dos primos gêmeos.

Problema 5.1.5. Existem infinitos primos gêmeos? Equivalentemente, é verdade que $\lim \inf g_{n}=2$ ?

Na verdade, por muito tempo ficou em aberto decidir se $\lim \inf g_{n}<\infty$, até que em 2013 Yitang Zhang demonstrou seu teorema.

Teorema 5.1.6 (Zhang, 2013). $\liminf g_{n}<7 \cdot 10^{7}$.

Recomendamos [87] para uma prova deste teorema. Mais recentemente ainda, vários matemáticos se uniram num projeto compartilhado na internet (projeto Polymath 8) para diminuir o limitante explícito no teorema de Zhang. O melhor limitante incondicional até agora é 246 (veja [65] para uma prova desta afirmação e para maiores informações sobre este limitante).

\subsection{A conjectura de Firoozbakht e algumas con- sequências}

O que faz um problema de matemática ser considerado bom? Para o autor desta tese, a resposta desta questão envolve três ingredientes principais: 
- É fácil de entender;

- É muito difícil de resolver;

- Apresenta muitas implicações fortes.

Um exemplo de um problema satisfazendo as três condições acima é a conjectura de Firoozbakht. Em 1982 (veja [1]), Farideh Firoozbakht, da Universidade de Isfahan, conjecturou o seguinte.

Conjectura 5.2.1 (Firoozbakht, 1982). Se $p_{n}$ for o n-ésimo número primo, então a sequência $\left\{\sqrt[n]{p_{n}}\right\}_{n \in \mathbb{Z}_{+}}$é estritamente decrescente.

Vejamos os primeiros casos:

$\begin{array}{ccc}n & p_{n} & \sqrt[n]{p_{n}} \\ 1 & 2 & 2,0000 \\ 2 & 3 & 1,7321 \\ 3 & 5 & 1,7100 \\ 4 & 7 & 1,6266 \\ 5 & 11 & 1,6154 \\ 6 & 13 & 1,5334 \\ 7 & 17 & 1,4989 \\ 8 & 19 & 1,4449 \\ 9 & 23 & 1,4168 \\ 10 & 29 & 1,4004\end{array}$

A conjectura de Firoozbakht já foi verificada para todos os primos abaixo de $4 \cdot 10^{18}$ (veja [40]), mas não existe consenso acerca do valor lógico desta conjectura. O autor desta tese, em particular, acredita que ela é verdadeira. A importância da conjectura de Firoozbakht é que ela é a mais ousada (e razoável) afirmação conhecida sobre prime gaps. Utilizando a função $g_{n}$, a conjectura de Firoozbakht pode ser reescrita da seguinte maneira:

$$
g_{n} \leq p_{n}\left(\sqrt[n]{p_{n}}-1\right), \forall n \in \mathbb{Z}_{+}
$$

O teorema 5.2.3 abaixo nos mostra quão forte é a conjectura de Firoozbakht com respeito aos limitantes superiores conhecidos atualmente sobre a função $g_{n}$. Para isto, precisamos de um lema preparatório.

Lema 5.2.2. $\frac{g_{n}}{g_{n}+p_{n}}<\ln \left(p_{n+1}\right)-\ln \left(p_{n}\right), \forall n \in \mathbb{Z}_{+}$.

Demonstração: Sabemos do cálculo que $1+\ln (x)<x, \forall x \in(0,1)$. Tomando

$$
x=\frac{p_{n}}{p_{n+1}},
$$

obtemos o resultado. 
Teorema 5.2.3. Se a conjectura de Firoozbakht for verdadeira, então

$$
g_{n}<\ln ^{2}\left(p_{n}\right)-\ln \left(p_{n}\right)-1, \forall n>9 .
$$

Em particular,

$$
g_{n}<\ln ^{2}\left(p_{n}\right)-\ln \left(p_{n}\right), \forall n>4, \quad e \quad \limsup _{n \rightarrow \infty} \frac{g_{n}}{\ln ^{2}\left(p_{n}\right)} \leq 1 .
$$

Demonstração: (Seguindo [41]) Suponha que $\sqrt[n]{p_{n}}>\sqrt[n+1]{p_{n+1}}, \forall n \in \mathbb{Z}_{+}$. Tomando o logaritmo natural, obtemos

$$
\frac{\ln \left(p_{n}\right)}{n}>\frac{\ln \left(p_{n+1}\right)}{n+1}, \forall n \in \mathbb{Z}_{+}
$$

Isolando $n$, obtemos

$$
n<\frac{\ln \left(p_{n}\right)}{\ln \left(p_{n+1}\right)-\ln \left(p_{n}\right)}, \forall n \in \mathbb{Z}_{+} .
$$

Utilizando o lema anterior, temos

$$
n<\ln \left(p_{n}\right)+\frac{\ln \left(p_{n}\right) \cdot p_{n}}{g_{n}}, \forall n \in \mathbb{Z}_{+} .
$$

Por outro lado, um cálculo nos mostra que

$$
\frac{x+\ln ^{2}(x)}{\ln (x)-1-\frac{1}{\ln (x)}}<\frac{x}{\ln (x)-1-\frac{1}{\ln (x)}-\frac{1}{\ln ^{2}(x)}}, \forall x \geq 285967 .
$$

Se $\pi(x)$ denota a função contadora dos números primos, então o corolário 3.6 de [11] afirma que

$$
\frac{x}{\ln (x)-1-\frac{1}{\ln (x)}-\frac{1}{\ln ^{2}(x)}}<\pi(x), \forall x \geq 1772201 .
$$

Dessa forma,

$$
\frac{x+\ln ^{2}(x)}{\ln (x)-1-\frac{1}{\ln (x)}}<\pi(x), \forall x \geq 1772201 .
$$

Tomando $x=p_{n}$ e lembrando que $1772201=p_{133115}$, temos

$$
\frac{p_{n}+\ln ^{2}\left(p_{n}\right)}{\ln \left(p_{n}\right)-1-\frac{1}{\ln \left(p_{n}\right)}}<\pi\left(p_{n}\right)=n<\ln \left(p_{n}\right)+\frac{\ln \left(p_{n}\right) \cdot p_{n}}{g_{n}}, \forall n \geq 133115 .
$$

Isolando $g_{n}$, temos

$$
g_{n}<\frac{p_{n}}{p_{n}+\ln \left(p_{n}\right)+1} \cdot\left[\ln ^{2}\left(p_{n}\right)-\ln \left(p_{n}\right)-1\right], \forall n \geq 133115 .
$$

Mas

$$
\frac{p_{n}}{p_{n}+\ln \left(p_{n}\right)+1}<1, \forall n \in \mathbb{Z}_{+}
$$

Portanto

$$
g_{n}<\ln ^{2}\left(p_{n}\right)-\ln \left(p_{n}\right)-1, \forall n \geq 133115 .
$$

Com a ajuda de um computador para checar os casos restantes, $(10 \leq n \leq 133114)$, terminamos a prova. 
Vale citar que se uma ligeira modificação for feita na tese do teorema 5.2.3, é possível obter uma condição suficiente para demonstrar a conjectura de Firoozbakht, conforme o seguinte teorema.

Teorema 5.2.4 (Kourbatov, 2015). Se

$$
g_{n}<\ln ^{2}\left(p_{n}\right)-\ln \left(p_{n}\right)-1,17, \forall n>9,
$$

então a conjectura de Firoozbakht é verdadeira.

Recomendamos [41] para uma prova deste teorema e para o leitor tomar conhecimento de outras condições suficientes para demonstrar a conjectura de Firoozbakht. Agora vamos ver como a conjectura de Firoozbakht, por meio do teorema 5.2 .3 , pode ser usada para resolver alguns problemas clássico sobre números primos ainda em aberto. O primeiro exemplo é a conjectura de Andrica (veja [8]).

Conjectura 5.2.5 (Andrica, 1986). $g_{n}<2 \sqrt{p_{n}}+1, \forall n \in \mathbb{Z}_{+}$.

Demonstração: Do cálculo, sabemos que

$$
\ln ^{2}(x)-\ln (x)-1<2 \sqrt{x}+1, \forall x \geq 1 .
$$

Assim, se a conjectura de Firoozbakht for verdadeira, então

$$
g_{n}<\ln ^{2}\left(p_{n}\right)-\ln \left(p_{n}\right)-1<2 \sqrt{p_{n}}+1, \forall n>9 .
$$

Verificando os casos restantes $(1 \leq n \leq 8)$, obtemos o resultado.

Outra bela consequência da conjectura de Firoozbakht é a conjectura de Oppermann (veja [62]).

Conjectura 5.2.6 (Oppermann, 1882). $\pi\left(n^{2}-n\right)<\pi\left(n^{2}\right)<\pi\left(n^{2}+n\right), \forall n \geq 2$.

Demonstração: A conjectura de Oppermann é verdadeira para $n \in\{2, \ldots, 74\}$. Agora suponha que exista $n \in \mathbb{N}, n \geq 75$, tal que $\pi\left(n^{2}-n\right)=\pi\left(n^{2}\right)$, e seja

$$
m=\pi\left(n^{2}-n\right) \text {. }
$$

Então

$$
p_{m}<n^{2}-n<n^{2}<p_{m+1}
$$

Portanto

$$
g_{m}=p_{m+1}-p_{m}>n
$$

o que implica

$$
n<g_{m}<\ln ^{2}\left(p_{m}\right)-\ln \left(p_{m}\right)-1<\ln ^{2}\left(p_{m}\right)<\ln ^{2}\left(n^{2}-n\right) .
$$

Mas, do cálculo, sabemos que

$$
x>\ln ^{2}\left(x^{2}+x\right), \forall x \geq 75 .
$$

Isso é um absurdo. Logo

$$
\pi\left(n^{2}-n\right)<\pi\left(n^{2}\right)
$$

Um argumento similar para a outra desigualdade termina a prova. 
Uma consequência direta da conjectura de Oppermann é a conjectura de Legendre (veja [2]).

Conjectura 5.2.7 (Legendre, forma forte). Existem pelo menos dois primos entre dois quadrados consecutivos.

A conjectura de Legendre, por sua vez ${ }^{1}$, implica a conjectura de Brocard (veja [3]), assim como a conjectura de Legendre para cubos.

Conjectura 5.2.8 (Brocard). Existem pelo menos quatro números primos entre $p_{n}{ }^{2}$ $e p_{n+1}^{2}, \forall n \geq 2$.

Conjectura 5.2.9 (Conjectura de Legendre para cubos, forma forte). Existem pelo menos quatro números primos entre dois cubos consecutivos.

Para encerrar esta seção, apresentamos duas conjecturas bastante recentes, relacionadas com a conjectura de Firoozbakht (veja [4]).

Conjectura 5.2.10 (Nicholson, 2013). $\left(\frac{p_{n+1}}{p_{n}}\right)^{n}<n \ln (n), \forall n \geq 5$.

Conjectura 5.2.11 (Forgues, 2014). $\left(\frac{\ln \left(p_{n+1}\right)}{\ln \left(p_{n}\right)}\right)^{n}<e, \forall n \in \mathbb{Z}_{+}$.

Para ver como estas conjecturas se relacionam com a conjectura de Firoozbakht, vamos lembrar o teorema de Rosser.

Teorema 5.2.12 (Rosser, 1939). $p_{n}>n \ln (n), \forall n \in \mathbb{Z}_{+}$.

Recomendamos [69] para uma prova deste teorema.

Teorema 5.2.13. Nicholson $\Rightarrow$ Firoozbakht $\Rightarrow$ Forgues.

Demonstração: A conjectura de Nicholson é a afirmação

$$
\left(\frac{p_{n+1}}{p_{n}}\right)^{n}<n \ln (n), \forall n \geq 5 .
$$

Pelo teorema de Rosser,

$$
\left(\frac{p_{n+1}}{p_{n}}\right)^{n}<p_{n}, \forall n \in \mathbb{Z}_{+}
$$

o que é equivalente a

$$
p_{n+1}{ }^{n}<p_{n}{ }^{n+1}, \forall n \in \mathbb{Z}_{+},
$$

o que, por sua vez, é equivalente à conjectura de Firoozbakht. Agora, tomando logaritmos na última inequação, obtemos

$$
\frac{\ln \left(p_{n+1}\right)}{\ln \left(p_{n}\right)}<1+\frac{1}{n}
$$

Elevando à $n$-ésima potência, obtemos

$$
\left(\frac{\ln \left(p_{n+1}\right)}{\ln \left(p_{n}\right)}\right)^{n}<\left(1+\frac{1}{n}\right)^{n}<e, \forall n \in \mathbb{Z}_{+},
$$

que é a conjectura de Forgues.

\footnotetext{
${ }^{1}$ Lembre que $p_{n+1} \geq p_{n}+2, \forall n \geq 2$.
} 


\subsection{Uma consequência do teorema de Zhang}

Seja $k$ um inteiro positivo par. Denotaremos

$$
Y_{k}=\left\{n \in \mathbb{Z}_{+} ; g_{n}=k\right\}
$$

para simplificar a notação.

Teorema 5.3.1. Seja $k$ um inteiro positivo par. Se $Y_{k}$ for infinito, então

$$
\sqrt[n]{p_{n}}>\sqrt[n+1]{p_{n+1}}, \forall n \in Y_{k}, n \gg 0 .
$$

Em particular, a conjectura de Firoozbakht é verdadeira para infinitos $n \in \mathbb{Z}_{+}$.

Demonstração: Pelo teorema 5.1.6, sabemos que algum $Y_{k}$ é infinito. Portanto, seja $k$ um inteiro positivo par tal que $Y_{k}$ é infinito e seja $n \in Y_{k}$. Então

$$
\frac{p_{n}{ }^{n+1}}{p_{n+1}{ }^{n}}=\frac{p_{n}{ }^{n+1}}{\left(p_{n}+k\right)^{n}}=\left(\frac{p_{n}}{p_{n}+k}\right)^{n} \cdot p_{n}=\left[\left(\frac{1}{1+\frac{k}{p_{n}}}\right)^{\frac{p_{n}}{k}}\right]^{\frac{k n}{p_{n}}} \cdot p_{n}
$$

Como

$$
\lim _{x \rightarrow \infty}\left(\frac{1}{1+\frac{1}{x}}\right)^{x}=\frac{1}{e}
$$

e

$$
\lim _{n \rightarrow \infty} \frac{k n}{p_{n}}=0
$$

(pelo teorema 5.1.3), então

$$
\left[\left(\frac{1}{1+\frac{k}{p_{n}}}\right)^{\frac{p_{n}}{k}}\right]^{\frac{k n}{p_{n}}} \rightarrow 1 \text { se } n \rightarrow \infty, n \in Y_{k} .
$$

Logo

$$
\lim _{\substack{n \rightarrow \infty \\ n \in Y_{k}}} \frac{p_{n}{ }^{n+1}}{p_{n+1}{ }^{n}}=\infty
$$

Em particular,

$$
p_{n}{ }^{n+1}>p_{n+1}{ }^{n}, \forall n \in Y_{k}, n \gg 0 .
$$

Isto é equivalente ao enunciado do teorema.

Para finalizar, vale ainda comentar sobre o atual estado do relacionamento da conjectura de Firoozbakht com dois outros problemas bastante famosos em teoria dos números: a hipótese de Riemann e a conjectura de Cramér.

Cramér mostrou que a hipótese de Riemann implica $g_{n} \ll \sqrt{p_{n}} \ln \left(p_{n}\right)$. Recomendamos [18] ou [19] para uma prova deste teorema. Ou seja, mesmo a hipótese de Riemann, um dos problemas mais celebrados da atualidade, implica uma ordem de crescimento da função $g_{n}$ bem maior do que uma ordem de crescimento predita pela conjectura de Firoozbakht, que é $\ln ^{2}\left(p_{n}\right)$. Entretanto, vale citar que o autor 
desta tese desconhece se a hipótese de Riemann implica uma ordem de crescimento da função $g_{n}$ melhor do que $\sqrt{p_{n}} \ln \left(p_{n}\right)$.

Já a conjectura de Cramér é a afirmação $g_{n} \ll \ln ^{2}\left(p_{n}\right)$. É claro que a conjectura de Firoozbakht implica a conjectura de Cramér, conforme o teorema 5.2.3. Cramér apresentou sua conjectura baseado em um modelo probabilístico dos números primos. Neste modelo, Cramér mostrou que a relação

$$
\limsup _{n \rightarrow \infty} \frac{g_{n}}{\ln ^{2}\left(p_{n}\right)}=1
$$

é verdadeira com probabilidade 1 (veja [19]). Entretanto, como bem apontou Andrew Granville em [32], o teorema de Maier mostra que o modelo de Cramér não descreve adequadamente a distribuição dos números primos em intervalos pequenos, e que um refinamento do modelo de Cramér levando em conta a divisibilidade por números primos pequenos levaria à relação

$$
\limsup _{n \rightarrow \infty} \frac{g_{n}}{\ln ^{2}\left(p_{n}\right)} \geq 2 e^{-\gamma} \approx 1,1229 \ldots
$$

onde

$$
\gamma=\lim _{n \rightarrow \infty}\left(-\ln (n)+\sum_{k=1}^{n} \frac{1}{k}\right) \approx 0,5772 \ldots
$$

é a constante de Euler-Mascheroni. Mais especificamente, se $\pi(x)$ denota a função contadora dos números primos, então o modelo de Cramér prediz que

$$
\lim _{n \rightarrow \infty} \frac{\pi\left(x+(\ln (x))^{\lambda}\right)-\pi(x)}{(\ln (x))^{\lambda-1}}=1, \forall \lambda \geq 2,
$$

enquanto que o teorema de Maier afirma que se $\lambda>1$, então

$$
\liminf _{n \rightarrow \infty} \frac{\pi\left(x+(\ln (x))^{\lambda}\right)-\pi(x)}{(\ln (x))^{\lambda-1}}<1 \quad \text { e } \quad \limsup _{n \rightarrow \infty} \frac{\pi\left(x+(\ln (x))^{\lambda}\right)-\pi(x)}{(\ln (x))^{\lambda-1}}>1 .
$$

Recomendamos [53] para uma prova do teorema de Maier. Tudo isso mostra o quão importante é um estudo aprofundado da conjectura de Firoozbakht, ou, mais geralmente, da função $g_{n}$. 


\section{Capítulo 6}

\section{Aplicações da teoria algébrica dos números aos prime gaps}

Este capítulo apresenta uma abordagem diferente da usual para tratar do problema dos prime gaps, tomando como base a conjectura de Firoozbakht. Normalmente, as técnicas utilizadas para estudar a função $g_{n}$ têm origem na análise, como crivos, análise combinatória ou estimativas de certas integrais. Neste capítulo, vamos estudar a conjectura de Firoozbakht de um ponto de vista mais algébrico, utilizando a teoria algébrica dos números e a teoria de Galois como pano de fundo para desenvolver o nosso estudo. Para isto, vamos inicialmente apresentar alguns resultados destas duas belas áreas da matemática que serão úteis no desenvolvimento de nossa abordagem. Embora não citando uma referência para cada resultado da próxima seção, todas as provas dos teoremas da próxima seção podem ser encontradas nos livros [5], [17], [25], [39], [55], [56], [58], [59], [61], [66], [70], [72], [75], [76] e [83]. A escolha de fazer estas referências desta forma reflete o fato de que o autor desta tese não acredita que haja uma melhor prova para cada um dos resultados da próxima seção em algum livro específico, dada a excelente qualidade de cada uma das referências citadas. A segunda seção se encarrega de apresentar a abordagem algébrica da conjectura de Firoozbakht.

\subsection{Background da teoria algébrica dos números}

Definição 6.1.1. Um corpo de números é uma extensão finita de $\mathbb{Q}$.

Definição 6.1.2. Dizemos que a $\in \mathbb{C}$ é um número algébrico se existe um polinômio mônico não nulo $f(x) \in \mathbb{Q}[x]$ tal que $f(a)=0$. Denotamos o conjunto de todos os números algébricos por $\mathbb{A}$. Um número não algébrico é dito ser transcendente.

É claro que todo número racional é algébrico, mas a recíproca não é verdadeira: $\sqrt{2} \in \mathbb{A}-\mathbb{Q}$. Como existe apenas uma quantidade enumerável de números algébricos, existem números transcendentes. Mais especificamente, temos o seguinte resultado. 
Teorema 6.1.3 (Lindemann, 1882). $\pi$ é transcendente.

Assim, embora a soma de dois números transcendentes possa não ser transcendente $^{1}$, isso não acontece com os números algébricos.

Teorema 6.1.4. $\mathbb{A}$ é um corpo.

Outra noção útil é o conceito de inteiro algébrico.

Definição 6.1.5. Dizemos que $a \in \mathbb{C}$ é um inteiro algébrico se existe um polinômio mônico não nulo $f(x) \in \mathbb{Z}[x]$ tal que $f(a)=0$. Denotamos o conjunto de todos os inteiros algébricos por $\mathcal{O}$.

Teorema 6.1.6. $\mathcal{O}$ é um dominio de integridade.

Definição 6.1.7. Seja $K$ um corpo de números. Definimos o anel de números de $K$ como sendo o conjunto $\mathcal{O}_{K}=\mathcal{O} \cap K$.

Como a interseção de uma família de domínios de integridade é um domínio de integridade, então $\mathcal{O}_{K}$ é um domínio de integridade, qualquer que seja o corpo de números $K$. Este domínio de integridade tem boas propriedades.

Definição 6.1.8. Dizemos que um domínio de integridade $D$ é um domínio de Dedekind se $D$ for integralmente fechado, noetheriano e se todo ideal primo não nulo for maximal.

Teorema 6.1.9. Se $K$ for um corpo de números, então $\mathcal{O}_{K}$ é um domínio de Dedekind.

Outra boa propriedade de $\mathcal{O}_{K}$ é que ele admite uma base integral sobre $\mathbb{Z}$.

Teorema 6.1.10. Seja $K$ corpo de números de grau $n$ sobre $\mathbb{Q}$. Então $\mathcal{O}_{K}$ é um grupo abeliano livre de ordem n. Equivalentemente, existem $\beta_{1}, \ldots, \beta_{n} \in \mathcal{O}_{K}$ tais que todo $\alpha \in \mathcal{O}_{K}$ se escreve de maneira única como

$$
\alpha=m_{1} \beta_{1}+\cdots+m_{n} \beta_{n}
$$

onde $m_{1}, \ldots, m_{n} \in \mathbb{Z}$. Neste caso, dizemos que o conjunto $\mathcal{B}=\left\{\beta_{1}, \ldots, \beta_{n}\right\}$ é uma base integral de $\mathcal{O}_{K}$.

Pode-se mostrar que toda base integral de $\mathcal{O}_{K}$ é uma base de $K$ sobre $\mathbb{Q}$. Em particular, $\operatorname{disc}(\mathcal{B}) \neq 0$.

Definição 6.1.11. Seja $K$ corpo de números de grau $n$ sobre $\mathbb{Q}$ e sejam $\sigma_{1}, \ldots, \sigma_{n}$ os $n$ mergulhos de $K$ em $\mathbb{C}$. Se $\mathcal{B}=\left\{\beta_{1}, \ldots, \beta_{n}\right\}$ for uma base integral de $\mathcal{O}_{K}$, definimos o discriminante de $\mathcal{B}$ por

$$
\operatorname{disc}(\mathcal{B})=\left(\operatorname{det}\left(\sigma_{i}\left(\beta_{j}\right)\right)\right)^{2} .
$$

\footnotetext{
${ }^{1}$ Basta tomar $\pi+(-\pi)=0$.
} 
Sobre bases integrais temos o seguinte resultado.

Teorema 6.1.12. Se $\mathcal{B}_{1}$ e $\mathcal{B}_{2}$ forem duas bases integrais do anel de números $\mathcal{O}_{K}$, então $\operatorname{disc}\left(\mathcal{B}_{1}\right)=\operatorname{disc}\left(\mathcal{B}_{2}\right)$.

Assim, o discriminante de uma base integral é um invariante do corpo $K$.

Definição 6.1.13. Sejam $K$ um corpo de números e $\mathcal{B}$ uma base de seu anel de números $\mathcal{O}_{K}$. Definimos os discriminante de $K$ por $\operatorname{disc}(K)=\operatorname{disc}(\mathcal{B})$.

A noção de discriminante será usada fortemente na próxima seção, por meio do seguinte teorema de Minkowski.

Teorema 6.1.14 (Minkowski). Seja $K$ corpo de números. Se $|\operatorname{disc}(K)|=1$, então $K=\mathbb{Q}$.

Para uma torre de corpos de números, temos o seguinte resultado.

Teorema 6.1.15. Sejam $K$ e $L$ corpos de números tais que $K \subseteq L$. Então $\operatorname{disc}(K)$ divide $\operatorname{disc}(L)$.

Outro conceito que será usado será a noção de ramificação.

Definição 6.1.16. Seja $K$ um corpo de números. Dizemos que um número primo $p \in \mathbb{P}$ ramifica em $K$ se $p$ divide $\operatorname{disc}(K)$.

Vale notar que esta não é a definição usual de ramificação, mas é equivalente à usual. Em particular, segue do teorema de Minkowski o seguinte corolário.

Corolário 6.1.17. Seja $K$ um corpo de números. Se $K \neq \mathbb{Q}$, então algum primo $p \in \mathbb{P}$ ramifica em $K$.

Corolário 6.1.18. Sejam $K$ e $L$ dois corpos de números. $\operatorname{Se} \operatorname{mdc}(\operatorname{disc}(K), \operatorname{disc}(L))=$ 1 , então $K \cap L=\mathbb{Q}$.

Demonstração: Seja $M=K \cap L$. Se $M \neq \mathbb{Q}$, então existe $p \in \mathbb{P}$ tal que $p$ divide $\operatorname{disc}(M)$. Como $M \subseteq K$ e $M \subseteq L$, então $p$ divide $\operatorname{disc}(K)$ e $p$ divide $\operatorname{disc}(L)$, absurdo.

Teorema 6.1.19. Sejam $K$ e $L$ duas extensões galoisianas de $\mathbb{Q}$. Se $K \cap L=\mathbb{Q}$, então

$$
\operatorname{Gal}(K L) \simeq \operatorname{Gal}(K) \times \operatorname{Gal}(L) .
$$

Teorema 6.1.20. Seja $n \in \mathbb{Z}, n \geq 2$. Então

$$
\operatorname{Gal}\left(x^{n}-p_{n}, \mathbb{Q}\right) \simeq\left(\frac{\mathbb{Z}}{n \mathbb{Z}}\right) \rtimes\left(\frac{\mathbb{Z}}{n \mathbb{Z}}\right)^{\times} .
$$

Para este último resultado, veja em particular, [45], capítulo 6, seção 9, e [85], capítulo 4, seção 6. Com estes conceitos desenvolvidos, podemos apresentar um estudo alternativo da conjectura de Firoozbakht. 


\subsection{Estudo do número $a_{n}$}

A conjectura de Firoozbakht afirma que

$$
\sqrt[n]{p_{n}}>\sqrt[n+1]{p_{n+1}}, \forall n \in \mathbb{Z}_{+}
$$

Equivalentemente, ela pode ser escrita na forma

$$
\sqrt[n]{p_{n}}-\sqrt[n+1]{p_{n+1}}>0, \forall n \in \mathbb{Z}_{+} .
$$

Assim, é natural perguntar: que tipo de número é $a_{n}=\sqrt[n]{p_{n}}-\sqrt[n+1]{p_{n+1}}$ ?

Este número é um inteiro algébrico, pois é a diferença de dois inteiros algébricos. Assim, seu polinômio minimal sobre $\mathbb{Q}$ tem coeficientes inteiros. A ideia que propomos aqui é tentar obter informações a respeito de $a_{n}$ estudando seu polinômio minimal $f_{n}(x)$. Note que, com esta abordagem, temos a possibilidade de usar todas as ferramentas algébricas existentes. A primeira ação que tomaremos para determinar qual é o polinômio minimal de $a_{n}$ será descrever seus conjugados algébricos.

Para isto, fixe ${ }^{2} n \in \mathbb{Z}, n \geq 2$, e seja

$$
K_{n}=\mathbb{Q}\left(\sqrt[n]{p_{n}}, \xi_{n}\right)
$$

o corpo de fatoração de $x^{n}-p_{n}$. Então a extensão $K_{n} / \mathbb{Q}$ é galoisiana e tem discriminante

$$
\operatorname{disc}\left(K_{n}\right)=\left\{\begin{array}{ccc}
n^{n} p_{n}{ }^{n-1}, & \text { se } n \equiv 1,2 \quad(\bmod 4) \\
-n^{n} p_{n}{ }^{n-1}, & \text { se } n \equiv 3,4 \quad(\bmod 4)
\end{array}\right.
$$
Como

Sabemos que os conjugados algébricos de $\sqrt[n]{p_{n}}$ são $\xi_{n} \sqrt[i]{p_{n}}$, onde $i \in \overleftarrow{n}$.

$$
\mathbb{Q}\left(\sqrt[n]{p_{n}}\right) \cap \mathbb{Q}\left(\xi_{n}\right)=\mathbb{Q}
$$

(veja [85], capítulo 4, seção 6), então

$$
\left[K_{n}: \mathbb{Q}\right]=n \varphi(n) .
$$

Agora note que

$$
\operatorname{mdc}\left(\operatorname{disc}\left(K_{n}\right), \operatorname{disc}\left(K_{n+1}\right)\right)=1 .
$$

Pelos resultados da seção anterior,

- $K_{n} K_{n+1}$ é uma extensão galoisiana de $\mathbb{Q}$;

- $\left[K_{n} K_{n+1}: \mathbb{Q}\right]=n(n+1) \varphi(n) \varphi(n+1)$;

- $\operatorname{Gal}\left(K_{n} K_{n+1} / \mathbb{Q}\right) \simeq\left(\left(\frac{\mathbb{Z}}{n \mathbb{Z}}\right) \rtimes\left(\frac{\mathbb{Z}}{n \mathbb{Z}}\right)^{\times}\right) \times\left(\left(\frac{\mathbb{Z}}{(n+1) \mathbb{Z}}\right) \rtimes\left(\frac{\mathbb{Z}}{(n+1) \mathbb{Z}}\right)^{\times}\right)$

- os conjugados algébricos de $a_{n}$ são

$$
r_{i, j, n}=\xi_{n} \sqrt[i]{p_{n}}-\xi_{n+1} \sqrt[j]{\sqrt[n+1]{p_{n+1}}}, \quad i \in \overleftarrow{n}, j \in \overleftarrow{n+1}
$$

\footnotetext{
${ }^{2} \mathrm{O}$ caso $n=1$ é degenerado: $\sqrt[1]{2}=2 \in \mathbb{Z}$.
} 
Em particular, o grau de $f_{n}(x)$ é $n(n+1)$ e podemos calcular os coeficientes de $f_{n}(x)$ pelas relações de Girard. Em todo o argumento abaixo, devemos lembrar que, como $a_{n}$ é um inteiro algébrico, os coeficientes de $f_{n}$ são números inteiros. Também é claro que o coeficiente dominante de $f_{n}(x)$ é 1 . Escrevamos de início

$$
f_{n}(x)=\sum_{i=0}^{n(n+1)} \lambda_{n, i} x^{i} .
$$

Teorema 6.2.1. Seja $i \in\{1, \ldots, n(n+1)-1\}$. Então $\lambda_{i, n} \neq 0$ se, e somente se, a equação

$$
r n+s(n+1)=n(n+1)-i
$$

tem uma única solução com $r, s \in \mathbb{N}$. Neste caso,

$$
\lambda_{n, i}=\theta_{n, i} p_{n}^{r} p_{n+1}^{s},
$$

onde $\theta_{n, i}$ é um número inteiro obtido a partir de uma combinação linear de

$$
\left\{\xi_{n}{ }^{i^{\prime}} \xi_{n+1}{ }^{j^{\prime}} ; i^{\prime} \in \overleftarrow{n}, j^{\prime} \in \overleftarrow{n+1}\right\}
$$

Demonstração: De fato, fixe $i \in \overleftarrow{n(n+1)-1}$. Então

$$
\lambda_{n, i}=\sum_{\substack{A \subseteq \tilde{n} \times \overleftarrow{n+1} \\ \#(A)=n(n+1)-i}}\left(\prod_{(i, j) \in A} r_{i, j, n}\right) .
$$

Como $\lambda_{n, i} \in \mathbb{Z}$, então $\lambda_{n, i}$ é simplesmente a soma das coordenadas inteiras de cada um dos produtos

$$
\prod_{(i, j) \in A} r_{i, j, n}
$$

Para calcular estas coordenadas, vamos fixar

$$
A \subseteq \overleftarrow{n} \times \overleftarrow{n+1}
$$

tal que $\#(A)=n(n+1)-i$. Então

$$
\prod_{(i, j) \in A} r_{i, j, n}
$$

é uma soma de termos da forma

$$
\xi_{n} \sqrt[i^{\prime}]{p_{n}} i^{i^{\prime \prime}} \xi_{n+1} \sqrt[j^{\prime}]{\sqrt[n+1]{p_{n+1}}} j^{\prime \prime},
$$

onde

$$
i^{\prime \prime}+j^{\prime \prime}=n(n+1)-i .
$$

Como basta somar as coordenadas inteiras destes números, temos

$$
\xi_{n} \sqrt[i^{\prime}]{n_{p_{n}}} i^{i^{\prime \prime}} \xi_{n+1} \sqrt[j^{\prime}]{\sqrt[n+1]{p_{n+1}}} j^{j^{\prime \prime}} \in \mathbb{Z}
$$

se, e somente se

$$
i^{\prime \prime}=r n, \quad j^{\prime \prime}=s(n+1) \quad \text { e } \quad \xi_{n}{ }^{i^{\prime}} \xi_{n+1}{ }^{j^{\prime}} \in \mathbb{Z} .
$$

Dessa forma, 
- se a equação

$$
r n+s(n+1)=n(n+1)-i
$$

tem solução com $r, s \in \mathbb{N}$, então $\lambda_{n, i} \in \mathbb{Z}$ e é da forma

$$
\lambda_{n, i}=\theta_{n, i} p_{n}^{r} p_{n+1}^{s},
$$

onde $\theta_{n, i}$ é um número inteiro obtido a partir de uma combinação linear de

$$
\left\{\xi_{n}{ }^{i^{\prime}} \xi_{n+1}^{j^{\prime}} ; i^{\prime} \in \overleftarrow{n}, j^{\prime} \in \overleftarrow{n+1}\right\}
$$

Podemos olhar $\lambda_{n, i}$, neste caso, como sendo um produto obtido por meio de três contribuições: uma do corpo $\mathbb{Q}\left(\sqrt[n]{p_{n}}\right)$, a saber, $p_{n}{ }^{r} ;$ uma do corpo $\mathbb{Q}\left(\sqrt[n+1]{p_{n+1}}\right)$, a saber, $p_{n+1}{ }^{s}$; e uma do corpo ciclotômico $\mathbb{Q}\left(\xi_{n} \xi_{n+1}\right)=\mathbb{Q}\left(\xi_{n(n+1)}\right)$, a saber, $\theta_{n, i}$.

- se a equação

$$
r n+s(n+1)=n(n+1)-i
$$

não tem solução com $r, s \in \mathbb{N}$, como $\lambda_{n, i} \in \mathbb{Z}$, então $\theta_{n, i}=0$, o que implica $\lambda_{n, i}=0$.

Para verificar a unicidade, suponha que

$$
r n+s(n+1)=r^{\prime} n+s^{\prime}(n+1),
$$

com $0 \leq r, r^{\prime}<n+1$ e $0 \leq s, s^{\prime}<n$, pois $i \in \overleftarrow{n(n+1)-1}$. Então

$$
\left(r-r^{\prime}\right) n=\left(s^{\prime}-s\right)(n+1) .
$$

Como $\operatorname{mdc}(n, n+1)=1$, então $n$ divide $s^{\prime}-s$ e $r$ divide $r-r^{\prime}$, o que implica $r=r^{\prime}$ e $s=s^{\prime}$.

Teorema 6.2.2. $\lambda_{n, 0}=(-1)^{n+1}\left(p_{n}^{n+1}-p_{n+1}{ }^{n}\right)$.

Demonstração: Com efeito, sabemos que

$$
\lambda_{n, 0}=(-1)^{n(n+1)} \prod_{(i, j) \in \overleftarrow{n} \times \overleftarrow{n+1}} r_{i, j, n}=\prod_{(i, j) \in \overleftarrow{n} \times \overleftarrow{n+1}} r_{i, j, n}
$$

e que $\lambda_{n, 0} \in \mathbb{Z}$. Raciocinando da mesma forma que antes, este produto é uma soma de termos da forma

$$
\xi_{n} \sqrt[i^{\prime}]{p_{n}} i^{i^{\prime \prime}} \xi_{n+1} \sqrt[j^{\prime}]{\sqrt[n+1]{p_{n+1}}} j^{\prime \prime}
$$

onde

$$
i^{\prime \prime}+j^{\prime \prime}=n(n+1) .
$$

Como basta somar as coordenadas inteiras destes números e como $\lambda_{n, 0} \in \mathbb{Z}$, então

$$
i^{\prime \prime}=r n, \quad j^{\prime \prime}=s(n+1) \quad \text { e } \quad \xi_{n}{ }^{i^{\prime}} \xi_{n+1}{ }^{j^{\prime}} \in \mathbb{Z} \text {. }
$$


Mas aqui temos uma diferença. Como $i \in \overleftarrow{n}$ e $j \in \overleftarrow{n+1}$, então temos apenas duas opções: $r=n+1$ e $s=n$. Quando $r=n+1$, então $s=0$ e portanto

$$
\begin{aligned}
\xi_{n} \sqrt[i^{\prime}]{p_{n}} i^{i^{\prime \prime}} \xi_{n+1} \sqrt[j^{\prime}]{\sqrt[n+1]{p_{n+1}} j^{\prime \prime}} & =\prod_{j=1}^{n+1}\left(\prod_{i=1}^{n}\left(\xi_{n} \sqrt[i]{p_{n}}\right)\right) \\
& =\prod_{j=1}^{n+1}\left((-1)^{n+1} p_{n}\right)=(-1)^{n+1} p_{n}{ }^{n+1}
\end{aligned}
$$

Quando $s=n$, então $r=0$ e portanto

$$
\begin{aligned}
\xi_{n} i^{\prime} \sqrt[n]{p_{n}} i^{\prime \prime} \xi_{n+1} \sqrt[j^{\prime}]{\sqrt[n+1]{p_{n+1}}} j^{\prime \prime} & =\prod_{i=1}^{n}\left(\prod_{j=1}^{n+1}\left(\xi_{n+1} \sqrt[j+1]{p_{n+1}}\right)\right) \\
& =\prod_{i=1}^{n}\left((-1)^{n+2} p_{n+1}\right)=(-1)^{n+2} p_{n+1}^{n}
\end{aligned}
$$

o que implica

$$
\lambda_{n, 0}=(-1)^{n+1} p_{n}{ }^{n+1}+(-1)^{n+2} p_{n+1}^{n}=(-1)^{n+1}\left(p_{n}{ }^{n+1}-p_{n+1}{ }^{n}\right) .
$$

Por exemplo, os primeiros $f_{n}(x)$ são

$f_{1}(x)=x^{2}-4 x+1$

$f_{2}(x)=x^{6}-9 x^{4}+10 x^{3}+27 x^{2}+90 x-2$

$f_{3}(x)=x^{12}-20 x^{9}-21 x^{8}+150 x^{6}-1680 x^{5}+147 x^{4}-500 x^{3}-5250 x^{2}-2940 x+282$.

Corolário 6.2.3. Seja $n \in \mathbb{N}$. Então

$$
\sqrt[n]{p_{n}}>\sqrt[n+1]{p_{n+1}}
$$

se, e somente se,

$$
\operatorname{sgn}\left(f_{n}(0)\right)=(-1)^{n+1}
$$

Dessa forma, a conjectura de Firoozbakht é equivalente a saber o sinal do termo independente do polinômio $f_{n}(x)$. Isso permite uma fatoração do problema, dividindo um pouco a dificuldade de uma questão totalmente analítica (a conjectura de Firoozbakht é um problema acerca do sinal de uma desigualdade) com a álgebra. Encerramos esta seção com o seguinte resultado.

Teorema 6.2.4. Os polinômios $f_{n}(x)$ têm exatamente duas raízes reais. Mais especificamente,

- se $n$ for par, então as únicas duas raízes reais de $f_{n}(x)$ são $\pm \sqrt[n]{p_{n}}-\sqrt[n+1]{p_{n+1}}$;

- se $n$ for ímpar, então as únicas duas raízes reais de $f_{n}(x)$ são $\sqrt[n]{p_{n}} \pm \sqrt[n+1]{p_{n+1}}$. 
Demonstração: Seja $r_{i, j, n}$ uma raiz real de $f_{n}(x)$. Então $r_{i, j, n}=\overline{r_{i, j, n}}$, ou seja,

$$
\xi_{n} \sqrt[i]{p_{n}}-\xi_{n+1} \sqrt[j]{\sqrt[n+1]{p_{n+1}}}=\overline{\xi_{n}} \sqrt[n]{p_{n}}-\overline{\xi_{n+1}} \sqrt[n+1]{p_{n+1}}
$$

o que implica

$$
\left(\xi_{n}{ }^{i}-\overline{\xi_{n}}\right) \sqrt[n]{p_{n}}=\left(\xi_{n+1}^{j}-\overline{\xi_{n+1}^{j}}\right) \sqrt[n+1]{p_{n+1}} .
$$

Mas $K_{n} \cap K_{n+1}=\mathbb{Q}$ (ainda por [85], capítulo 4, seção 6), portanto

$$
\left(\xi_{n}{ }^{i}-\overline{\xi_{n}}\right) \sqrt[n]{p_{n}} \in \mathbb{Q} \quad \text { e } \quad\left(\xi_{n+1}^{j}-\overline{\xi_{n+1}^{j}}\right) \sqrt[n+1]{p_{n+1}} \in \mathbb{Q} .
$$

Mas

$$
\xi_{n}{ }^{i}-\overline{\xi_{n}}{ }^{i}=2 \xi_{4} \operatorname{sen}\left(\frac{2 \pi i}{n}\right) \quad \text { e } \quad \xi_{n+1}^{j}-\overline{\xi_{n+1}}{ }^{j}=2 \xi_{4} \operatorname{sen}\left(\frac{2 \pi j}{n+1}\right) .
$$

Logo sen $\left(\frac{2 \pi i}{n}\right)=0$ e sen $\left(\frac{2 \pi j}{n+1}\right)=0$. Assim,

- se $n$ for par, então $i \in\left\{\frac{n}{2}, n\right\}$ e $j=n+1$, o que nos leva às raízes

$$
\pm \sqrt[n]{p_{n}}-\sqrt[n+1]{p_{n+1}}
$$

- Já se $n$ for ímpar, então $i=n$ e $j \in\left\{\frac{n+1}{2}, n+1\right\}$, o que nos leva às raízes

$$
\sqrt[n]{p_{n}} \pm \sqrt[n+1]{p_{n+1}}
$$




\section{Trabalhos futuros}

Note que uma estratégia para resolver o problema de Firoozbakht pode ser a seguinte: fixado $n \in \mathbb{Z}, n \geq 2$, mostrar que existe $b_{n} \in \mathbb{R}$ tal que $\left|b_{n}\right|<\left|a_{n}\right|$ e $\operatorname{sgn}\left(f_{n}\left(b_{n}\right)\right)=(-1)^{n+1}$. Isto implicaria Firoozbakht.

Mas também pode ser o caso de que $f_{n}(x)$ não seja o polinômio mais adequado a ser usado para se estudar este problema utilizando este viés algébrico. Uma alternativa seria utilizar o polinômio

$$
g_{n}(x)=\left(x^{n}-p_{n}\right)^{n+1}+\left(x^{n+1}-p_{n+1}\right)^{n},
$$

que é um polinômio mônico em $\mathbb{Z}[x]$. Note que

$$
g_{n}(0)=(-1)^{n+1}\left(p_{n}{ }^{n+1}-p_{n+1}^{n}\right)=\lambda_{n, 0} .
$$

Outra possibilidade é perturbar os polinômios $x^{n}-p_{n}$ e $x^{n+1}-p_{n+1}$ para tentar transferir mais ainda a dificuldade de um problema analítico para a álgebra. Note que o que queremos saber é se a diferença das raízes positivas dos polinômios $x^{n}-p_{n}$ e $x^{n+1}-p_{n+1}$ é positiva. Poderíamos tomar os polinômios

$$
x^{n}-x-p_{n} \quad \text { e } \quad x^{n+1}-x-p_{n+1}
$$

e tentar reproduzir o raciocínio da seção anterior. Devido a esta leve perturbação, pode ser que seja mais fácil algebricamente descobrir o sinal do termo independente do polinômio $f_{n}(x)$ respectivo, e a partir daí restaria apenas mostrar que esta leve perturbação não altera o problema original, ainda deixando-o equivalente à conjectura de Firoozbakht.

Vale citar aqui que recentemente muitas perguntas "à la" conjectura de Firoozbakht começaram a ser feitas, muitas delas ainda sem resposta. Mais rigorosamente, dada uma sequência de números reais positivos $\left\{\gamma_{n}\right\}_{n \in \mathbb{Z}_{+}}$, a sequência $\left\{\sqrt[n]{\gamma_{n}}\right\}_{n \in \mathbb{Z}_{+}}$é decrescente (ou crescente)? Normalmente, a sequência $\left\{\gamma_{n}\right\}_{n \in \mathbb{Z}_{+}}$é alguma sequência muito conhecida, como a sequência dos números primos ou a sequência de Fibonacci. Para esta última, o problema foi resolvido ${ }^{3}$ só em 2012, utilizando métodos totalmente analíticos (veja [38] e [82]). Isso não só mostra o caráter recente destes questionamentos, quanto mostra importância de novos e variados métodos para resolver este tipo de problema.

Temos também umas palavras a dizer sobre bases aditivas. É fácil perceber que a métrica introduzida funciona como uma "lupa" que "olha" para o começo das

\footnotetext{
${ }^{3}$ Se $\gamma_{0}=0, \gamma_{1}=1$ e $\gamma_{n}=\gamma_{n-1}+\gamma_{n-2}$, então $\left\{\sqrt[n]{\gamma_{n}}\right\}_{n \geq 2}$ é estritamente crescente.
} 
bases aditivas, isto é, o "começo" das bases importa muito para o desenvolvimento deste trabalho. Como poderíamos modificar a métrica para obter uma "lupa" que "olhasse" para o infinito?

Uma maneira de fazer isso seria substituir a métrica $d$ pela métrica

$$
\begin{array}{rlc}
d_{\infty}: \mathcal{A} \times \mathcal{A} & \rightarrow & {[0, \infty)} \\
(A, B) & \mapsto\left|o_{\infty}(A)-o_{\infty}(B)\right|+\sum_{\alpha \in A \triangle B} \frac{1}{\alpha^{2}}
\end{array}
$$

Como é, portanto, o espaço métrico $\left(\mathcal{A}, d_{\infty}\right)$ ? Quais propriedades topológicas ele tem? É $(\mathcal{A}, d)$ homeomorfo a $\left(\mathcal{A}, d_{\infty}\right)$ ? Que tipo de teoremas aproximativos (como o teorema 3.3.15) podem ser obtidos?

A mesma ideia pode ser reproduzida para tentarmos estudar a conjectura 1.7.10 de Erdös-Turán: que alterações na métrica poderíamos inserir de modo que, fixada $A \in \mathcal{A}$, a sequência $\{r(n, A)\}_{n \in \mathbb{N}}$ nos desse informação relevante sobre esta conjectura?

Mais ainda, o que poderíamos falar se particionássemos $\mathcal{A}$ por meio da relação de equivalência

$$
A \text { está relacionada com } B \text { se, e somente se, } A(n) \sim B(n) \text { ? }
$$

Qual método aproximativo pode ser utilizado para estudar uma classe de equivalência desta relação de equivalência?

Por fim, poderíamos substituir a métrica $d$ por uma topologia $\tau$ em $\mathcal{A}$ tal que o espaço topológico $(\mathcal{A}, \tau)$ não é metrizável, mas ainda assim fornece informações relevantes sobre o conjunto das bases aditivas, ou sobre alguma base aditiva específica. Neste caso, qual uma boa maneira de definir $\tau$ ?

Estas perguntas, tanto as algébricas sobre a conjectura de Firoozbakht quanto as analíticas/topológicas relativas às bases aditivas, mostram que o trabalho ainda não acabou, e que há muito para se descobrir sobre estes dois fascinantes assuntos. 


\section{Referências Bibliográficas}

[1] http://www.primepuzzles.net/thepuzzlers/Firoozbakht.htm. Último acesso em 20 de junho de 2016.

[2] https://en.wikipedia.org/wiki/Legendre's_conjecture. Último acesso em 20 de junho de 2016.

[3] https://en.wikipedia.org/wiki/Brocard's_conjecture. Último acesso em 20 de junho de 2016.

[4] https://oeis.org/A182514. Último acesso em 20 de junho de 2016.

[5] P. A. Martin. Grupos, Corpos e Teoria de Galois. Textos Universitários do IME, Volume 2. Editora Livraria da Física. 429 p. 2010.

[6] J. K. Andersen. Primes in Arithmetic Progression Records. Disponível em: http://primerecords.dk/aprecords.htm. Último acesso em 25 de setembro de 2016.

[7] M. Aigner; G. M. Ziegler. As provas estão n'O LIVRO. Editora Edgard Blücher Ltda. $2^{\mathrm{a}}$ edição, 2002.

[8] D. Andrica. Note on a Conjecture in Prime Number Theory. Studia Univ. Babes-Bolyai Math. (1986) 31 (4): 44-48.

[9] A. Arhangel'skii; M. Tkachenko. Topological Groups and Related Structures. Atlantis Press / World Scientific, 2008. 800 p. (Atlantis Studies in Mathematics, Volume 1)

[10] E. Artin; P. Scherk. On the sum of two sets of integers. Annals of Mathematics, Second Series, Vol. 44, No. 2 (Apr., 1943), p. 138-142.

[11] C. Axler. New bounds for the prime counting function $\pi(x)$. Disponível em http://arxiv.org/pdf/1409.1780v7. Último acesso em 20 de junho de 2016 .

[12] R. C. Baker; G. Harmar; J. Pintz. The difference between consecutive primes, II. Proceedings of the London Mathematical Society (2001) 83 (3): 532-562.

[13] R. Balasubramanian; J.-M. Deshouillers; F. Dress. Problème de Waring pour les bicarrés, 1: schéma de la solution. C. R. Acad. Sci. Paris Sér. I 303 (1986), p. 85-88. 
[14] R. Balasubramanian; J.-M. Deshouillers; F. Dress. Problème de Waring pour les bicarrés, 2: résultats auxiliaires pour le théorème asymptotique. C. R. Acad. Sci. Paris Sér. I 303 (1986), p. 161-163.

[15] E. Catalan. Note extraite d'une lettre adressée à l'éditeur par Mr. E. Catalan, Répétiteur à l'école polytechnique de Paris. Journal für die reine und angewandte Mathematik. Volume 1844, Issue 27, Pages 192-192.

[16] H. H. Chan. Analytic Number Theory for Undergraduates. Monographs in Number Theory, Volume 3. World Scientific, 2009.

[17] K. Conrad. Expository papers in Algebraic number theory. (notas) Disponível

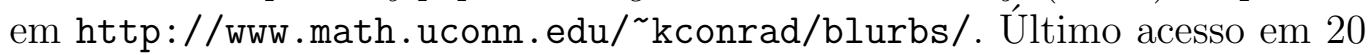
de junho de 2016 .

[18] H. Cramér. Some theorems concerning prime numbers. Ark. Mat. Astron. Fys. (1920), Volume 15, No. 5.

[19] H. Cramér. On the order of magnitude of the difference between consecutive prime numbers. Acta Arithmetica (1936), Volume 2, Issue 1, p. 23-46.

[20] H. Davenport. On Waring's problem for fourth powers. Annals of Mathematics, Second Series, Vol. 40, No. 4 (Oct., 1939), p. 731-747.

[21] J.-M. Deshouillers. Sur la majoration des sommes de Weyl biquadratiques. Ann. Scuola Norm. Sup. Pisa Cl. Sci. (4) 19 (1992), p. 291-304.

[22] J.-M. Deshouillers; F. Dress. Sommes de diviseurs et structure multiplicative des entiers. Acta Arithmetica (1988), Volume 49, Issue 4, p. 341-375.

[23] J.-M. Deshouillers; F. Dress. Sum of 19 biquadrates: on the representation of large integers. Ann. Scuola Norm. Sup. Pisa Cl. Sci. (4) 19 (1992), p. 113-153.

[24] J.-M. Deshouillers; F. Dress. Numerical results for the sums of five and seven biquadrates and consequences for sums of 19 biquadrates. Mathematics of Computation, Volume 61, Number 203, July 1993, p. 195 - 207.

[25] O. Endler. Teoria dos Números Algébricos. Projeto Euclides. IMPA, 1986.

[26] P. Erdös. On the arithmetical density of the sum of two sequences one of which forms a basis for the integers. Acta Arithmetica (1935), Volume 1, Issue 2, p. $197-200$.

[27] P. Erdös. Problems in number theory and combinatorics. Proc. 6th Manitoba conference on numerical math., 1976, p. 35-38.

[28] P. Erdös; P. Turán. On a problem of Sidon in additive number theory, and on some related problems. J. London Math. Soc. (1941) s1-16 (4), p. 212-215.

[29] H. Furstenberg. Ergodic behavior of diagonal measures and a theorem of Szemerédi on arithmetic progressions. Journal d'Analyse Mathématique (1977), Volume 31, Issue 1, p. 204-256. 
[30] C. Goldbach. [Carta] 7 de junho de 1742. Moscou [para] L. Euler, Berlim. 5f. Continuation sur les mêmes sujets. Deux théorèmes d'analyse. Disponível em: http://www.math.dartmouth. edu/ euler/correspondence/letters/000765.pdf. Último acesso em 03 de junho de 2016.

[31] W. T. Gowers. A new proof of Szemerédi's theorem. Geometric \& Functional Analysis GAFA, August 2001, Volume 11, Issue 3, p. 465-588.

[32] A. Granville. Harald Cramér and the distribution of prime numbers. Scandinavian Actuarial Journal (1995), Vol. 1, p. 12-28.

[33] B. Green; T. Tao. The primes contain arbitrarily long arithmetic progressions. Annals of Mathematics, Vol. 167, Issue 2 (2008), p. 481-547.

[34] H. A. Helfgott. Minor arcs for Goldbach's problem. Disponível em: http://arxiv.org/abs/1205.5252. Último acesso em 03 de junho de 2016.

[35] H. A. Helfgott. Major arcs for Goldbach's problem. Disponível em: http://arxiv.org/abs/1305.2897. Último acesso em 03 de junho de 2016.

[36] H. A. Helfgott. The ternary Goldbach conjecture is true. Disponível em: http://arxiv.org/abs/1312.7748. Último acesso em 03 de junho de 2016.

[37] H. A. Helfgott. The ternary Goldbach problem. Disponível em: http://arxiv.org/abs/1501.05438. Último acesso em 03 de junho de 2016 .

[38] Q.-H. Hou; Z.-W. Sun; H. Wen. On monotonicity of some combinatorial sequences. Disponível em: http://arxiv.org/abs/1208.3903v7. Último acesso em 03 de julho de 2016.

[39] G. G. Janusz. Algebraic Number Fields. (second edition) Graduate Studies in Mathematics, Volume 7. American Mathematical Society, 2005.

[40] A. Kourbatov. Verification of the Firoozbakht Conjecture for Primes up to Four Quintillion. Disponível em: http://arxiv.org/abs/1503.01744. Último acesso em 20 de junho de 2016.

[41] A. Kourbatov. Upper Bounds for Prime Gaps Related to Firoozbakht's Conjecture. Disponível em: http://arxiv.org/pdf/1506.03042. Último acesso em 20 de junho de 2016.

[42] A. Khinchin. Über ein metrisches problem der additiven zahlentheorie. Mat. Sb. (1933), Volume 40, Number 2, p. 180-189.

[43] A. Y. Khinchin. Three pearls of number theory. 1. ed. Dover Publications, 2010. 64 p. (Dover Books on Mathematics).

[44] J. M. Kubina; M. C. Wunderlich. Extending Waring's conjecture to 471600000. Mathematics of Computation, Volume 55, Number 192, October 1990, p. 815-820.

[45] S. Lang. Algebra. Revised Third Edition. Springer-Verlag, 2002. 914 p. (Garduate Texts in Mathematics 211). 
[46] E. L. Lima. Espaços métricos. Quarta edição, segunda impressão. IMPA, 2007. 299 p. Projeto Euclides.

[47] U. V. Linnik. On Erdös's theorem on the addition of numerical sequences. Rec. Math. [Mat. Sbornik] N.S. (1942), Volume 10(52), Number 1-2, p. 6778.

[48] M. B. Nathanson. Additive number theory: the classical bases. 1. ed. SpringerVerlag, 1996. 344 p. (Graduate Texts in Mathematics 164).

[49] M. B. Nathanson. Additive number theory: inverse problems and the geometry of sumsets. 1. ed. Springer-Verlag, 1996. 296 p. (Graduate Texts in Mathematics 165).

[50] M. B. Nathanson. Elementary methods in number theory. 1. ed. SpringerVerlag, 2000. 514 p. (Graduate Texts in Mathematics 195).

[51] M. B. Nathanson. Cassels bases. Disponível em: http://arxiv.org/abs/0905.3144. Último acesso em 03 de junho de 2016 .

[52] K. Mahler. On the fractional parts of the powers of a rational number, II. Mathematika (1957), Volume 4, Issue 02, p. 122-124.

[53] H. Maier. Primes in short intervals. Michigan Math. J., Volume 32, Issue 2 (1985), p. 221-225.

[54] H. B. Mann. A proof of the fundamental theorem on the density of sums of set of positive integers. Annals of Mathematics, Second Series, Vol. 43, No. 3 (Jul., 1942), p. 523-527.

[55] D. Marques. Teoria dos Números Transcendentes. Coleção Textos Universitários. Editora SBM. 223 p. 2013.

[56] D. A. Markus. Number Fields. Universitext. Springer-Verlag, 1977.

[57] P. Mihailescu. Primary cyclotomic units and a proof of Catalans conjecture. Journal für die reine und angewandte Mathematik. Volume 2004, Issue 572, Pages 167-195.

[58] J. S. Milne. Algebraic Number Theory. (notas) Disponível em http://www.jmilne.org/math/CourseNotes/ANT.pdf. Último acesso em 20 de junho de 2016.

[59] R. A. Mollin. Algebraic Number Theory. (second edition) Discrete Mathematics and its applications. Chapman and Hall/CRC, 2011.

[60] J. R. Munkres. Topology. Second Edition. Pearson, 2000. 537 p.

[61] M. R. Murty; J. Esmonde. Problems in Algebraic Number Theory. (second edition) Graduate Texts in Mathematics 190. Springer, 2005. 
[62] L. Oppermann. Om vor Kundskab om Primtallenes Mangde mellem givne Grcendser. Oversigt over det Kongelige Danske Videnskabernes Selskabs Forhandlinger og dets Medlemmers Arbejder (1882): 169-179.

[63] P. Pollack. Not always buried deep: a second course in elementary number theory. 1. ed. American Mathematical Society, 2009. 303 p.

[64] F. Pollock. On the extension of the principle of Fermat's theorem of the polygonal numbers to the higher orders of series whose ultimate differences are constant. With a new theorem proposed, applicable to all the orders. Abstracts of the Papers Communicated to the Royal Society of London, Vol. 5 (1843-1850), p. 922-924.

[65] D.H.J. Polymath. Variants of the Selberg sieve, and bounded intervals containing many primes. Research in the Mathematical Sciences 1:12, 2014.

[66] P. Ribenboim. Classical Theory of Algebraic Numbers. Universitex. Springer, 2001.

[67] P. Ribenboim. Números Primos: Mistérios e Recordes. Editora LTC. Coleção Matemática Universitária. 2001.

[68] N. P. Romanoff. Über einige Sätze der additiven Zahlentheorie. Mathematische Annalen, December 1934, Volume 109, Issue 1, p. 668-678.

[69] B. Rosser. The $n$-th Prime is Greater than $n \log (n)$. Proceedings of the London Mathematical Society (1939) s2-45 (1): 1-20.

[70] J. Rotman. Galois Theory. (second edition) Springer. Universitext. 157 p. 2013.

[71] I. Z. Ruzsa. Essential components. Proc. London Math. Soc. (1987) s3-54 (1), p. $38-56$.

[72] P. Samuel. Algebraic Theory of Numbers. Dover, 2008.

[73] U. V. Satyanarayana. On the representation of numbers as sums of triangular numbers. The Mathematical Gazette, Vol. 45, No. 351 (Feb., 1961), p. 40-43.

[74] R. Schoof. Catalan's Conjecture. Universitext, Springer-Verlag, 2008.

[75] I. Stewart. Galois Theory. (thrid edition) Chapman and Hall/CRC. 328 p. 2003

[76] I. Stewart; D. Tall. Algebraic Number Theory and Fermat's Last Theorem. (third edition) A K Peters, Ltd, 2002.

[77] A. Stöhr. Gelöste und ungelöste Fragen über Basen der natürlichen Zahlenreihe. I, II. Journal für die reine und angewandte Mathematik (1955), Volume 194, p. 40-65, 111-140.

[78] S. Shokranian; M. Soares; H. Godinho. Teoria dos números. Brasília: Editora Universidade de Brasília, $2^{a}$ edição, 1999. 325 p. 
[79] T. Oliveira e Silva; S. Herzog; S. Pardi. Empirical verification of the even Goldbach conjecture and computation of prime gaps up to $4 \cdot 10^{18}$. Mathematics of Computation, Volume 83, Number 288, July 2014, p. 2033-2060. Article eletronically published on November 18, 2013.

[80] J. Singer. A theorem in finite projective geometry and some applications to number theory. Trans. Amer. Math. Soc. 43 (1938), 377-385.

[81] E. Szemerédi. On sets of integers containing no $k$ elements in arithmetic progression. Acta Arithmetica (1975), Volume 27, Issue 1, p. 199-245.

[82] Y. Wang; B.X. Zhu. Proofs of some conjectures on monotonicity of numbertheoretic and combinatorial sequences. Science China Mathematics. November 2014, Volume 57, Issue 11, p. 2429-2435.

[83] L. C. Washington. Introduction to Cyclotomic Fields. 2. ed. Springer-Verlag, 1997. 490 p. (Graduate Texts in Mathematics 83).

[84] G. L. Watson. Sums of eight values of a cubic polynomial. J. London Math. Soc. (1952) s1-27 (2), p. 217-224.

[85] S. Weintraub. Galois Theory. Second Edition. Springer-Verlag. Universitext, 2009. 212 p.

[86] S. Willard. General Topology. Addison-Wesley Publishing Company. 1970.

[87] Y. Zhang. Bounded gaps between primes. Annals of Mathematics, Vol. 179, No. 3, p. 1121-1174, 2014. 